\title{
Utilizing early cellular changes to explore biological responses to individual chemical and mixture exposures
}

Julie Anne Vrana

Follow this and additional works at: https://researchrepository.wvu.edu/etd

\section{Recommended Citation}

Vrana, Julie Anne, "Utilizing early cellular changes to explore biological responses to individual chemical and mixture exposures" (2015). Graduate Theses, Dissertations, and Problem Reports. 6880.

https://researchrepository.wvu.edu/etd/6880

This Dissertation is protected by copyright and/or related rights. It has been brought to you by the The Research Repository @ WVU with permission from the rights-holder(s). You are free to use this Dissertation in any way that is permitted by the copyright and related rights legislation that applies to your use. For other uses you must obtain permission from the rights-holder(s) directly, unless additional rights are indicated by a Creative Commons license in the record and/ or on the work itself. This Dissertation has been accepted for inclusion in WVU Graduate Theses, Dissertations, and Problem Reports collection by an authorized administrator of The Research Repository @ WVU.

For more information, please contact researchrepository@mail.wvu.edu. 
Utilizing early cellular changes to explore biological responses to individual chemical and mixture exposures

by

Julie Anne Vrana

Dissertation submitted to the Eberly College of Arts and Sciences

at West Virginia University

in partial fulfillment of the requirements

for the degree of

Doctor of Philosophy

in

Chemistry

\author{
Approved by \\ Jonathan Boyd, Ph.D., Committee Chairperson \\ Fred King, Ph.D. \\ Justin Legleiter, Ph.D. \\ James O'Callaghan, Ph.D. \\ Brian Popp, Ph.D. \\ C. Eugene Bennett Department of Chemistry \\ Morgantown, West Virginia \\ 2015
}

Keywords: Toxicity, chemical exposure, mixtures, prediction, signal transduction, in vitro

Copyright 2015 Julie Anne Vrana 


\begin{abstract}
Utilizing early cellular changes to explore biological responses to individual chemical and mixture exposures
\end{abstract}

by

Julie Anne Vrana

Humans are continuously exposed to a vast number of chemicals, whether it be from the air we breathe, the water we drink, or the medications we take daily. Early cellular changes after exposure to chemical insult, both individual chemicals and mixtures (two or more chemicals) thereof, can offer a wealth of information about cellular adaptation (e.g., cell death or survival decision processes). From this understanding, better prediction models for chemical risk assessment, such as toxicity or carcinogenicity, can be elucidated. Further, these prediction models can greatly improve the large backlog of chemicals waiting to be evaluated for potential adverse effects. One approach to understand cellular changes and responses after chemical or mixture exposure is with toxicodynamics. From a toxicodynamic approach, a host of information can be determined, such as spatiotemporal interactions of chemical insult with biological targets, the corresponding disruption of intracellular pathways and bioenergetics, and downstream effects after exposure. Appropriately measuring these dynamic cellular changes is imperative. The recent advances in molecular biology, high-throughput in vitro screening assays, and numerous computational techniques have allowed toxicologists to collect large data sets on signaling pathways that are perturbed in response to chemical insults. From these early cellular perturbations, whether they be signaling proteins, biomolecules (e.g., ATP, hormones, NADH), or ions (e.g., $\mathrm{Ca}^{2+}$ or $\mathrm{K}^{+}$), in response to a wide range of doses, especially low concentrations, improved risk assessment prediction models for individual chemical and mixture exposures can be utilized by many fields, such as risk assessment for environmental toxicology and target molecule/pathway analysis for drug development and pharmacology. 


\section{DEDICATION}

This dissertation is dedicated to the memory of my extraordinary grandparents, Paul and Julia Ondrasik and Charles and Rosalie Vrana, and my remarkable cousin, Ashley Baker. Each of these individuals inspired me through their tireless work ethic,

appreciation of life and little moments, wisdom, sense of humor, and endless love of family and friends. 


\section{ACKNOWLEDGEMENTS}

First and foremost, I am extremely grateful and appreciative for my advisor, Dr. Jonathan Boyd. His support, wisdom, patience, and trust in me over the past 5 years have allowed me to become the researcher and person I am today. The opportunities afforded to me, such as grant writing, manuscript preparation, research project development, and scientific conference participation, have dramatically improved my scientific career. Thank you for taking a chance on a "diamond in the rough," and always pushing me to be better.

I would like to thank my committee members, Dr. Fred King, Dr. Justin Legleiter, Dr. James O’Callaghan, and Dr. Brian Popp, for their encouragement, guidance, support, patience, and support throughout my graduate career.

To my fellow lab mates, Dr. Holly Currie, Alice Han, and Maren Prediger, thank you for being a sounding board, supplying an endless amount of laughter and social support, as well as genuine camaraderie. Whether it be proofreading manuscripts, aliquoting blood and saliva samples at 1:00 AM for two months straight, going for ice cream breaks to Dairy Queen, being a shoulder to cry on through the loss of my grandparents and cousin, or our most sacred stress-relieving trips to Sports Page ("Spage"), I could not have gotten through graduate school without each of you.

To the outstanding and supportive undergraduates who have been a part of these various projects during my time at West Virginia University, Amber Cook, Nick Rubenstein, Stan Strawbridge, Emily Fabyanic, and Connie Mitchell, thank you for 
sacrificing your time to help with these diverse projects. This work would not be possible without each and every one of you.

I would like to thank my best friend, my rock, and my everything, Andrew Miller. We have been through this roller coaster ride together and I know it has not been easy. Driving 600 miles through the rain and snow round trip for one short weekend together each month has certainly been challenging but we made it work. Thank you for listening to me complain about graduate school, lifting me up when I thought I wasn't good enough, and always making me laugh. I am extremely excited and overjoyed for our next adventure together.

I would also like to thank my parents, Dan and Anne Vrana, and my brother Andy for their constant support and encouragement. There will never be appropriate words to describe my gratitude for your endless love and faith in me. Thank you for showing me what hard work and responsibility are and how I can always use my talents to help others. Also, thank you for listening to me complain about or just talk science, even though I am sure it is completely boring. You never allowed me to give up and always pushed me to get to the next stage in life. Finally, thank you for helping me take time for a break, whether it be Pirates games, watching TV, or having a margarita. I would not be the person I am today without you.

Finally, to all of my family and friends, thank you. This would never have been possible without your encouragement, love, and support. 


\section{TABLE OF CONTENTS}

Abstract $\quad$ ii

Dedication $\quad$ iii

Acknowledgements iv

Table of Contents vi

List of figures $\quad x$

List of tables $\quad$ xii

1. Introduction: Utilizing early cellular changes to explore biological 1 responses to individual and chemical mixtures

1.1 Classification of toxic agent and exposure effects - a toxicological 3

perspective

1.1.1 Dose-response for chemical exposure toxicity testing and 4 risk assessment

1.1.2 Chemical mixtures 5

1.1.3 Mode of action vs mechanism of action $\quad 7$

1.2 Early cellular changes post-exposure 9

1.2.1 Intracellular signaling perturbations associated with exposure $\quad 11$

1.2.2 Bioenergetic changes post-exposure $\quad 15$

$\begin{array}{ll}\text { 1.2.3 Timescale of exposure effects } & 18\end{array}$

1.3 Experimentally testing early cellular changes that may contribute to 19

exposure sensing and response

$\begin{array}{ll}\text { 1.3.1 In vitro cell culture } & 19\end{array}$

1.3.2 Real time in vitro assays to measure early cellular changes 22

1.3.2.1 Mitochondrial bioenergetics assays: NADH 22 generation and oxygen consumption

1.3.2.2 Plasma membrane degradation 25

1.3.3 Endpoint in vitro assays to measure early cellular changes $\quad 26$ and long term exposure effects 
2. Forecasting cell death dose-response to single chemicals in vitro 41

2.1 Introduction 43

2.2 Materials and Methods $\quad 46$

2.2.1 Materials $\quad 46$

$\begin{array}{ll}\text { 2.2.2 Cell culture } & 47\end{array}$

$\begin{array}{ll}\text { 2.2.3 Dosimetry } & 47\end{array}$

2.2.4 MTT assay $\quad 48$

2.2.5 Oxygen consumption assay 48

2.2.6 NADH assay 49

2.2.7 Cellular ATP assay 49

2.2.8 Multiplex phosphoprotein assay $\quad 50$

2.2.9 Plasma membrane degradation assay 51

2.2.10 Statistical analysis $\quad 52$

2.2.11 Signaling pathway analysis $\quad 52$

2.3 Results 53

2.3.1 HepG2 viability in response toTDZD-8 and MEK inh II 51

2.3.2 Estimation of ATP generation to determine critical 54 signaling events

2.3.3 Multiplex assay to determine phosphorylation of key proteins $\quad 58$

2.3.4 Hierarchical cluster analysis $\quad 61$

2.3.5 Forecasting dose-response from hierarchical cluster analysis $\quad 62$

2.4 Discussion $\quad 65$

2.5 References $\quad 71$

3. Forecasting cell death dose-response to mixtures in vitro 77

$\begin{array}{ll}\text { 3.1 Introduction } & 79\end{array}$ 
3.2 Materials and Methods $\quad 82$

3.2.1 Materials - Deguelin and KCN 82

3.2.2 Materials - MEK inh II and TDZD-8 82

3.2.3 Cell culture $\quad 83$

3.2.4 Dosimetry $\quad 83$

3.2.5 Multiplex phosphoprotein assay - Degulin and KCN mixtures $\quad 84$

3.2.6 Multiplex phosphoprotein assay - MEK inh II and 85 TDZD-8 mixtures

3.2.7 Plasma membrane degradation assay $\quad 86$

3.2.8 Statistical analysis $\quad 86$

$\begin{array}{ll}3.3 \text { Results } & 87\end{array}$

3.3.1 Using Bliss independence (response addition) to predict 87 relative phosphorylation during critical signaling events

3.3.2 Forecasting mixtures dose-response with hierarchical cluster 91 analysis

3.4 Discussion 94

$\begin{array}{ll}3.5 \text { References } & 97\end{array}$

Appendix 3.1 Observed relative phosphorylation of deguelin and KCN $\quad 100$ mixtures (400 min post-exposure)

Appendix 3.2 Observed relative phosphorylation of MEK inh II and 105 TDZD-8 mixtures (20 min post-exposure)

Appendix 3.3 Observed relative phosphorylation of MEK inh II and TDZD-8 mixtures (40 min post-exposure

\section{Using mixtures to ameliorate undesired side-effects of deguelin 117}

$\begin{array}{ll}4.1 \text { Introduction } & 119\end{array}$

$\begin{array}{ll}\text { 4.2 Materials and Methods } & 123\end{array}$

4.2.1 Materials 123

$\begin{array}{ll}\text { 4.2.2 Cell culture } & 123\end{array}$

$\begin{array}{ll}\text { 4.2.3 Dosimetry } & 124\end{array}$

$\begin{array}{ll}\text { 4.2.4 MTT assay } & 124\end{array}$ 
4.2.5 Oxygen consumption assay

4.2.6 Bio-plex multiplex immunoassay

4.2.7 Statistical analysis

4.3.1 Deguelin as a monotherapy

4.3.2 HepG2 viability (in response to deguelin) may not be directly associated with $\mathrm{O} 2$ consumption

4.3.3 Endogenous phosphorylation response to deguelin exposes a potential secondary therapeutic target to deguelin

4.3.5 Mixtures approach retains efficacy while decreasing deguelin's effect on $\mathrm{O} 2$ consumption

4.4 Discussion

4.5 References

\section{Future directions: Expanding concepts to human interaction}

5.1 Introduction

5.2 Materials and Methods

5.2.1 Subjects

5.2.2 Salivary biomarkers

5.3 Results and Discussion

5.4 Conclusion 


\section{LIST OF FIGURES}

1.1 Mechanism of action $\quad 8$

1.2 Early cellular changes related to biological outcome 11

1.3 Mechanism of phosphorylation 13

1.4 Mitochondria 16

1.5 Bead-based ELISA 28

2.1 HepG2 viability to determine relevant TDZD-8 and MEK inh II doses 54

2.2 Theoretical ATP generation and activity models indicate key bifurcation $\quad 57$ points for HepG2 cells exposed to TDZD-8 and MEK inh II

2.3 Protein phosphorylation responses of HepG2 cells to TDZD-8 60

2.4 Protein phosphorylation responses of HepG2 cells to MEK inh II 60

2.5 Forecasting plasma membrane degradation from two-way hierarchical 63 cluster analysis distances

2.6 Ingenuity pathway analysis of phosphoprotein responses 68

3.1 Relative phosphorylation responses of HepG2 cells exposed to deguelin or KCN 88

3.2 Relative phosphorylation responses of HepG2 cells exposed to MEK inh II or 89 TDZD-8

3.3 Deguelin and KCN mixture correlation $\quad 90$

3.4 MEK inh II and TDZD-8 mixture correlation (20 min) 90

3.5 MEK inh II and TDZD-8 mixture correlation (40 min) 90

3.6 Observed phosphoprotein responses for PM degradation forecast 91

3.7 Bliss independence-predicted phosphoprotein responses for PM 91 degradation forecast

3.8 Relative PM degradation (predicted and observed) of HepG2 cells at $400 \mathrm{~min} \quad 92$ post-exposure to deguelin and $\mathrm{KCN}$

3.9 Relative PM degradation (predicted and observed) of HepG2 cells at 20 and 93 40 min post-exposure to MEK inh II and TDZD-8

4.1 Percent viability of HepG2 cells in response to 24 hour exposure of $0.01-100 \mu \mathrm{M}$ deguelin

4.2 Percent oxygen consumption of HepG2 cells in response to $0.01-100 \mu \mathrm{M}$ deguelin 
4.3 Relative phosphorylation of protein targets in response to $0.01-100 \mu \mathrm{M}$ deguelin

4.4 Relative p38MAPK phosphorylation in response to deguelin, SB202190, and mixtures of deguelin with SB202190

4.5 Percent viability of HepG2 cells in response to 24 hour exposure of deguelin alone and in combination with SB202190

4.6 Percent oxygen consumption of HepG2 cells in response to deguelin alone, and in combination with SB202190

5.1 Initial salivary cytokine responses as an individual

5.2 Initial salivary cytokine responses as a team 


\section{LIST OF TABLES}

5.1 Time to complete first mission as an individual and team

5.2 Mean summed cytokine responses as an individual and team 
Chapter 1

Introduction to early cellular changes that may contribute to individual and mixture responses after exposure 


\section{Introduction: Utilizing early cellular changes to explore biological responses to individual and chemical mixtures}

The early cellular changes initiated by external stimuli (whether that be chemical, physical, or biological agents) can offer a host of information about the adaptive response and adverse effects related to individual chemical and mixture exposures. Notable examples of early cellular changes are perturbations of dynamic intracellular signaling networks and alterations in cellular bioenergetics, such as increased/decreased oxygen consumption or electron transport chain uncoupling. Due to the interconnectivity of various effector proteins and biomolecules, activity at a distinct intracellular location can have consequences at distal locations. Additionally, as the primary tenet of toxicology proclaims, "it is the dose that makes the poison," a better understanding of cellular changes in response to a range of concentrations, especially low dose exposures, is essential to toxicity testing and chemical risk assessment. Finally, the ability to measure the rapid and dynamic cellular responses to exposure is critical for an enhanced understanding of toxicity. The experimental conditions and assays capable of capturing relevant mechanistic information for toxicity testing will be discussed in this chapter. A better understanding of the mechanistic components related to chemical response has

numerous implications across many fields, such as risk assessment for toxicology and target molecule/pathway analysis for drug development and pharmacology. With mechanistic data collected from various doses, intracellular proteins and biomolecules, and cellular endpoints, better toxicity prediction models can be developed for individual chemical and mixtures risk assessment. 


\subsection{Classification of toxic agent and exposure effects - a toxicological perspective}

Humans are continuously exposed to a plethora of chemicals daily. These chemicals can be traced to a variety of sources, such as environmental, pharmaceutical, or industrial. With the considerable amount of individual chemicals humans can be exposed to on a daily basis, this begs the question, what classifies a chemical or agent as "toxic"? A toxic agent, whether it be chemical, biological, or physical, can be classified by their use (e.g., pharmaceutical, pesticide, additive, etc.), source (e.g., human-made, plant or animal toxin, etc.), target organ(s) (e.g., brain, liver, heart, etc.), and effects (e.g., cardiotoxicity, carcinogenicity, immunogenicity, etc.) (Casarett and Klaassen, 2008). A primary tenet of toxicology states that all chemical agents are toxic, but it is the dose that determines if an agent is toxic or safe (Pottenger and Gollapudi, 2009). The classification of what constitutes an agent as toxic, and the threshold thereof, can be ambiguous. However, the experimental characterization of an agent that incorporates chemical properties and biological exposure effects is useful for toxicological risk assessment. The work described in this dissertation will primarily focus on exogenous chemical stressors, also known as xenobiotics.

Appropriately characterizing biological effects of a single agent exposure can be a daunting task. Toxicological risk assessment determines the quantitative estimate of possible effects of a xenobiotic on human health (Casarett and Klaassen, 2008). Following exposure, xenobiotic effects can be adverse, deleterious, or dangerous to an organism (Edwards and Aronson, 2000). Conversely, some effects can be beneficial, such as pharmaceutical side effects of antihistamines (drowsiness) or oral contraceptives (decrease acne severity). To properly address the adverse or beneficial nature of a 
chemical exposure, the dose and time course of toxicity/adaptation needs to be elucidated.

\subsubsection{Dose-response for chemical exposure toxicity testing and risk assessment}

One of the most central concepts utilized for toxicity studies and risk assessment is the dose-response relationship (Calabrese and Baldwin, 2003b). The response variable can be any desired effect to be measured, such as cell death, survival, or cellular oxygen consumption, and the independent variable would be the doses required to elicit a response. There are many types of dose-response models used in toxicity testing, but the most dominant is the threshold model (Calabrese and Baldwin, 2003a). The threshold model has been used in many scientific disciplines, such as biology, pharmacology, and toxicology, and has been the primary model for regulatory agencies, such as the U.S. Federal Drug Administration (FDA) and Environmental Protection Agency (EPA) (Calabrese and Baldwin, 2003a). In the threshold model, depending upon the effect measured and assay sensitivity, there exists a dose below which the probability of a measured response for a sample or individual compared to control is zero (Cox, 1987). This threshold dose is also referred to as the no observed adverse effect level (NOAEL) (Allen et al., 1994). An alternative model, the hormesis model, has also proven to be useful for low-dose (below the NOAEL) risk assessment (Calabrese, 2009) and has seen a recent surge in interest due to advances in molecular toxicology testing (Calabrese and Baldwin, 2003b). The hormesis model is a biphasic dose-response relationship and can be 
succinctly described as low dose activation followed by high dose inhibition, which can appear as a U-shaped or J-shaped dose-response curve (Calabrese, 2008).

The renewed interest in hormesis as a valid model for toxicity testing has opened the door for low-dose biochemical and molecular toxicology research (Calabrese, 2008). It is important to note that the hormetic dose-response should also consider time in toxicity testing. This is due to the fact that hormesis responses may be a compensatory response that follows the initial disruption in homeostasis, resulting in the characteristic low-dose stimulatory response (Calabrese, 2001). Biological systems are highly coordinated and dynamic (Kholodenko et al., 2010), the exclusion of temporal response in risk assessment modeling would ignore the ability of an organism to adapt and respond to a low-dose exposure. Therefore, toxicological risk assessment needs to be inclusive of the spatiotemporal aspect of biological response post-exposure as well as a wide range of doses, including low doses.

\subsubsection{Chemical mixtures}

Current chemical exposure risk assessment is primarily carried out for single xenobiotics (Cedergreen et al., 2008), however, in reality humans are continuously exposed to a vast number of components, whether they be chemical, physical, or biological agents, at various doses, and through a variety of exposure routes on a daily basis (Groten et al., 2001). Understanding and ultimately predicting the possible combined effects of a given mixture exposure is necessary for risk assessment toxicology. There are two types of mixtures: simple and complex. Simple mixtures 
contain a small number of different chemicals and the composition is known. Complex mixtures contain hundreds to tens of thousands individual chemicals, of which the composition (dose or constituents) is not known (Feron et al., 1998). Experimentally determining the effects of all possible mixture combinations, or even binary mixtures, for a range of doses at different time points is not physically or financially possible (Cedergreen et al., 2008). Therefore, adequate models capable of predicting mixture responses are necessary for various sectors, such as pharmacological adverse interaction risk assessments and environmental exposure risk assessments. The two most commonly used and accepted mixture prediction models are Loewe additivity (dose addition) and Bliss independence (response addition) (McCarty and Borgert, 2006).

Dose addition is typically used when two or more chemical agents have a similar mechanism of action (Borgert et al., 2004). Dose addition is based on the theory that two chemicals in a mixture act as a dilution of each other (Loewe and Muischnek, 1926; Berenbaum, 1989). Traditional dose additivity can be described as shown in Equation 1:

$$
\sum \frac{\left[\alpha^{\prime}\right]_{i}}{E[\alpha]_{i}}=1
$$

where $\alpha^{\prime}$ is the dose of the chemical $i$ when administered as a mixture producing response $E$, and $\alpha$ is the concentration of the chemical agent, $i$, required to produce the response effect $E$ when administered alone (single exposure). Equation 1 can be used for $n$ number of agents as a mixture. If the overall expression is $<1$, the mixture is considered synergistic. Conversely, if the overall expression is $>1$, the mixture is considered antagonistic (Rajapakse et al., 2001). 
Response addition (Bliss independence) is typically used for two (or more) chemicals that do not have the same mechanism of action, that is, the organism will respond to each chemical agent independently, as though the other agent(s) are not present (Bliss, 1939; McCarty and Borgert, 2006). The prediction of mixture effects using response addition is equivalent to the conditional sum of independent chemical effect probabilities (Boyd et al., 2011). Response addition can be described by the expression (equation 2)

$$
f\left(\alpha_{m i x}\right)=1-\prod\left[1-F\left(\alpha_{i}\right)\right]
$$

where $F\left(\alpha_{i}\right)$ is the response effect produced by chemical $i$ at dose $\alpha$. Since this is a probabilistic model, $F\left(\alpha_{i}\right)$ cannot be greater than 1. Dose addition and response addition models are generalizations of nonlinear regression models, such as a Gompertz growth curve or Hill plot, and can be easily compared to observed mixture dose-response curves (Rajapakse et al., 2001; Boyd et al., 2011).

\subsubsection{Mode of action vs mechanism of action}

Regulatory guidelines for mixture risk assessments rely heavily on the similarity (or dissimilarity) of two chemical components' mechanistic information for model selection of a given mixture (USEPA, 1986; USEPA, 1999; USEPA, 2000; ATSDR, 2001b; ATSDR, 2001a; USEPA, 2002). In this discussion of prediction models for mixture toxicity, the term "mechanism of action" is used loosely. The term "mechanism" of action is often used interchangeably with "mode" of action (Borgert et al., 2004). However, these terms have specific definitions, but are often defined differently 
depending upon the literature article cited (Aptula and Roberts, 2006; Spurgeon et al., 2010). Traditionally, "mechanism of action" refers to the series of molecular events from the absorption of an effective dose of an agent to the eventual biological response (Butterworth et al., 1995). To fully describe the mechanism of action for a chemical, the components outlined in Figure 1.1 would need to be determined experimentally.

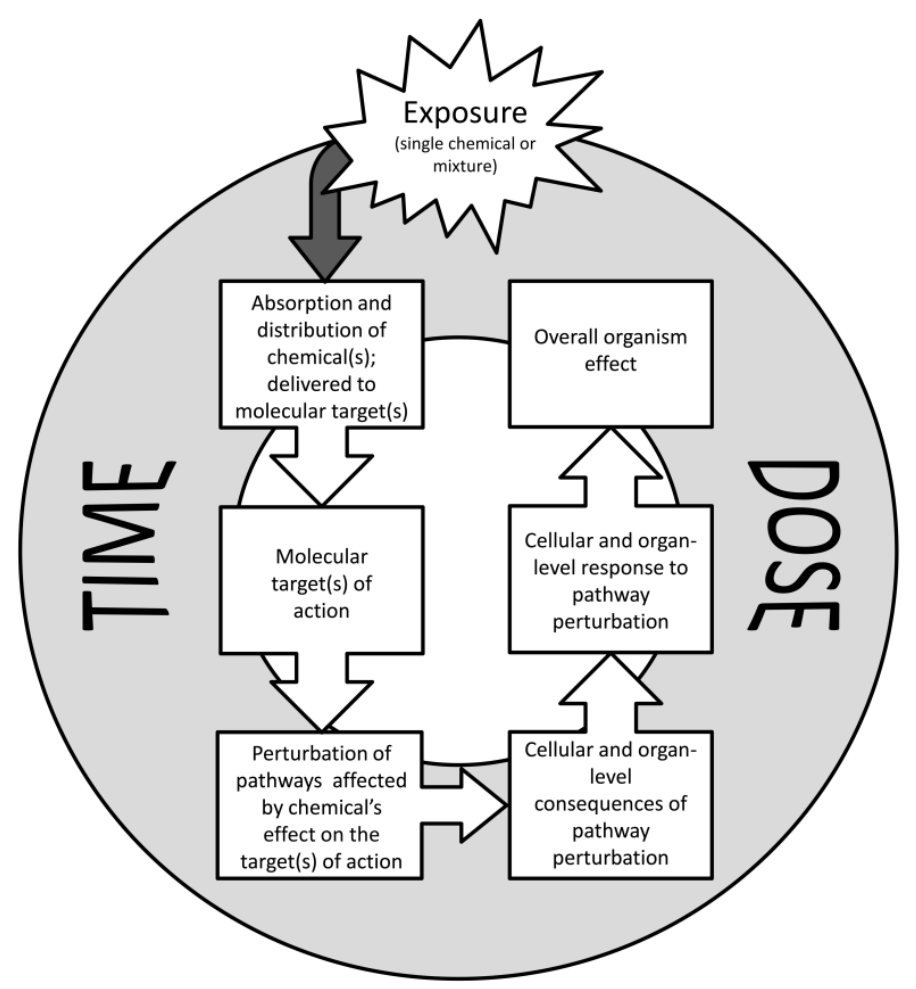

Figure 1.1. Mechanism of action. Schematic outlining the components necessary to elucidate the mechanism of action for an exposure. All steps must include the temporal and dose components to fully understand mechanism of action for a given chemical or mixture exposure.

The term "mode of action" is a more generalized way to describe a chemical's action on a given organism (Schlosser and Bogdanffy, 1999). The term "mode of action" describes the type of observed response of an organism exposed to a given chemical or mixture and may only refer to the significant events or components of the mechanism 
necessary for producing a particular biological response or toxic effect (Dellarco and Wiltse, 1998). Regardless of the terminology, the suitability of either model for a given experiment or effect prediction remains debatable when thorough mechanisms or modes of action are unknown, especially for low dose studies (Jonker et al., 2005; Spurgeon et al., 2010). Often, the models fail to predict mixtures toxicity when an observed mixture acts synergistically (Meled et al., 1998; Forget et al., 1999), antagonistically (Posthuma et al., 1997; Van Gestel and Hensbergen, 1997), or dose-dependent subtle interactions (e.g., synergy for low dose mixtures and antagonist for high dose mixtures) (Gennings et al., 2002; Jonker et al., 2004). To address these challenges, future model development should incorporate low dose (i.e., below the NOAEL) mixtures mechanistic effects on the appropriate spatiotemporal scale.

\subsection{Early cellular changes post-exposure}

Understanding and ultimately predicting potential biological effects and health outcomes from single chemical or mixture exposure remains an arduous task for risk assessment. The predominant approach for toxicity testing relies heavily on whole animal studies evaluating observable apical responses, such as clinical effects or pathologic changes from high dose exposures (Spurgeon et al., 2010). While many of these studies have been thorough, the amount of time and resources required to carry out this lowthroughput methodology has left this approach unable to meet the demands of current toxicology needs (NRC, 2007). The enormous backlog of chemicals waiting to be evaluated for toxic outcome has inspired a paradigm shift in toxicity testing, proposed by 
the U.S. National Research Council (NRC) report, Toxicity Testing in the 21st Century: A Vision and a Strategy (NRC, 2007; Bhattacharya et al., 2011). The NRC proposed a transition from in vivo low-throughput animal toxicity testing to an in vitro highthroughput approach utilizing well-designed mechanistic information-based assays. This in vitro approach would take advantage of early cellular perturbations post-exposure associated with toxicity endpoints in human cell lines and tissues to elucidate mechanistic information regarding the mode(s) of action for a potential xenobiotic or mixture (AtteneRamos et al., 2013). Additionally, the high-throughput nature of this approach would allow for testing on a wide range of doses, especially low doses, which is not currently possible with traditional whole animal studies (Bhattacharya et al., 2011). New risk assessment approaches would include a suite of assays in order to cast a wide net on early cellular changes, such as changes in cellular bioenergetics, and various pathway perturbations, such as alterations in post-translational modifications post-exposure, to fully understand the cellular response, whether that be adaptation after exposure to a new homeostatic state, or cell death (Andersen and Krewski, 2009).

Recent advances in systems toxicology have opened the door for the collection and analysis of large amounts of mechanistic data across a wide dosing range and time scale. Incorporating toxicodynamic factors for risk assessment can aid in our understanding of xenobiotic toxicity. The term toxicodynamics can be succinctly described as what the toxicant does to the body. Toxicodynamic analyses interrogate the spatiotemporal interaction of a xenobiotic with biological targets, the corresponding disruption of molecular pathways and bioenergetics, and downstream effects after exposure (Boelsterli, 2007). 
Marrying toxicokinetic and toxicodynamic approaches for risk assessment can offer a wealth of knowledge regarding xenobiotic exposure and the biological

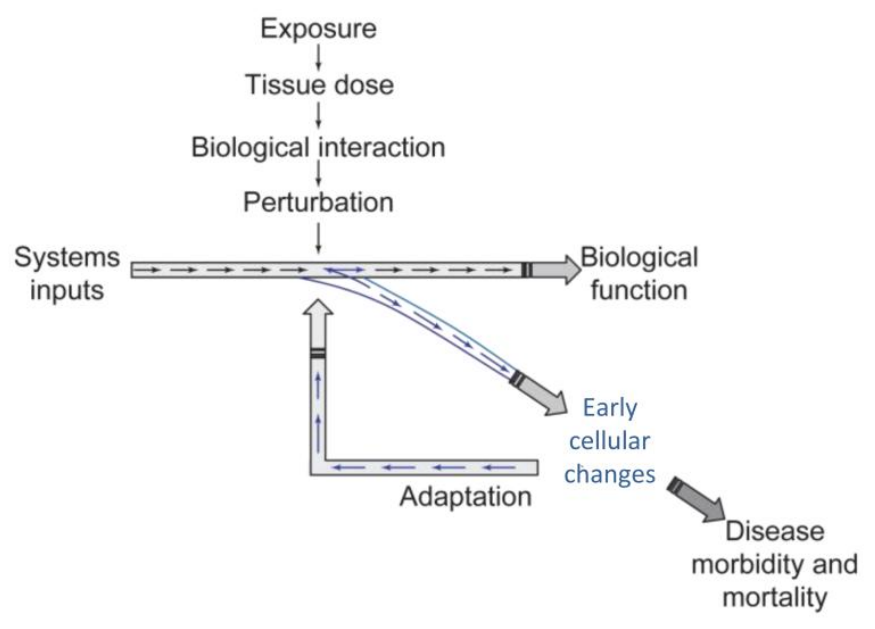

Figure 1.2. Early cellular changes related to biological outcome. Toxicokinetic components of exposure involve the absorption of the chemical exposure, biotransformation, and distribution resulting in the tissue dose. The chemical then interacts with molecular targets, perturbing endogenous pathways and processes, resulting in early cellular changes, which contributes to the toxicodynamic response post-exposure. Early cellular changes can lead to adverse effects (toxicity) or adaptive response and survival. Adapted from Andersen et al. (2005). Reprinted with permission from Elsevier.

perturbations associated with exposure and their corresponding effects (Figure 1.2). Perturbations of biological processes by xenobiotic exposure can elicit early cellular changes, leading to an adaptive stress response for continued survival or adverse response leading to toxicity (Andersen, 2010). Early cellular changes corresponding to toxicodynamics will be highlighted in this work, specifically intracellular signaling perturbations (e.g., post-translational modifications after chemical exposure) and alterations in cellular bioenergetics.

\subsubsection{Intracellular signaling perturbations associated with exposure}

The toxicodynamic components of early cellular changes, biological perturbations, and corresponding response yield valuable mechanistic information for toxicity risk assessment. Early and late effects associated with chemical exposure are 
mediated by plasma membrane receptor proteins acting as a sensor and their downstream signaling pathways (Bhattacharya et al., 2011). Signaling pathways are not static linear pathways that simply transmit signals, but are responsible for encoding and integrating both internal and external cues (Kholodenko, 2006). Exposure to chemical insults can perturb the dynamic and highly coordinated signaling pathways responsible for normal biological function and maintenance (Houck and Kavlock, 2008). Additionally, chemical insult at one molecular target and pathway can propagate throughout the signaling network due to pathway crosstalk and the interconnectivity of various signaling cascades (Kholodenko et al., 2010). As such, cellular spatiotemporal signaling dynamics are responsible for integrating and interpreting intra- and extracellular cues to make cellular fate decisions, such as proliferation, differentiation, or programmed cell death (apoptosis) (Murphy et al., 2004; von Kriegsheim et al., 2009; Kholodenko et al., 2010).

Signaling networks are primarily regulated by post-translational modifications (PTMs). PTMs are vital to signaling coordination and diversification of proteins for various functions (Wold, 1981; Aye-Han et al., 2009). PTMs can modify proteins after translation via complex molecules (glycosylation and isoprenylation), peptides or proteins (ubiquitylation and sumoylation), chemical groups (acetylation, methylation, and phosphorylation), and cleavage (proteolysis) (Wang et al., 2014). The rapid toxicodynamic response of cells to xenobiotics is primarily coordinated by signal transduction networks, which follow a simple framework: the phosphorylation / dephosphorylation cycle mediated by kinases and phosphatases (Kumar et al., 2007; Schilling et al., 2009). Kinases are intimately involved in the regulation of signaling events relevant to cellular death and survival processes (Bononi et al., 2011). Xenobiotics 
can interfere with kinase signaling via activation (e.g., overstimulation) or inhibition (Boelsterli, 2007). Kinases can be activated/deactivated via the addition or removal of a phosphate group at serine, threonine, and tyrosine residues (-OH containing amino acids) (Wold, 1981), shown in Figure 1.3.

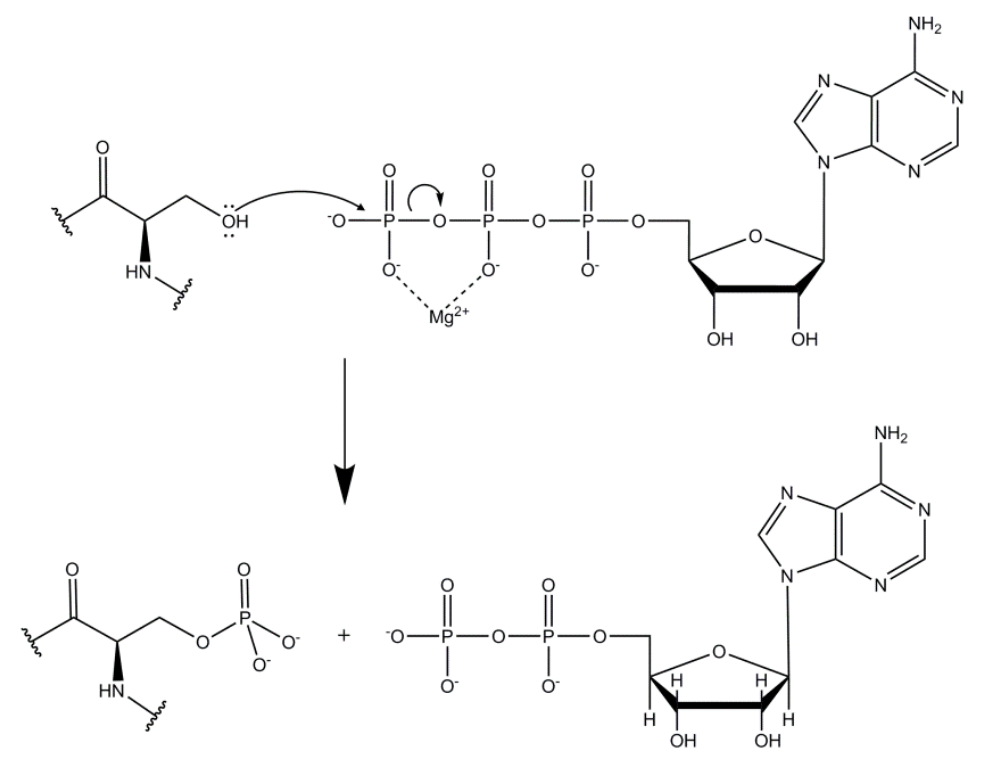

Figure 1.3. Mechanism of phosphorylation. This reaction scheme uses serine as the example residue, however, this mechanism is true for tyrosine and threonine phosphorylation as well.

The phosphorylation/dephosphorylation of a protein acts as a molecular switch to activate or deactivate a protein. In the catalytic domain of protein kinases, the $-\mathrm{OH}$ group on serine, threonine, and tyrosine residues act as a nucleophile, attacking the $\gamma$-phosphate on adenosine triphosphate (ATP) resulting in the transfer of a phosphoryl group to the protein (Endicott et al., 2012). In this scheme, magnesium is a critical component for protein phosphorylation, acting as a supportive chelator. The covalent bonding between phosphate groups and protein kinases typically induce a conformational change, aiding or preventing protein-protein (enzyme-substrate) interactions (Wang et al., 2012). It is this 
protein-protein/enzyme-substrate interaction where critical cellular information can be transmitted throughout the signaling network.

Under xenobiotic stress conditions, many pathways can be perturbed, including survival and death pathways. The three major stress-activated pathways are the mitogenactivated protein kinase (MAPK) pathway (also commonly referred to as extracellular signal-regulated kinases (ERK) pathway), the stress-activated protein kinase (SAPK) pathway (also commonly referred to as jun N-terminal kinase (JNK) pathway), and the p38 pathway (Paul et al., 1997; Tibbles and Woodgett, 1999; Pouyssegur et al., 2002). These stress pathways are endogenously active, controlling cellular fate via transcriptional activation and/or inhibition of genes regulating survival/proliferation and cell death (Boelsterli, 2007). These pathways were traditionally thought of as discrete linear signaling cascades; however, it is now well known that they are merely components of a dynamic and highly interconnected network of pathways that contain many crosstalk, feedback and feedforward mechanisms to adequately and efficiently response to stress-inducing stimuli (Junttila et al., 2008; Kholodenko et al., 2010). Therefore, it is the delicate balance/imbalance of these pathways that decides the cell's ultimate fate (Bononi et al., 2011; Currie et al., 2014). The highly dynamic and interconnected nature of signaling networks has made it increasingly difficult to elucidate and predict network responses to xenobiotic stress. However, advances in computational and network biology, high-throughput experimental techniques, such as -omics investigations using mass spectrometry, multiplex bead-based ELISA (enzyme-linked immunosorbent assay) suspension array systems, and microchip arrays, and public databases have greatly improved signaling research. With these advances, new 
toxicological risk assessment approaches for determining potential toxic outcome of a xenobiotic or mixture using mechanistic information can be attempted, as postulated by the NRC report (NRC, 2007).

\subsubsection{Bioenergetic changes post-exposure}

Kinase signaling is an energy demanding process, and its reliance on phosphorylation results in the consumption of substantial amounts of available adenosine triphosphate (ATP) (Hammerman et al., 2004). ATP-production governs ATP-consuming processes, such as signal transduction in mammalian cells, and this production is primarily driven by oxidative phosphorylation within mitochondria (Buttgereit and Brand, 1995; Ainscow and Brand, 1999). Mitochondria are the energy production hub of a cell via production of ATP via phosphorylation and, of equal importance, key mediators in kinase signal transduction, regulating cell survival, proliferation, differentiation, and death (Hammerman et al., 2004; Mohamed et al., 2014). Due to their critical role in many cellular processes, mitochondria are also susceptible to xenobiotic exposure effects. Additionally, irreversible processes leading to cell death primarily rely on two mitochondria-related phenomena: 1) the inability to reverse mitochondrial dysfunction, resulting in ATP depletion and 2) the disturbance of membrane function (both mitochondrial membrane and plasma membrane) (Law and Elmore, 2008). Therefore, maintenance of mitochondrial bioenergetics and integrity is critical to cellular fate.

Mitochondria are comprised of several key features: the outer mitochondrial membrane, intermembrane space, inner mitochondrial membrane, and matrix (Figure 


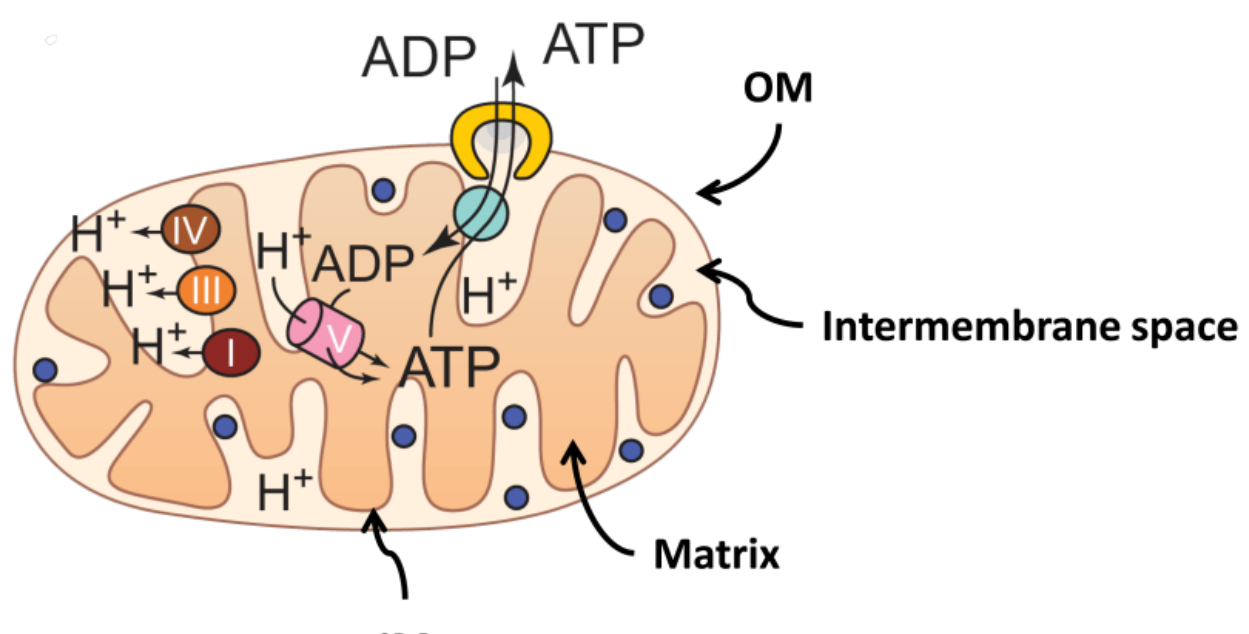

IM

Figure 1.4. Mitochondria. The two membranes of mitochondria are shown above as the outer membrane (OM) and inner membrane (IM). The VDAC transmembrane protein is shown in yellow at the OM and ANT transmembrane protein is in teal at the IM to illustrate the shuttle of ATP from ATP synthase (also known as Complex V) of the ETC to the cytosol. Small blue dots indicate cytochrome c within the intermembrane space and roman numeral cartoons indicate ETC subunits. Adapted from Desagher and Martinou (2000), reprinted with permission from Elsevier.

1.4). The outer mitochondrial membrane is permeable to small molecules (molecular weight $<5,000 \mathrm{~g} / \mathrm{mol}$ ) and ions, which readily diffuse through transmembrane porin proteins, such as voltage-gated anion channel (VDAC) (Ninomiya-Tsuji, 2008). The inner membrane, however, is impermeable to most molecules and ions, including protons and ATP (Lehninger et al., 2008). The inner membrane contains the proteins that make up the electron transport chain (ETC). Additionally, the inner membrane contains numerous transport proteins to allow metabolites to pass through into the matrix and to export ATP generated by the electron transport chain (ETC) into the intermembrane space (Ninomiya-Tsuji, 2008). The space between the two membranes is called the intermembrane space. The intermembrane contains a small heme-containing protein called cytochrome $\mathrm{c}$ within the intermembrane-side of inner membrane folds (referred to as intracristae space), which acts as an electron carrier for Complex III on the ETC, and when released from the outer membrane, can initiate caspase-dependent apoptosis 
(Desagher and Martinou, 2000; Kroemer et al., 2007). The protein adenine nucleotide translocase (ANT) is the transporter responsible for shuttling ATP from the matrix to the intermembrane space (Ninomiya-Tsuji, 2008). Additionally, ANT forms a complex with VDAC (an outer mitochondrial membrane transporter), commonly referred to as the permeability transition pore (PTP) that can compromise the impermeability of the mitochondrial membranes. If the PTP is open, an influx of ions and water can bombard the matrix, causing swelling, loss of membrane potential, and uncoupling of oxidative phosphorylation, eventually leading to cell death (Fosslien, 2001). Finally, the matrix, which is in the space contained by the inner membrane, houses all energy-yielding oxidative reactions, such as the citric acid cycle, fatty acid oxidation, and the ETC (Ninomiya-Tsuji, 2008). The matrix also contains important ions (magnesium, calcium, and potassium), metabolic intermediates, ATP/ADP, and mitochondrial DNA. Mitochondrial DNA genes are transcribed and translated within the matrix as well.

The number of mitochondria in each eukaryotic cell can vary depending upon the cell type. Red blood cells (erythrocytes) do not have mitochondria, however, the heart, kidney, and liver are considered mitochondria-rich (Veltri et al., 1990). Mitochondriarich cell types are especially sensitive to xenobiotics that target the ETC. There are two classes of ETC inhibitors: 1) xenobiotics that block the transport of electrons via binding to ETC enzyme complexes (e.g., deguelin binding to Complex I) and 2) xenobiotics that stimulate the flow of electrons at one portion of the ETC, but shunt electrons away from their normal route by acting as an electron acceptor. Disruption of normal ETC function and mitochondrial bioenergetics can have deleterious effects (both acute and chronic) on the target tissue as well as whole organism. Examples of pathological conditions where 
mitochondrial dysfunction plays a critical role are neurodegenerative diseases, neurotoxicity, heart disease (myocardial infarction and atherosclerosis), liver injury (ischemic injury and cholestasis), obesity, and cancer (Kroemer et al., 2007; Nunnari and Suomalainen, 2012). Thus, mitochondrial bioenergetics should be carefully considered and included in toxicity risk assessment.

\subsubsection{Timescale of exposure effects}

The toxicity of a xenobiotic or mixture at their intended molecular/tissue target(s) can vary over exposure time and any change is commonly known as time-dependent toxicity (Dawson et al., 2014). The initial toxicodynamic response to any xenobiotic or mixture exposure is predominantly coordinated by signal transduction networks, which can initiate response within the first few seconds to minutes of exposure. The time course from initial toxicodynamic response(s) to cell death following exposure can have a vast range (Rehm et al., 2009). For example, the time delay between xenobiotic exposure and execution of apoptosis can take from several hours to over a day (Lemasters et al., 1998; Messam and Pittman, 1998). With this in mind, monitoring early cellular changes that contribute to adaptive stress response (survival and new homeostatic state) or lead to adverse effects (apoptosis, carcinogenicity) may aid toxicological understanding and ultimately the prediction of potential adverse effects from xenobiotic or mixture exposures. 


\subsection{Experimentally testing early cellular changes that may contribute to exposure sensing and response}

Traditional risk assessment testing techniques involve screening potential agents using in vivo and in vitro endpoint experiments, such as neurotoxicity or developmental toxicity, and mode of action analysis, such as cytotoxicity or mutagenicity (Dix et al., 2007). However, the recent paradigm shift charged by the NRC report has initiated a new chemical risk assessment approach that utilizes high-throughput in vitro screening assays to exploit early cellular changes (such as signaling pathway perturbations and alterations in cellular bioenergetics) to reveal mechanistic information about adverse or adaptive effects after xenobiotic exposure.

\subsubsection{In vitro cell culture}

The large number of potential toxic chemical agents that have yet to be fully characterized pose a significant problem for risk assessment testing (NRC, 2007). Traditional in vivo methods cannot be solely relied on for risk assessment testing due to the low-throughput, high financial and time cost, and the sheer number of animals necessary to test the thousands of chemicals yet to be evaluated. Further, the utility of mechanistic information from animal studies has been questionable due to disappointing cross-species extrapolation for real life low dose human exposure effects (Houck and Kavlock, 2008). Thus, a paradigm shift from traditional in vivo methods to an in vitro approach utilizing assays to collect mechanistic information for pathway/network analyses and eventual prediction modeling with computational toxicology has been 
initiated by the NRC report (NRC, 2007). The NRC suggested human in vitro highthroughput screening assays to measure early cellular effects, such as perturbations of critical signaling pathways related to survival and death (referred to as potential adverse outcome pathways by the NRC), across a wide dosing range and multiple cell lines, both immortalized and primary cell culture (Andersen et al., 2010).

The paradigm shift from traditional in vivo animal studies to new in vitro highthroughput screening assays using human cell lines raises some new questions. What cell type should be used (immortalized vs primary)? Primary cell culture is culture that is initiated immediately following tissue extraction from the sample organism. Once the cells reach confluency (typically $80 \%$ of the culture-containing flask covered by cells, without overlapping each other), cells need to be subcultured (also known as passaging) by dividing the cells into multiple culture flasks for continued growth. Primary cell culture can typically only be subcultured several times before they can no longer be used. On the other hand, immortalized (continuous) culture is when cells are cultured for a theoretically infinite number of subcultures, which is achieved via transformation (spontaneously transformed via cancerous cell lines or chemically induced immortalization). Immortalized/continuous cell lines offer a low cost, reproducible in vitro experimental set-up, but may result in disparate responses than those achieved in primary culture or in vivo due to their continuous cell cycle progression. Even though the response may be slightly different from immortalized to primary or in vivo studies, useful mechanistic information can be collected for toxicity risk assessment. Fortunately, there are several cell lines commercially available that have libraries of data, such as HepG2 (human hepatocellular carcinoma) for liver toxicity (O'Brien et al., 2006), MCF-7 
(human breast cancer) for estrogen responsive toxicity studies (Holliday and Speirs, 2011), and HEK293 (human embryonic kidney cells) for kidney toxicity (Sasaki et al., 2007).

Liver injury and toxicity due to xenobiotic exposure is a major concern for pharmaceutical regulatory and toxicological risk assessment (Kavlock et al., 2012). Additionally, the liver plays a vital role in xenobiotic biotransformation after exposure (Mersch-Sundermann et al., 2004). Thus, in vitro high-throughput approaches using human-derived liver cell lines to probe important early cellular exposure effects and potential pathway perturbations are necessary for toxicological risk assessment. The U.S. EPA ToxCast research program was initiated to screen previously untested environmental chemicals for adverse effects using a large number of high-throughput bioassays. Data collected from ToxCast assays is made available through the ToxCast data library to disseminate chemical exposure profiles to further toxicology research and gain a mechanistic understanding of chemical exposure (Sipes et al., 2013). The ToxCast library has two liver models for toxicity testing: primary rat hepatocytes and HepG2 cells (Kavlock et al., 2012). Both models offer important information about hepatotoxicity. Due to the limited availability of human primary liver cells, rat primary hepatocytes are often used for hepatotoxicity risk assessments. Previous studies have shown that xenobiotic metabolizing enzymes have significant interspecies variation (Selkirk, 1977; Maslansky and Williams, 1982). An accepted alternative to primary rat hepatocytes for liver toxicity research is the HepG2 cell line. A benefit of using HepG2 vs primary rat hepatocytes is that they are human-derived. Most importantly, the HepG2 cell line retains endogenous xenobiotic metabolizing enzymes, whereas primary hepatocyte culture 
typically loses these vital enzymes (Knasmuller et al., 1998). The in vitro studies discussed in Chapters 2 - 4 use the HepG2 cell line as the model system for several xenobiotic exposure proof-of-principle approaches.

\subsubsection{Real time in vitro assays to measure early cellular changes}

As discussed previously, initial cellular responses to xenobiotic exposure are rapid, dynamic, and highly integrated for determining eventual cellular fate. Assays capable of capturing these dynamic processes and relating them to apical outcomes (cell death, survival, plasma membrane degradation) are necessary for xenobiotic risk assessment predictions. The real-time assays used in this dissertation are related to mitochondrial bioenergetics (reduced form of nicotinamide adenine dinucleotide (NADH) production, cellular oxygen consumption, and estimations of ATP from NADH and oxygen consumption) and cell death (plasma membrane degradation).

\subsubsection{Mitochondrial bioenergetics assays: NADH generation and oxygen consumption}

$\mathrm{NADH}$ is a vital component of mitochondrial function, energy metabolism and oxidative stress (Ying, 2008). As a critical component of the ETC and bioenergetics, early cellular effects and perturbations can be monitored via changes in cellular NADH. One of the most useful and remarkable features of NADH is that it strongly absorbs at $340 \mathrm{~nm}$; therefore, real time kinetic measurements of cellular NADH can be obtained 
without the assistance of any fluorescent tag or probe (McComb et al., 1976; Vrana et al., 2014).

Intracellular molecular oxygen is a key component of cellular homeostasis and mitochondrial oxidative phosphorylation via the ETC. After xenobiotic exposure, early cellular changes associated with mitochondrial bioenergetics and cellular respiration can be monitored using real-time oxygen consumption assays. Determining key time points related to perturbations in cellular respiration can further the mechanistic understanding of xenobiotic exposure and eventual cell survival/death. High-throughput real-time assays for oxygen consumption that do not perturb endogenous intracellular activity are limited. The most popular method for cellular oxygen consumption is the Clark electrode, however it is a very low-throughput method (one sample at a time) (Diepart et al., 2010). The development of oxygen sensitive extracellular probes that can reproducibly measure discrete changes in oxygen consumption over time (with comparable sensitivity to the Clark electrode) have significantly advanced mitochondrial and toxicological research. Once such probe was developed by Luxcel Corp (Cork, Ireland), manufactured as MitoXpress. The MitoXpress probe is an extracellular phosphorescent platinumcoproporphyrin dye with a long emission time and stable phosphorescent signal that can be used for 24 hour kinetic measurements in a 96-well plate format, making it highthroughput (Dmitriev et al., 2010). For this assay, the MitoXpress probe is quenched by molecular oxygen $\left(\mathrm{O}_{2}\right)$. A decrease in extracellular $\mathrm{O}_{2}$ concentration (increase in cellular oxygen consumption) is measured as an increase in signal (less probe is quenched by $\mathrm{O}_{2}$ ), whereas an increase in extracellular $\mathrm{O}_{2}$ concentration is measured as a decrease in signal (more $\mathrm{O}_{2}$ to quench the probe; less oxygen is consumed by the cell) (Diepart et al., 2010). 
Finally, one of the most critical components of bioenergetics, ATP can be measured to monitor cellular perturbations after xenobiotic exposure. Previously, assays for intracellular ATP have employed fluorescent tags, which can potentially disrupt endogenous intracellular activity (e.g., FRET) (Berg et al., 2009), or involve cell lysis (e.g., luciferase assay), making real-time in vitro ATP measurements not possible (Imamura et al., 2009). Monitoring relative ATP generation in response to chemical insult without potentially disrupting sensitive intracellular activity is imperative. To this end, the Boyd Lab has developed an extracellular approach to estimate ATP production with data collected from real-time oxygen consumption and NADH production assays. These data sets allow for stoichiometric determinations of ATP production in real-time. Theoretically, mitochondrial oxidative phosphorylation is responsible for producing a substantial portion of cellular ATP, and traditionally, NADH and oxygen are related to ATP production as shown below (Kadenbach, 1986; Lehninger et al., 2008):

$$
1 \mathrm{NADH}+\frac{1}{2} \mathrm{O}_{2}=3 \mathrm{ATP}
$$

Since our MitoXpress assay monitors oxygen consumption, the equation can be doubled:

$$
2 \mathrm{NADH}+\mathrm{O}_{2}=6 \mathrm{ATP}
$$

Additionally, cells can produce ATP via glycolysis, where 1 ATP is generated for each available NADH. This may be particularly true for HepG2 cells, since many cancer cells have been shown to have a high reliance on glycolysis due to the Warburg effect (Warburg, 1956; Vander Heiden et al., 2009). To account for ATP production when there 
is limited or no oxygen, an "if then else loop" was used to calculate theoretical ATP generation:

$$
\begin{aligned}
& \text { If } 6 \times\left[\mathrm{O}_{2}\right]_{\text {sample }}-2 \times[\mathrm{NADH}]_{\text {sample }}>0 ; \\
& \text { Then } \frac{\left[6 \times\left[\mathrm{O}_{2}\right]_{\text {sample }}\right]-\left[2 \times[\mathrm{NADH}]_{\text {sample }}\right]}{4} \\
& \text { Else } \frac{[\mathrm{NADH}]_{\text {sample }}}{4}
\end{aligned}
$$

This method has proven successful for two disparate xenobiotics, demonstrating a strong correlation to relative ATP measurements collected via the luciferase assay (Vrana et al., 2014).

\subsubsection{Plasma membrane degradation}

The "point-of-no-return" for which a cell decides to die has yet to be determined (Kroemer et al., 2005). However, certain cellular features implicate a cell has died; one such feature is the loss of plasma membrane integrity (Kroemer et al., 2005). A simple, reproducible, and high-throughput assay for plasma membrane degradation is the ethidium homodimer-1 (EthD-1) assay, commonly referred to as a "dead assay." The EthD-1 dye is impermeable for cells with an intact plasma membrane. However, when the plasma membrane is permeabilized, the EthD- 1 can penetrate the cell and intercalate with DNA nucleic acids. When EthD-1 is bound to strands/segments of DNA, the dye emits a strong red fluorescence, whereas in the absence of available DNA segments, the dye has a very low intensity fluorescent signal (Grogan et al., 2002). This dye is not only useful for in vitro plate reader-based assays, but also fluorescence microscopy. 


\subsubsection{Endpoint in vitro assays to measure early cellular changes and long term exposure}

effects

The previous assays described are best suited for in vitro, real-time kinetic measurements using a high-throughput platform, such as 96+ well plate assays. However, assays that require cell lysis or disruption of cellular function are commonly referred to as endpoint assays. The high-throughput in vitro endpoint assays used in this work are MTT (cell viability), luciferin/luciferase (intracellular ATP), and multiplex bead-based ELISA (phosphoprotein or cytokine).

\subsubsection{Viability}

There are several viability measurements used for in vitro experiments that are cheap, easy to use, and high-throughput. Examples include the lactate dehydrogenase (LDH) assay, neutral red, and methyl tetrazolium (MTT) assay. There are benefits and drawbacks for each method, however, of the three, neutral red and MTT are the most sensitive for cell viability (Fotakis and Timbrell, 2006). While all three assays are used to determine viability, most are used as an orthogonal measurement of cytotoxicity (i.e., not used alone), or for initial screening of appropriate dosing ranges (Galluzzi et al., 2009). The only true assays for cell death measure plasma membrane degradation, such as the EthD-1 dye. However, due to its low cost, robustness, and ease of use, MTT is commonly included in studies to estimate viability. The MTT reagent, 3-[4,5-dimethylthiazol-2-yl]2,5-diphenyltetrazolium bromide, is a yellow water soluble salt that is converted into insoluble formazan crystals in the presence of succinate dehydrogenase within the 
mitochondria of metabolically active cells via cleavage of the tetrazolium ring (Fotakis and Timbrell, 2006). The insoluble purple formazan crystals can be solubilized by DMSO or detergent for quantification via absorbance using 96-well plate spectrophotometry.

\subsubsection{Intracellular ATP}

As described previously, ATP is a critical biomolecule for cell survival. While real-time assays for ATP generation are limited, endpoint assays for ATP are widely used and extremely sensitive (Imamura et al., 2009). Additionally, ADP:ATP ratios have been used to measure cell viability, apoptosis, and necrosis (Bradbury et al., 2000). The predominant endpoint ATP assay is the D-luciferin-luciferase assay, which requires cell lysis for in vitro applications. The luciferase assay is a bioluminescent assay where the substrate D-luciferin is converted into oxyluciferin, by the luciferase enzyme in the presence of ATP. The conversion from luciferin to oxyluciferin emits a stable, relatively long-lived light emission ( $\sim 30$ minutes). This assay is very sensitive, ranging from $10^{-13}$ mol to $>10^{-6}$ mol ATP, and can be used for a wide variety of sample matrices, such as soil, milk, plasma, and cell culture (Kricka, 1988).

\subsubsection{Multiplex bead-based suspension array}

The analysis of key signal transduction perturbations via PTM assays is one of the most valuable tools for intracellular exposure mechanism-based risk assessment. Upon insult by a xenobiotic, the cellular toxicodynamic response is coordinated by signal 
transduction networks, which follow a rudimentary framework, the phosphorylation / dephosphorylation cycle mediated by kinases and phosphatases (Kumar et al., 2007; Schilling et al., 2009). Traditionally, western blot and immunoprecipitation have been the assays of choice for phosphoprotein analyses (Schmelzle and White, 2006). While western blotting has been the gold standard for phosphoprotein determinations, it is very low throughput. A multiplexed and high-throughput assay for phosphoprotein analysis to elucidate network perturbations and mechanistic exposure information is the bead-based (microsphere) sandwich-based ELISA, developed by Luminex (Poetz et al., 2010). This assay is a modification of a traditional ELISA, whereby the capture antibody $(\mathrm{Ab})$ is immobilized to magnetic (or non-magnetic) polystyrene microspheres (Figure 1.5).

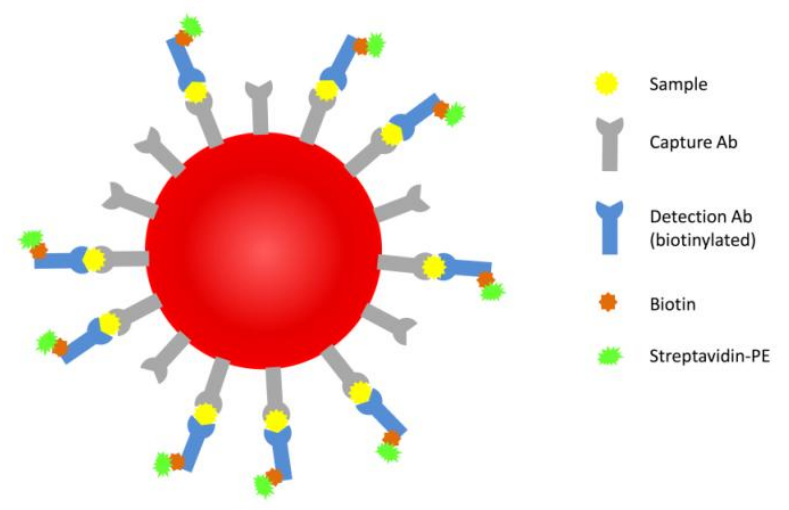

Figure 1.5. Bead-based ELISA. A microsphere, which can be magnetic or non-magnetic, (shown in red) has two fluorescent dyes (excited by the same wavelength of light but emit different wavelengths) encapsulated within the bead. These dyes act as the bead recognition element, and the ratio of dyes identifies the bead type (e.g., bead region 1 has a ratio of 1:100 for the two dyes, and this region has the Ab for ERK protein immobilized on the surface). The assay proceeds as a traditional sandwich ELISA (with the exception that the capture $\mathrm{Ab}$ is immobilized to a microsphere) where the capture $\mathrm{Ab}$ is specific for one of your desired analytes (e.g, anti-ERK Ab is selective for ERK protein) and the biotinylated detection Ab recognizes a separate epitope of your desired analyte (e.g., anti-phosphoERK $\mathrm{Ab}$ to recognize phosphorylated ERK). The biotinylated detection $\mathrm{Ab}$ then binds with the fluorescent reporter, streptavidin-phycoerythrin (due to the extremely high binding affinity of biotin for streptavidin). Antibody, Ab; phycoerythrin (PE). 
The assay workflow proceeds as follows: 1) capture Ab beads are added to the assay plate wells 2) add the analyte(s)-containing sample and incubate 3) add biotinylated detection $\mathrm{Ab}$ and incubate 4) add fluorescent reporter (e.g., streptavidin-phycoerythrin complex) and incubate 5) analyze samples via suspension array (Houser, 2012).

Since this is a multiplex method, 100+ different Ab-bead combinations can be assayed per well. The beads are coded with two dyes that can be excited by the same wavelength but emit different wavelengths. Depending upon the ratio of the two encapsulated dyes, the bead region (code) can be measured by the instrument. In order to differentiate between bead dyes and fluorescent reporter dye, the suspension array platform utilizes a duel laser system: a red solid state laser $(635 \mathrm{~nm})$ to excite dyes encapsulated within the microspheres for bead type identification and a Nd:YAG laser $(532 \mathrm{~nm})$ to excite the phycoerythrin dye (fluorescent reporter). The fluorescent reporter is bound to the analyte-bead complex (shown in green, Figure 1.5) for quantitation of the captured analyte (Houser, 2012). All emission intensities are detected with a photomultiplier tube. This method is high-throughput and requires low sample volume. Currently, Luminex-manufactured magnetic microspheres (sold under the name MagPlex) have 500 unique bead regions, allowing for 500 different antibody assays to be performed per well of a 96-well plate (Luminex, 2015).

\subsection{References}

Ainscow, E.K., Brand, M.D., 1999. Top-down control analysis of ATP turnover, glycolysis and oxidative phosphorylation in rat hepatocytes. Eur J Biochem 263, 671-685. 
Allen, B.C., Kavlock, R.J., Kimmel, C.A., Faustman, E.M., 1994. Dose-response assessment for developmental toxicity. II. Comparison of generic benchmark dose estimates with no observed adverse effect levels. Fundam Appl Toxicol 23, 487495.

Andersen, M.E., 2010. Calling on science: making "alternatives" the new gold standard. ALTEX 27, 29-37.

Andersen, M.E., Al-Zoughool, M., Croteau, M., Westphal, M., Krewski, D., 2010. The future of toxicity testing. J Toxicol Environ Health B Crit Rev 13, 163-196.

Andersen, M.E., Dennison, J.E., Thomas, R.S., Conolly, R.B., 2005. New directions in incidence-dose modeling. Trends Biotechnol 23, 122-127.

Andersen, M.E., Krewski, D., 2009. Toxicity testing in the 21st century: bringing the vision to life. Toxicol Sci 107, 324-330.

Aptula, A.O., Roberts, D.W., 2006. Mechanistic applicability domains for nonanimalbased prediction of toxicological end points: general principles and application to reactive toxicity. Chem Res Toxicol 19, 1097-1105.

ATSDR, 2001a. Guidance for the Preparation of an Interaction Profile. U.S. Department of Health and Human Services, Public Health Service Agency for Toxic Substance and Disease Registry, Atlanta, GA, USA, pp.

ATSDR, 2001b. Guidance Manual for the Assessment of Joint Toxic Action of Chemical Mixtures. Final/Technical Report. U.S. Department of Health and Human Services, Publich Health Service Agency for Toxic Substance and Disease Registry, Atlanta, GA, USA, pp.

Attene-Ramos, M.S., Miller, N., Huang, R., Michael, S., Itkin, M., Kavlock, R.J., Austin, C.P., Shinn, P., Simeonov, A., Tice, R.R., Xia, M., 2013. The Tox21 robotic platform for the assessment of environmental chemicals--from vision to reality. Drug Discov Today 18, 716-723.

Aye-Han, N.N., Ni, Q., Zhang, J., 2009. Fluorescent biosensors for real-time tracking of post-translational modification dynamics. Curr Opin Chem Biol 13, 392-397.

Berenbaum, M.C., 1989. What is synergy? Pharmacol Rev 41, 93-141. 
Berg, J., Hung, Y.P., Yellen, G., 2009. A genetically encoded fluorescent reporter of ATP:ADP ratio. Nat Methods 6, 161-166.

Bhattacharya, S., Zhang, Q., Carmichael, P.L., Boekelheide, K., Andersen, M.E., 2011. Toxicity testing in the 21 century: defining new risk assessment approaches based on perturbation of intracellular toxicity pathways. PLoS One 6, e20887.

Bliss, C.I., 1939. The toxicity of poisons applied jointly. Ann Appl Biol 26, 585-615.

Boelsterli, U.A., 2007. Mechanistic Toxicology: The Molecular Basis of How Chemicals Disrupt Biological Targets, Second Edition. CRC Press, Boca Raton, FL.

Bononi, A., Agnoletto, C., De Marchi, E., Marchi, S., Patergnani, S., Bonora, M., Giorgi, C., Missiroli, S., Poletti, F., Rimessi, A., Pinton, P., 2011. Protein kinases and phosphatases in the control of cell fate. Enzyme Res 2011, 329098.

Borgert, C.J., Quill, T.F., McCarty, L.S., Mason, A.M., 2004. Can mode of action predict mixture toxicity for risk assessment? Toxicol Appl Pharmacol 201, 85-96.

Boyd, J., Saksena, A., Patrone, J.B., Williams, H.N., Boggs, N., Le, H., Theodore, M., 2011. Exploring the boundaries of additivity: mixtures of NADH: quinone oxidoreductase inhibitors. Chem Res Toxicol 24, 1242-1250.

Bradbury, D.A., Simmons, T.D., Slater, K.J., Crouch, S.P., 2000. Measurement of the ADP:ATP ratio in human leukaemic cell lines can be used as an indicator of cell viability, necrosis and apoptosis. J Immunol Methods 240, 79-92.

Butterworth, B.E., Conolly, R.B., Morgan, K.T., 1995. A strategy for establishing mode of action of chemical carcinogens as a guide for approaches to risk assessments. Cancer Lett 93, 129-146.

Buttgereit, F., Brand, M.D., 1995. A hierarchy of ATP-consuming processes in mammalian cells. Biochem J 312 ( Pt 1), 163-167.

Calabrese, E.J., 2001. Overcompensation stimulation: a mechanism for hormetic effects. Crit Rev Toxicol 31, 425-470. 
Calabrese, E.J., 2008. Hormesis: why it is important to toxicology and toxicologists. Environ Toxicol Chem 27, 1451-1474.

Calabrese, E.J., 2009. Getting the dose-response wrong: why hormesis became marginalized and the threshold model accepted. Arch Toxicol 83, 227-247.

Calabrese, E.J., Baldwin, L.A., 2003a. The hormetic dose-response model is more common than the threshold model in toxicology. Toxicol Sci 71, 246-250.

Calabrese, E.J., Baldwin, L.A., 2003b. Toxicology rethinks its central belief. Nature 421, 691-692.

Casarett, L.J., Klaassen, C.D., 2008. Casarett and Doull's toxicology : the basic science of poisons. McGraw-Hill Medical, New York.

Cedergreen, N., Christensen, A.M., Kamper, A., Kudsk, P., Mathiassen, S.K., Streibig, J.C., Sorensen, H., 2008. A review of independent action compared to concentration addition as reference models for mixtures of compounds with different molecular target sites. Environ Toxicol Chem 27, 1621-1632.

Cox, C., 1987. Threshold dose-response models in toxicology. Biometrics 43, 511-523.

Currie, H.N., Vrana, J.A., Han, A.A., Scardoni, G., Boggs, N., Boyd, J.W., 2014. An approach to investigate intracellular protein network responses. Chem Res Toxicol 27, 17-26.

Dawson, D.A., Allen, E.M., Allen, J.L., Baumann, H.J., Bensinger, H.M., Genco, N., Guinn, D., Hull, M.W., Il'Giovine, Z.J., Kaminski, C.M., Peyton, J.R., Schultz, T.W., Poch, G., 2014. Time-dependence in mixture toxicity prediction. Toxicology 326, 153-163.

Dellarco, V.L., Wiltse, J.A., 1998. US Environmental Protection Agency's revised guidelines for Carcinogen Risk Assessment: incorporating mode of action data. Mutat Res 405, 273-277.

Desagher, S., Martinou, J.C., 2000. Mitochondria as the central control point of apoptosis. Trends Cell Biol 10, 369-377. 
Diepart, C., Verrax, J., Calderon, P.B., Feron, O., Jordan, B.F., Gallez, B., 2010. Comparison of methods for measuring oxygen consumption in tumor cells in vitro. Anal Biochem 396, 250-256.

Dix, D.J., Houck, K.A., Martin, M.T., Richard, A.M., Setzer, R.W., Kavlock, R.J., 2007. The ToxCast program for prioritizing toxicity testing of environmental chemicals. Toxicol Sci 95, 5-12.

Dmitriev, R.I., Zhdanov, A.V., Ponomarev, G.V., Yashunski, D.V., Papkovsky, D.B., 2010. Intracellular oxygen-sensitive phosphorescent probes based on cellpenetrating peptides. Anal Biochem 398, 24-33.

Edwards, I.R., Aronson, J.K., 2000. Adverse drug reactions: definitions, diagnosis, and management. Lancet 356, 1255-1259.

Endicott, J.A., Noble, M.E., Johnson, L.N., 2012. The structural basis for control of eukaryotic protein kinases. Annu Rev Biochem 81, 587-613.

Feron, V.J., Groten, J.P., van Bladeren, P.J., 1998. Exposure of humans to complex chemical mixtures: hazard identification and risk assessment. Arch Toxicol Suppl 20, 363-373.

Forget, J., Pavillon, J.-F., Beliaeff, B., Bocquene, G., 1999. Joint action of pollutant combinations (pesticides and metals) on survival (LC50 values) and acetylcholinesterase activity of Tigriopus brevicornis (Copepoda, Harpacticoida). Environ Toxicol Chem 18, 912-918.

Fosslien, E., 2001. Mitochondrial medicine--molecular pathology of defective oxidative phosphorylation. Ann Clin Lab Sci 31, 25-67.

Fotakis, G., Timbrell, J.A., 2006. In vitro cytotoxicity assays: comparison of LDH, neutral red, MTT and protein assay in hepatoma cell lines following exposure to cadmium chloride. Toxicol Lett 160, 171-177.

Galluzzi, L., Aaronson, S.A., Abrams, J., Alnemri, E.S., Andrews, D.W., Baehrecke, E.H., Bazan, N.G., Blagosklonny, M.V., Blomgren, K., Borner, C., Bredesen, D.E., Brenner, C., Castedo, M., Cidlowski, J.A., Ciechanover, A., Cohen, G.M., De Laurenzi, V., De Maria, R., Deshmukh, M., Dynlacht, B.D., El-Deiry, W.S., Flavell, R.A., Fulda, S., Garrido, C., Golstein, P., Gougeon, M.L., Green, D.R., Gronemeyer, H., Hajnoczky, G., Hardwick, J.M., Hengartner, M.O., Ichijo, H., 
Jaattela, M., Kepp, O., Kimchi, A., Klionsky, D.J., Knight, R.A., Kornbluth, S., Kumar, S., Levine, B., Lipton, S.A., Lugli, E., Madeo, F., Malomi, W., Marine, J.C., Martin, S.J., Medema, J.P., Mehlen, P., Melino, G., Moll, U.M., Morselli, E., Nagata, S., Nicholson, D.W., Nicotera, P., Nunez, G., Oren, M., Penninger, J., Pervaiz, S., Peter, M.E., Piacentini, M., Prehn, J.H., Puthalakath, H., Rabinovich, G.A., Rizzuto, R., Rodrigues, C.M., Rubinsztein, D.C., Rudel, T., Scorrano, L., Simon, H.U., Steller, H., Tschopp, J., Tsujimoto, Y., Vandenabeele, P., Vitale, I., Vousden, K.H., Youle, R.J., Yuan, J., Zhivotovsky, B., Kroemer, G., 2009. Guidelines for the use and interpretation of assays for monitoring cell death in higher eukaryotes. Cell Death Differ 16, 1093-1107.

Gennings, C., Carter, W., Campain, J., Bae, D., Yang, R., 2002. Statistical analysis of interactive cytotoxicity in human epidermal ke. J Agric Biol Environ Stat 7, 5873.

Grogan, S.P., Aklin, B., Frenz, M., Brunner, T., Schaffner, T., Mainil-Varlet, P., 2002. In vitro model for the study of necrosis and apoptosis in native cartilage. J Pathol 198, 5-13.

Groten, J.P., Feron, V.J., Suhnel, J., 2001. Toxicology of simple and complex mixtures. Trends Pharmacol Sci 22, 316-322.

Hammerman, P.S., Fox, C.J., Thompson, C.B., 2004. Beginnings of a signal-transduction pathway for bioenergetic control of cell survival. Trends Biochem Sci 29, 586592.

Holliday, D.L., Speirs, V., 2011. Choosing the right cell line for breast cancer research. Breast Cancer Res 13, 215.

Houck, K.A., Kavlock, R.J., 2008. Understanding mechanisms of toxicity: insights from drug discovery research. Toxicol Appl Pharmacol 227, 163-178.

Houser, B., 2012. Bio-Rad's Bio-Plex ${ }^{\circledR}$ suspension array system, xMAP technology overview. Arch Physiol Biochem 118, 192-196.

Imamura, H., Nhat, K.P., Togawa, H., Saito, K., Iino, R., Kato-Yamada, Y., Nagai, T., Noji, H., 2009. Visualization of ATP levels inside single living cells with fluorescence resonance energy transfer-based genetically encoded indicators. Proc Natl Acad Sci U S A 106, 15651-15656. 
Jonker, M.J., Piskiewicz, A.M., Ivorra i Castella, N., Kammenga, J.E., 2004. Toxicity of binary mixtures of cadmium-copper and carbendazim-copper to the nematode Caenorhabditis elegans. Environ Toxicol Chem 23, 1529-1537.

Jonker, M.J., Svendsen, C., Bedaux, J.J., Bongers, M., Kammenga, J.E., 2005. Significance testing of synergistic/antagonistic, dose level-dependent, or dose ratio-dependent effects in mixture dose-response analysis. Environ Toxicol Chem 24, 2701-2713.

Junttila, M.R., Li, S.P., Westermarck, J., 2008. Phosphatase-mediated crosstalk between MAPK signaling pathways in the regulation of cell survival. FASEB J 22, 954965.

Kadenbach, B., 1986. Regulation of respiration and ATP synthesis in higher organisms: hypothesis. J Bioenerg Biomembr 18, 39-54.

Kavlock, R., Chandler, K., Houck, K., Hunter, S., Judson, R., Kleinstreuer, N., Knudsen, T., Martin, M., Padilla, S., Reif, D., Richard, A., Rotroff, D., Sipes, N., Dix, D., 2012. Update on EPA's ToxCast program: providing high throughput decision support tools for chemical risk management. Chem Res Toxicol 25, 1287-1302.

Kholodenko, B.N., 2006. Cell-signalling dynamics in time and space. Nat Rev Mol Cell Biol 7, 165-176.

Kholodenko, B.N., Hancock, J.F., Kolch, W., 2010. Signalling ballet in space and time. Nat Rev Mol Cell Biol 11, 414-426.

Knasmuller, S., Parzefall, W., Sanyal, R., Ecker, S., Schwab, C., Uhl, M., MerschSundermann, V., Williamson, G., Hietsch, G., Langer, T., Darroudi, F., Natarajan, A.T., 1998. Use of metabolically competent human hepatoma cells for the detection of mutagens and antimutagens. Mutat Res 402, 185-202.

Kricka, L.J., 1988. Clinical and biochemical applications of luciferases and luciferins. Anal Biochem 175, 14-21.

Kroemer, G., El-Deiry, W.S., Golstein, P., Peter, M.E., Vaux, D., Vandenabeele, P., Zhivotovsky, B., Blagosklonny, M.V., Malorni, W., Knight, R.A., Piacentini, M., Nagata, S., Melino, G., 2005. Classification of cell death: recommendations of the Nomenclature Committee on Cell Death. Cell Death Differ 12 Suppl 2, 14631467. 
Kroemer, G., Galluzzi, L., Brenner, C., 2007. Mitochondrial membrane permeabilization in cell death. Physiol Rev 87, 99-163.

Kumar, D., Srikanth, R., Ahlfors, H., Lahesmaa, R., Rao, K.V., 2007. Capturing cell-fate decisions from the molecular signatures of a. Mol Syst Biol 3, 150.

Law, M., Elmore, S., 2008. Mechanisms of Cell Death. In Smart, R.C., Hodgson, E., (Eds.), Molecular and Biochemical Toxicology. John Wiley \& Sons, Hoboken, N.J., pp.

Lehninger, A., Cox, M.M., Nelson, D.L., 2008. Lehninger Principles of Biochemistry. W. H. Freeman, New York.

Lemasters, J.J., Nieminen, A.L., Qian, T., Trost, L.C., Elmore, S.P., Nishimura, Y., Crowe, R.A., Cascio, W.E., Bradham, C.A., Brenner, D.A., Herman, B., 1998. The mitochondrial permeability transition in cell death: a common mechanism in necrosis, apoptosis and autophagy. Biochim Biophys Acta 1366, 177-196.

Loewe, S., Muischnek, H., 1926. Effect of combinations: mathematical basis of problem. Arch Exp Pathol Pharmakol 114, 313-326.

Luminex, 2015. MagPlex Microspheres - Luminex Corporation. http://www.luminexcorp.com/Products/ReagentsMicrospheres/MagPlex_Microsp heres/, pp.

Maslansky, C.J., Williams, G.M., 1982. Primary cultures and the levels of cytochrome P450 in hepatocytes from mouse, rat, hamster, and rabbit liver. In Vitro 18, 683693.

McCarty, L.S., Borgert, C.J., 2006. Review of the toxicity of chemical mixtures: Theory, policy, and regulatory practice. Regul Toxicol Pharmacol 45, 119-143.

McComb, R.B., Bond, L.W., Burnett, R.W., Keech, R.C., Bowers, G.N., Jr., 1976. Determination of the molar absorptivity of NADH. Clin Chem 22, 141-150.

Meled, M., Thrasyvoulou, A., Belzunces, L.P., 1998. Seasonal variations in susceptibility of Apis mellifera to the synergistic action of prochloraz and deltamethrin. Environ Toxicol Chem 17, 2517-2520. 
Mersch-Sundermann, V., Knasmuller, S., Wu, X.J., Darroudi, F., Kassie, F., 2004. Use of a human-derived liver cell line for the detection of cytoprotective, antigenotoxic and cogenotoxic agents. Toxicology 198, 329-340.

Messam, C.A., Pittman, R.N., 1998. Asynchrony and commitment to die during apoptosis. Exp Cell Res 238, 389-398.

Mohamed, S.M., Veeranarayanan, S., Minegishi, H., Sakamoto, Y., Shimane, Y., Nagaoka, Y., Aki, A., Poulose, A.C., Echigo, A., Yoshida, Y., Maekawa, T., Kumar, D.S., 2014. Cytological and Subcellular Response of Cells Exposed to the Type-1 RIP Curcin and its Hemocompatibility Analysis. Scientific Reports 4.

Murphy, L.O., MacKeigan, J.P., Blenis, J., 2004. A network of immediate early gene products propagates subtle differences in mitogen-activated protein kinase signal amplitude and duration. Mol Cell Biol 24, 144-153.

Ninomiya-Tsuji, J., 2008. Mitochondrial Dysfunction. In Smart, R.C., Hodgson, E., (Eds.), Molecular and Biochemical Toxicology. John Wiley \& Sons, Hoboken, N.J., pp.

NRC, 2007. Toxicity Testing in the 21st Century: A Vision and a Strategy. National Academies Press, Washington, D.C.

Nunnari, J., Suomalainen, A., 2012. Mitochondria: in sickness and in health. Cell 148, 1145-1159.

O'Brien, P.J., Irwin, W., Diaz, D., Howard-Cofield, E., Krejsa, C.M., Slaughter, M.R., Gao, B., Kaludercic, N., Angeline, A., Bernardi, P., Brain, P., Hougham, C., 2006. High concordance of drug-induced human hepatotoxicity with in vitro cytotoxicity measured in a novel cell-based model using high content screening. Arch Toxicol 80, 580-604.

Paul, A., Wilson, S., Belham, C.M., Robinson, C.J., Scott, P.H., Gould, G.W., Plevin, R., 1997. Stress-activated protein kinases: activation, regulation and function. Cell Signal 9, 403-410.

Poetz, O., Henzler, T., Hartmann, M., Kazmaier, C., Templin, M.F., Herget, T., Joos, T.O., 2010. Sequential Multiplex Analyte Capturing for Phosphoprotein Profiling*. Mol Cell Proteomics 9, 2474-2481. 
Posthuma, L., Baerselman, R., Van Veen, R.P., Dirven-Van Breemen, E.M., 1997. Single and joint toxic effects of copper and zinc on reproduction of Enchytraeus crypticus in relation to sorption of metals in soils. Ecotoxicol Environ Saf 38, 108-121.

Pottenger, L.H., Gollapudi, B.B., 2009. A case for a new paradigm in genetic toxicology testing. Mutat Res 678, 148-151.

Pouyssegur, J., Volmat, V., Lenormand, P., 2002. Fidelity and spatio-temporal control in MAP kinase (ERKs) signalling. Biochem Pharmacol 64, 755-763.

Rajapakse, N., Ong, D., Kortenkamp, A., 2001. Defining the impact of weakly estrogenic chemicals on the action of steroidal estrogens. Toxicol Sci 60, 296-304.

Rehm, M., Huber, H.J., Hellwig, C.T., Anguissola, S., Dussmann, H., Prehn, J.H., 2009. Dynamics of outer mitochondrial membrane permeabilization during apoptosis. Cell Death Differ 16, 613-623.

Sasaki, A., Oshima, Y., Fujimura, A., 2007. An approach to elucidate potential mechanism of renal toxicity of arsenic trioxide. Exp Hematol 35, 252-262.

Schilling, M., Maiwald, T., Hengl, S., Winter, D., Kreutz, C., Kolch, W., Lehmann, W.D., Timmer, J., Klingmuller, U., 2009. Theoretical and experimental analysis links isoform-specific ERK signalling to. Mol Syst Biol 5, 334.

Schlosser, P.M., Bogdanffy, M.S., 1999. Determining modes of action for biologically based risk assessments. Regul Toxicol Pharmacol 30, 75-79.

Schmelzle, K., White, F.M., 2006. Phosphoproteomic approaches to elucidate cellular signaling networks. Curr Opin Biotechnol 17, 406-414.

Selkirk, J.K., 1977. Divergence of metabolic activation systems for short-term mutagenesis assays. Nature 270, 604-607.

Sipes, N.S., Martin, M.T., Kothiya, P., Reif, D.M., Judson, R.S., Richard, A.M., Houck, K.A., Dix, D.J., Kavlock, R.J., Knudsen, T.B., 2013. Profiling 976 ToxCast chemicals across 331 enzymatic and receptor signaling assays. Chem Res Toxicol 26, 878-895. 
Spurgeon, D.J., Jones, O.A., Dorne, J.L., Svendsen, C., Swain, S., Sturzenbaum, S.R., 2010. Systems toxicology approaches for understanding the joint effects of environmental chemical mixtures. Sci Total Environ 408, 3725-3734.

Tibbles, L.A., Woodgett, J.R., 1999. The stress-activated protein kinase pathways. Cell Mol Life Sci 55, 1230-1254.

USEPA, 1986. Guidelines for the Health Risk Assessment of Chemical Mixtures U.S. Evironmental Protection Agency. In Assessment, U.E.N.C.f.E., (Ed.), Washington, D.C., pp.

USEPA, 1999. Guidance for Identifying Pesticide Chemicals That Have a Common Mechanism of Toxicity U.S. Environmental Protection Agency, Washington, D.C., pp.

USEPA, 2000. Supplementary Guidance for Conducting Health Risk Assessment of Chemical Mixtures, Washington, D.C., pp.

USEPA, 2002. Guidance on Cumulative Risk Assessment of Pesticide Chemicals That Have a Common Mechansim of Toxicity, Washington, D.C., pp.

Van Gestel, C.A.M., Hensbergen, P.J., 1997. Interaction of $\mathrm{Cd}$ and $\mathrm{Zn}$ toxicity for Folsomia candida Willem (Collembola:Isotomidae) in relation to bioavailability in soil. Environ Toxicol Chem 16, 1177-1186.

Veltri, K.L., Espiritu, M., Singh, G., 1990. Distinct genomic copy number in mitochondria of different mammalian organs. J Cell Physiol 143, 160-164.

von Kriegsheim, A., Baiocchi, D., Birtwistle, M., Sumpton, D., Bienvenut, W., Morrice, N., Yamada, K., Lamond, A., Kalna, G., Orton, R., Gilbert, D., Kolch, W., 2009. Cell fate decisions are specified by the dynamic ERK interactome. Nat Cell Biol 11, 1458-1464.

Vrana, J.A., Currie, H.N., Han, A.A., Boyd, J., 2014. Forecasting cell death doseresponse from early signal transduction responses in vitro. Toxicol Sci 140, 338351.

Wang, Y., Eddy, J.A., Price, N.D., 2012. Reconstruction of genome-scale metabolic models for 126 human tissues using mCADRE. BMC Syst Biol 6, 153. 
Wang, Y.C., Peterson, S.E., Loring, J.F., 2014. Protein post-translational modifications and regulation of pluripotency in human stem cells, Cell Res, England, pp. 143160.

Wold, F., 1981. In vivo chemical modification of proteins (post-translational modification). Annu Rev Biochem 50, 783-814.

Ying, W., 2008. NAD+/NADH and NADP+/NADPH in cellular functions and cell death: regulation and biological consequences. Antioxid Redox Signal 10, 179-206. 
Chapter 2

Forecasting cell death dose-response to single chemicals

in vitro 


\section{Forecasting cell death dose-response to single chemicals in vitro ${ }^{1}$}

The rapid pharmacodynamic response of cells to toxic xenobiotics is primarily coordinated by signal transduction networks, which follow a simple framework: the phosphorylation / dephosphorylation cycle mediated by kinases and phosphatases. However, the time course from initial pharmacodynamic response(s) to cell death following exposure can have a vast range. Viewing this time lag between early signaling events and the ultimate cellular response as an opportunity, we hypothesize that monitoring the phosphorylation of proteins related to cell death and survival pathways at key, early time-points may be used to forecast a cell's eventual fate, provided that we can measure and accurately interpret the protein responses. In this paper, we focused on a three-phased approach to forecast cell death after exposure: 1) determine time-points relevant to important signaling events (protein phosphorylation) by using estimations of ATP production to reflect the relationship between mitochondrial-driven energy metabolism and kinase response, 2) experimentally determine phosphorylation values for proteins related to cell death and/or survival pathways at these significant time-points, and 3) use cluster analysis to predict the dose-response relationship between cellular exposure to a xenobiotic and plasma membrane degradation at 24 hours post-exposure. To test this approach, we exposed HepG2 cells to two disparate treatments: a GSK-3 $\beta$ inhibitor and a MEK inhibitor. After using our three-phased approach, we were able to accurately forecast the 24 hour HepG2 plasma membrane degradation dose-response

\footnotetext{
${ }^{1}$ Parts of this chapter have been published previously, either in part or in full, from Vrana, J.A., Currie, H.N., Han, A.A., Boyd, J. (2014) Forecasting cell death dose-response from early signal transduction responses in vitro. Toxicol Sci. 140, 338-351. Reproduced with permission from Oxford University Press.
} 
from protein phosphorylation values as early as 20 minutes post-MEK inhibitor exposure and 40 minutes post-GSK-3 $\beta$ exposure.

\subsection{Introduction}

In mammalian systems, the cellular response to toxic xenobiotics involves the complex, yet coordinated transmission of intracellular signaling molecules to determine a cell's eventual fate (i.e., survival or death) (Kumar et al., 2007; Schilling et al., 2009). The initial pharmacodynamic response to any xenobiotic exposure is predominantly coordinated by signal transduction networks, which can initiate response within the first few seconds to minutes of exposure. This response follows a simple framework: the phosphorylation and dephosphorylation cycle of proteins mediated by kinases and phosphatases (Kholodenko, 2006; Newman et al., 2013). However, the time course of cell death from exposure to visual morphological changes can have a vast range. For example, the time delay between xenobiotic exposure and execution of apoptosis can take from several hours to over a day (Lemasters et al., 1998; Messam and Pittman, 1998). Based upon the time delay between these early and late responses, we hypothesize that monitoring the phosphorylation of proteins related to cell death and survival pathways may be predictive of a cell's eventual fate, provided that we can read and correctly interpret the protein responses.

Considering the vast number of proteins involved in critical intracellular signaling processes, the number and type of proteins to be monitored for forecasting a cell's fate must be judiciously selected. An advantageous group of proteins to monitor for this 
approach are kinases involved in the regulation of signaling events relevant to cellular death and survival processes. Irrespective of the cell death modality, the cellular decision to survive or die relies on the integration of signals at mitochondrial membranes that contribute to mitochondrial membrane permeability (MMP) (Kroemer et al., 2007). By monitoring kinases upstream of MMP signaling proteins, a detailed snapshot of the cell's own interpretation of exposure can theoretically be determined, and this early signaling may foreshadow the cell's fate long before the final steps of cell death occur.

Appropriately capturing the time course of critical signaling events that will be predictive of cellular survival or death post-exposure is difficult. However, time-points of significant deviation may be determined by exploiting the intricate inter-relationship between mitochondrial-driven energy metabolism and kinase response in real-time. Most importantly, this can be accomplished without potentially disrupting intracellular activity. Kinase signaling is an energy demanding process, and its reliance on phosphorylation results in the consumption of substantial amounts of available adenosine triphosphate (ATP) (Hammerman et al., 2004). ATP-production governs ATP-consuming processes, such as signal transduction in mammalian cells, and this production is primarily driven by oxidative phosphorylation within mitochondria (Buttgereit and Brand, 1995; Ainscow and Brand, 1999). Additionally, it is known that some types of cell death (e.g., apoptosis and autophagy) require appreciable amounts of energy due to their reliance on ATPdependent processes (Yamamoto et al., 1998; Eguchi et al., 1999; Kanzawa et al., 2004), such as activation of caspase signaling cascades (Leist et al., 1997), chromatin condensation (Eguchi et al., 1997), and autophagic sequestration (Kondo et al., 2005). Therefore, by monitoring energy production processes in the cell in real-time, key time 
points of significant deviation may indicate critical kinase signaling events relevant to, in this study, cell death post-exposure.

In this paper, we focused on a three-phased approach to forecast cell death in an in vitro system: 1) determine time-points relevant to critical signaling events, 2) experimentally determine the phosphorylation of proteins related to cell death and/or survival at these significant time-points, and 3) use cluster analysis to predict the 24 hour plasma membrane degradation dose-response of cells to xenobiotic exposure. We chose the human hepatocellular carcinoma-derived HepG2 cell line as our model in vitro system because the liver is rich in mitochondria (Veltri et al., 1990) and this cell line is human-derived. Additionally, hepatocellular carcinoma incidence is on the rise in the United States with 5-year survival rates of $10 \%$ (Altekruse et al., 2009). To test this approach, we chose xenobiotics that have activities at disparate intracellular targets in vitro. Specifically, we exposed HepG2 cells to various doses of 4-Benzyl-2-methyl-1,2,4-

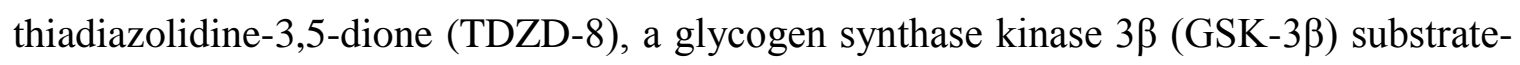
competitive inhibitor (Martinez et al., 2002). As a therapeutic target, GSK3 has seen a recent surge in interest for its multifunctional role in a variety of signaling processes, as well as its implication in a number of diseases, such as diabetes, Alzheimer's, bipolar disorder, and cancer (Kim and Kimmel, 2000; Wang et al., 2006). We also exposed HepG2 cells to various doses of 2-Chloro-3-(N-succinimidyl)-1,4-naphthoquinone (MEK inh II), a MEK1 inhibitor (Bakare et al., 2003). MEK1 has been shown to be constitutively active in several carcinoma cell lines, such as hepatocellular carcinoma, contributing to cell survival (Ballif and Blenis, 2001; Mitsui et al., 2001). This makes the MEK1-MAPK signal transduction pathway an attractive target for chemotherapies 
(Huynh et al., 2003). By using our three-phased approach, we were able to accurately forecast the 24 hour HepG2 plasma membrane degradation dose-response from protein phosphorylation values as early as 20 minutes post-MEK inh II exposure and 40 minutes post-TDZD-8 exposure. The implications of this proof of principle study may have a broad reaching impact: from the ability to forecast in vitro cytotoxicity of xenobiotic exposures to the identification of therapeutic windows for pharmacological treatments.

\subsection{Materials and Methods}

\subsubsection{Materials}

4-Benzyl-2-methyl-1,2,4-thiadiazolidine-3,5-dione (TDZD-8, cas 327036-89-5), Dulbecco's modified Eagle's medium (DMEM), sodium pyruvate, D-glucose, Lglutamate, and sodium bicarbonate were obtained from Sigma Aldrich (St. Louis, MO). 2-Chloro-3-(N-succinimidyl)-1,4-naphthoquinone (MEK inh II, cas 623163-52-0) was purchased from CalBiochem (La Jolla, CA, USA). HEPES was purchased from Fisher Scientific (USA). Fetal bovine serum, Ethidium homodimer-1 cytotoxicity kit, ATP determination kit (luciferase assay), and penicillin-streptomycin were obtained from Invitrogen (Carlsbad, CA). HyClone phosphate buffered saline (PBS) was purchased from Thermo Scientific (USA). Cell lines and MTT assay kits were obtained from American Type Culture Collection (Manassas, VA). MitoXpress oxygen probe was obtained from Luxcel Corporation (Cork, Ireland). Deionized water used in this study was prepared with the Milli-Q Water System (Millipore, Bedford, MA). 


\subsubsection{Cell culture}

Human hepatocellular carcinoma-derived HepG2 cells were cultured in DMEM, supplemented with $2 \mathrm{~g} / \mathrm{L}$ D-glucose, $2 \mathrm{mM}$ L-Glutamate, $5 \mathrm{mM}$ HEPES, $24 \mathrm{mM}$ sodium bicarbonate, $1 \mathrm{mM}$ sodium pyruvate, $10 \%$ fetal bovine serum, $100 \mathrm{U} / \mathrm{mL}$ penicillin, and $100 \mathrm{mg} / \mathrm{mL}$ streptomycin. Cells were maintained in a humidified atmosphere at $37^{\circ} \mathrm{C}, 5 \%$ $\mathrm{CO}_{2}$ and passaged at $80 \%$ confluence.

\subsubsection{Dosimetry}

For MTT, NADH, cellular ATP, and plasma membrane degradation assays, cells were seeded into clear-bottom, 96-well plates (black-sided for fluorescence assays) at a concentration of $4 \times 10^{4}$ cells per well in DMEM without phenol red and allowed to grow for 24 hours before dosing. For multiplex phosphoprotein assays, cells were seeded in 12well plates at a concentration of $5 \times 10^{5}$ cells per well in DMEM without phenol red and allowed to grow for 24 hours before dosing. For oxygen consumption assays, cells were seeded into clear-bottom, black-sided 96-well plates at a concentration of $8 \times 10^{4}$ cells per well in DMEM without phenol red and allowed to grow for 24 hours before dosing. Medium was then aspirated from wells and cells were challenged with TDZD-8 or MEK inh II. TDZD-8 and MEK inh II were prepared so that resulting well concentrations would be $<1 \%$ DMSO. 


\subsubsection{MTT assay}

After 24 hours of exposure to TDZD-8 $(10,20,30,40,50$, or $100 \mu \mathrm{M})$ or MEK inh II $(1,5,10,20,50$, or $100 \mu \mathrm{M})$, cell viability was determined using the MTT (3-(4,5dimethyl)-2,5-diphenyl tetrazolium bromide) assay, according to the manufacturer's protocol. This assay is based on the reduction of tetrazolium MTT to formazan by metabolically active cells, in part by the action of dehydrogenase enzymes, to generate reducing equivalents such as NADH and NADPH. Briefly, MTT reagent was added to the wells of the microplate, and after four hours of incubation at $37^{\circ} \mathrm{C}$, intracellular formazan crystals were solubilized with the provided detergent solution. Absorbance values were obtained using the Tecan InfiniteM1000 plate reader (Tecan US, Raleigh, NC) with a measurement wavelength of $570 \mathrm{~nm}$ and a reference wavelength of $700 \mathrm{~nm}$, read from the bottom. Assay was performed in quadruplicate. Relative viability was calculated by subtracting the negative control (contained no cells) and normalizing to controls, which received dosing vehicle (<1\% DMSO).

\subsubsection{Oxygen consumption assay}

Immediately after dosing with TDZD-8 $(10,20,30,40$, or $50 \mu \mathrm{M})$ or MEK inh II $(1,5,10,20$, or $50 \mu \mathrm{M})$, cellular oxygen consumption was assessed using the MitoXpress probe, according to manufacturer's protocol. Briefly, oxygen-sensitive probe was diluted to a stock concentration of $1 \mu \mathrm{M}$, and stock probe was diluted 1:15 in each well of a black walled 96 -well plate containing cells $\left(8 \times 10^{4}\right.$ cells/well); $100 \mu \mathrm{L}$ of pre-warmed mineral oil was also added to each well to block ambient oxygen from the cells. After pre- 
warming the plates at $37^{\circ} \mathrm{C}$ for 1 hour, cells were challenged with varying doses of TDZD-8 or MEK inh II. Immediately following addition of TDZD-8 or MEK inh II, oxygen consumption was determined by measuring fluorescence. Fluorescent signal was obtained using the Infinite M1000 microplate reader (Tecan US, Raleigh NC) with excitation wavelength of $380 \mathrm{~nm}$ and emission wavelength of $650 \mathrm{~nm}$, reading from the bottom every 10 minutes for 24 hours after dosing. Experiments were performed in quadruplicate. Relative oxygen consumption was calculated by normalizing to controls, which received dosing vehicle $(<1 \%$ DMSO).

\subsubsection{NADH assay}

Immediately after dosing with TDZD-8 $(10,20,30,40$, or $50 \mu \mathrm{M})$ or MEK inh II $(1,5,10,20$, or $50 \mu \mathrm{M})$, NADH generation was determined by measuring absorbance. The absorbance of NADH at wavelength $340 \mathrm{~nm}$ was measured every 10 minutes for 24 hours. Experiments were performed in quadruplicate. Relative NADH generation was calculated by normalizing to controls, which received dosing vehicle (<1\% DMSO).

\subsubsection{Cellular ATP assay}

The boiling water method was used to extract cellular ATP from attached cells, as described previously (Yang et al., 2002). After 40 minutes, 10 hours, or 24 hours of exposure to TDZD-8 $(10,20,30,40$, or $50 \mu \mathrm{M})$, medium was aspirated and cells were washed twice with PBS. In the same manner, after 20 minutes, 8 hours 20 minutes, or 24 hours of exposure to MEK inh II $(1,5,10,20$, or $50 \mu \mathrm{M})$, medium was aspirated and 
cells were washed twice with PBS. Cells were suspended in $100 \mu \mathrm{L} /$ well boiling deionized water with repeated pipetting. The cell suspension was transferred to a microcentrifuge tube and centrifuged $12,000 \mathrm{~g}$ for 5 minutes at $4^{\circ} \mathrm{C}$. Following centrifugation, $10 \mu \mathrm{L}$ of supernatant was used for the ATP luciferase assay, which was performed according to the manufacturer's protocol. Bioluminescence was measured using the Infinite M1000 microplate reader (Tecan US, Raleigh NC). Experiments were performed in quadruplicate. Relative cellular ATP was determined by normalizing to positive control, which received dosing vehicle (<1\% DMSO).

\subsubsection{Multiplex phosphoprotein assay}

After 40 minutes or 10 hours of exposure to TDZD-8 (10, 20, 30, 40, 50, or 100 $\mu \mathrm{M})$, cells were washed with ice cold PBS and lysed according to manufacturer's protocol. In the same manner, after 20 minutes or 8 hours 20 minutes of exposure to MEK inh II $(1,5,10,20,50$, or $100 \mu \mathrm{M})$, cells were washed with ice cold PBS and lysed according to manufacturer's protocol. Total protein concentration was determined using the DC Protein Assay (BioRad, Hercules, CA) according to the manufacturer's instructions. Protein phosphorylation was determined by using the bead-based BioPlex suspension array system (Bio-Rad, Hercules, CA) and lysates were prepared according to the manufacturer's protocol. Beads and detection antibodies against phosphorylated AKT (Ser473), ERK1/2(Thr202/Tyr204, Thr185/Tyr187), GSK-3 $\alpha / \beta$ (Ser21/Ser9), HSP27 (Ser78), IкB $\alpha$ (Ser32/Ser36), JNK1/2 (Thr 183/Tyr185), MEK1 (Ser217/Ser221), p38MAPK (Thr180/Tyr182), p53 (Ser15), and p90RSK (Thr359/Ser363) were obtained 
from Bio-Rad (Hercules, CA). Beads and detection antibodies against phosphorylated BAD (Ser 112), CREB1 (Ser133), IGFR (Tyr1131), IRS1 (Ser636/Ser639), EGFR (Tyr), MSK1(Ser212), p70S6K (Thr421/Ser424), AKT(Thr308), and p53(Ser46) were obtained from EMD Millipore. Relative phosphorylation was calculated by normalizing to control, which received dosing vehicle $(<1 \%$ DMSO). TDZD-8 exposed phosphoprotein experiments were performed in triplicate and MEK inh II exposed phosphoprotein experiments were performed in duplicate.

\subsubsection{Plasma membrane degradation assay}

To determine plasma membrane degradation of HepG2 cells exposed to TDZD-8 or MEK inh II, the ethidium homodimer-1 (EthD-1) kinetic assay was used. Twenty-four hours after seeding HepG2 cells $\left(4 \times 10^{4}\right)$ in a black-sided clear-bottom 96 well plate, culture medium was removed and replaced with probe-containing culture medium ( $2 \mu \mathrm{M}$ EthD-1). Dead control wells were exposed to $70 \%$ methanol and the plate was incubated for 30 minutes. Following the 30 minute incubation, cells were challenged with TDZD-8 $(10,20,30,40,50$, or $100 \mu \mathrm{M})$ or MEK inh II $(1,5,10,20,50$, or $100 \mu \mathrm{M})$ and read immediately after dosing. Fluorescent signal was obtained using the Infinite M1000 microplate reader (Tecan US, Raleigh NC) with excitation wavelength of $530 \mathrm{~nm}$ and emission wavelength of $645 \mathrm{~nm}$, reading from the bottom every 10 minutes for 24 hours after dosing. Experiments were performed in sextuplicate. Relative plasma membrane degradation was determined by normalizing to dead control (received $70 \%$ methanol). 


\subsubsection{Statistical Analysis}

Dose-response curves for MTT assays were generated by best-fit Hill-plot regression with varying slope of scatter plot data using Prism V5 (Graphpad Software, San Diego, CA). Selection of time points related to critical signaling events (40 minutes and 10 hours for TDZD-8; 20 minutes and 8 hours 20 minutes for MEK inh II) were determined by estimating temporal bifurcation points from theoretical ATP generation and activity using SAS JMP V12Pro (Cary, NC). Correlation analysis for theoretical ATP generation and relative cellular ATP was determined using SAS JMP V12Pro (Cary, NC). Two-way hierarchical cluster analyses with Ward's minimum variance were determined by using SAS JMP V12Pro (Cary, NC). Statistical significance for plasma membrane degradation (observed and forecasted) was determined by using a two-way analysis of variance (ANOVA) with Bonferroni post-test. A difference of $P<0.05$ was considered statistically significant. For viability, plasma membrane degradation (observed and predicted), cellular ATP, and relative phosphorylation data, error bars reflect standard error of the mean (S.E.M.).

\subsubsection{Signaling Pathway Analysis}

Functional pathway and network analyses were generated with Ingenuity Pathways Analysis (IPA) software (Ingenuity Systems) as described elsewhere (MadozGurpide et al., 2007). IPA identified pathways from the IPA Library of Canonical Pathways that were significant to our phosphorylation data. The 19 proteins used in this paper were considered for analysis. For this study, significance between our protein 
phosphorylation data set (TDZD-8 and MEK inh II) and the molecular and cellular function p-score, along with associated network functions, was measured by Fisher's exact test. The Fisher's exact test calculates a p-value to determine the probability that the relationship between the phosphorylated proteins in our data set and a canonical pathway, biological function or disease could be related by chance alone. We reported the top three biological functions associated with our datasets.

\subsection{Results}

\subsubsection{HepG2 viability in response to TDZD-8 and MEK inh II}

For this proof-of-principle study, we first determined the appropriate dosing range for TDZD-8, a GSK-3 $\beta$ substrate-competitive inhibitor $\left(\mathrm{IC}_{50}=2 \mu \mathrm{M}\right)$ (Martinez et al., 2002), and MEK inh II, a MEK1 inhibitor $\left(\mathrm{IC}_{50}=0.38 \mu \mathrm{M}\right)$ (Bakare et al., 2003). Previously, MEK inh II has also been shown to inhibit other intracellular targets, such as ERK1 $\left(\mathrm{IC}_{50}=82.9 \mu \mathrm{M}\right)$ and $\operatorname{Raf1}\left(\mathrm{IC}_{50}=34.5 \mu \mathrm{M}\right)($ Bakare et al., 2003). To accomplish this, we measured HepG2 viability via MTT assay in response to varying doses of TDZD-8 and MEK inh II at 24 hours post-exposure (Figures 2.1a,b). From HepG2 viability, the best-fit Hill-plot regression with varying slope was used to determine the $\mathrm{EC}_{50}$ of TDZD-8 $(19 \pm 1 \mu \mathrm{M})$ and MEK inh II $(9 \pm 1 \mu \mathrm{M})$. 


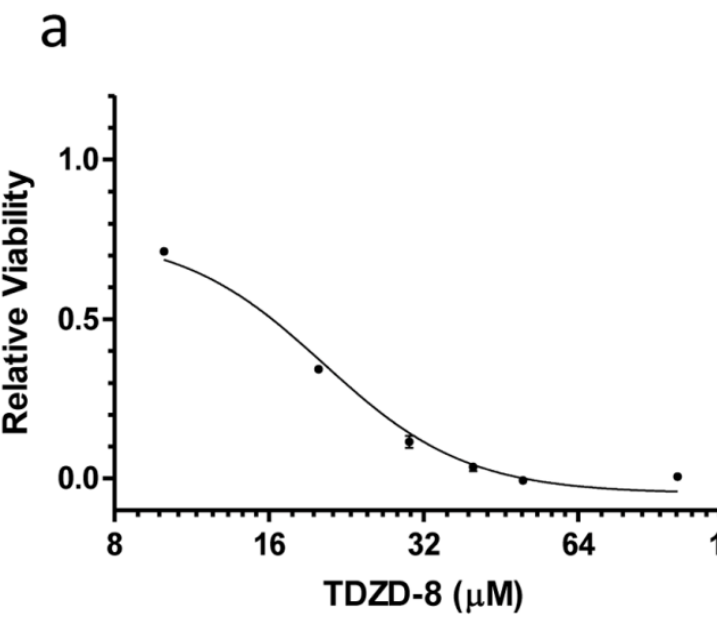

b

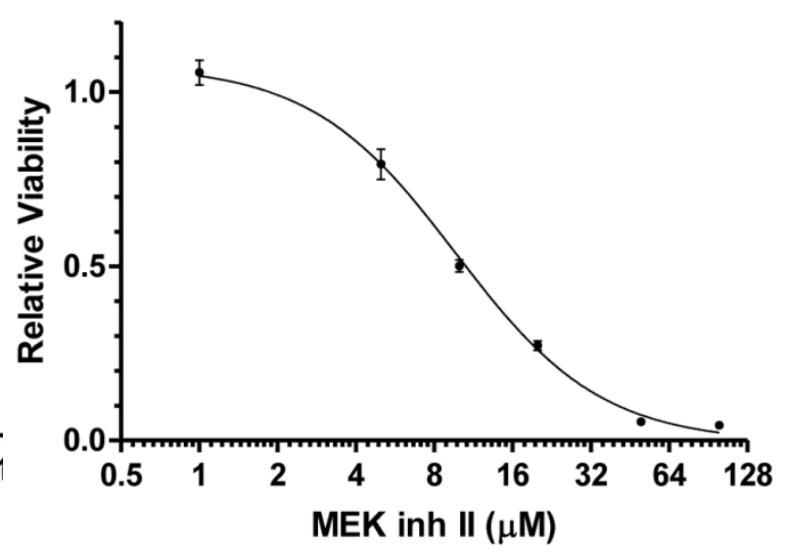

Figure 2.1. HepG2 viability to determine relevant TDZD-8 and MEK inh II doses. HepG2 viability dose-response curves for a) TDZD-8 and b) MEK inh II. Viability was measured by MTT assay and is shown as relative viability. Relative viability was calculated relative to control cells, which received dosing vehicle ( $<1 \%$ DMSO), but no inhibitor. Dose-response curves were generated using the best-fit Hill-plot regression with varying slope. The $\mathrm{x}$-axis is shown as $\log 2$ to better visualize TDZD-8 and MEK inh II dosing range. Error bars reflect \pm S.E.M.

\subsubsection{Estimation of ATP generation to determine critical signaling events}

Monitoring relative ATP generation in response to chemical insult without potentially disrupting sensitive intracellular activity is imperative. To this end, we have developed an extracellular approach to determine kinase signaling events on a temporal scale by estimating ATP production with data collected from real-time oxygen consumption and NADH production assays. These data sets allow for stoichiometric determinations of ATP production in real-time. Theoretically, mitochondrial oxidative phosphorylation is responsible for producing a substantial portion of cellular ATP, and traditionally, NADH and oxygen are related to ATP production as shown below (Kadenbach, 1986; Nelson and Cox, 2008):

$$
1 \mathrm{NADH}+\frac{1}{2} \mathrm{O}_{2}=3 A T P
$$

Since our assay monitors oxygen consumption, the equation can be doubled: 


$$
2 \mathrm{NADH}+\mathrm{O}_{2}=6 \mathrm{ATP}
$$

Additionally, cells can produce ATP via glycolysis, where 1 ATP is generated for each available NADH. This may be particularly true for HepG2 cells, since many cancer cells have been shown to have a high reliance on glycolysis due to the Warburg effect (Warburg, 1956; Vander Heiden et al., 2009). To account for ATP production when there is limited or no oxygen, an "if then else loop" was used to calculate theoretical ATP generation:

$$
\begin{aligned}
& \text { If } 6 \times\left\{\mathrm{O}_{2}\right\}_{\text {sample }}-2 \times\{\mathrm{NADH}\}_{\text {sample }}>0 ; \\
& \text { Then } \frac{\left[6 \times\left\{\mathrm{O}_{2}\right\}_{\text {sample }}\right]-\left[2 \times\{\mathrm{NADH}\}_{\text {sample }}\right]}{4} \\
& \text { Else } \frac{\{\mathrm{NADH}\}_{\text {sample }}}{4}
\end{aligned}
$$

To account for variations in ATP generation over time, we calculated the relative ATP generation activity (equation 3), which we believe more accurately captures temporal bifurcations relevant to critical signaling events. This equation is the average of the integrated $\mathrm{NADH}$ and $\mathrm{O}_{2}$ consumption assays over time divided by their total NADH produced and $\mathrm{O}_{2}$ consumed. We also subtracted the positive control and then added a value of one to make generation activity curves relative to control, where a value of 1 on the generation activity graph is the same as control.

$$
\text { Relative ATP Generation Activity }=\left(\frac{\frac{\int\{N A D H\}_{\text {sample }} \partial t}{\sum\{N A D H\}_{\text {sample }}}+\frac{\int\left\{O_{2}\right\}_{\text {sample }} \partial t}{\sum\left\{O_{2}\right\}_{\text {sample }}}}{2}\right)-\left(\frac{\int\{N A D H\}_{\text {control }} \partial t}{\sum\{N A D H\}_{\text {control }}}+\frac{\int\left\{O_{2}\right\}_{\text {control }} \partial t}{\sum\left\{O_{2}\right\}_{\text {control }}}\right)+1
$$


Bifurcation points relevant to critical signaling events are shown in figure 2.2a for TDZD-8 (40 minutes and 10 hours) and figure $2.2 \mathrm{~b}$ for MEK inh II (20 minutes and 8 hours 20 minutes). Bifurcations were selected by relying on the relative ATP generation activity curves, then relating ATP generation activity curve changes to simultaneous changes in theoretical ATP generation. The first bifurcation is the point before a sustained increase/decrease in ATP generation activity that is simultaneously related to a change in theoretical ATP generation curves for 3 or more doses. The second bifurcation is selected just after there is a rapid decrease/increase in ATP generation activity that is simultaneously related to a change in theoretical ATP generation curves for 3 or more doses. To validate our ATP estimation method, we determined cellular ATP at the 
a

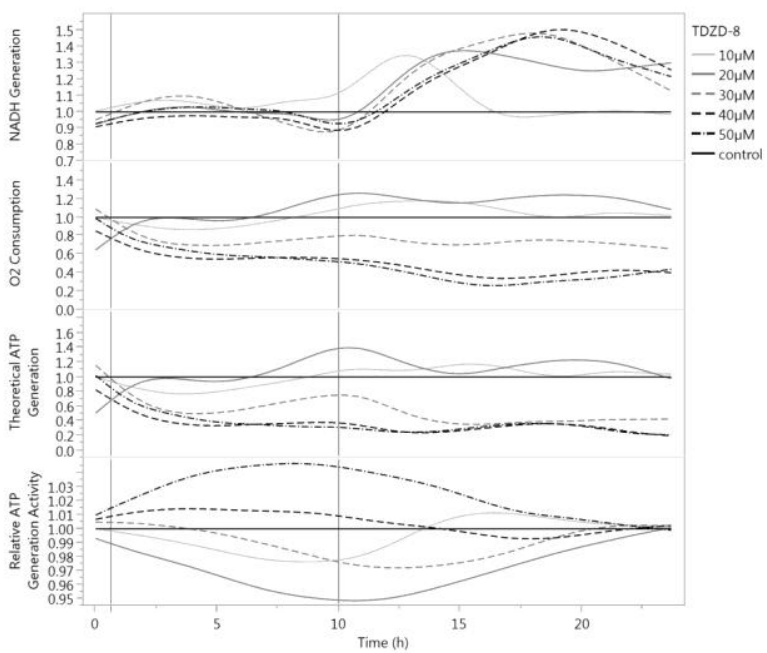

C

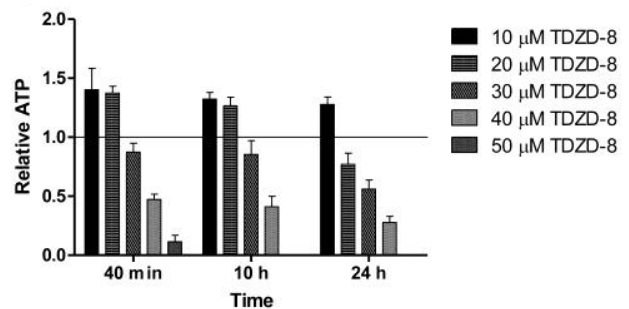

b

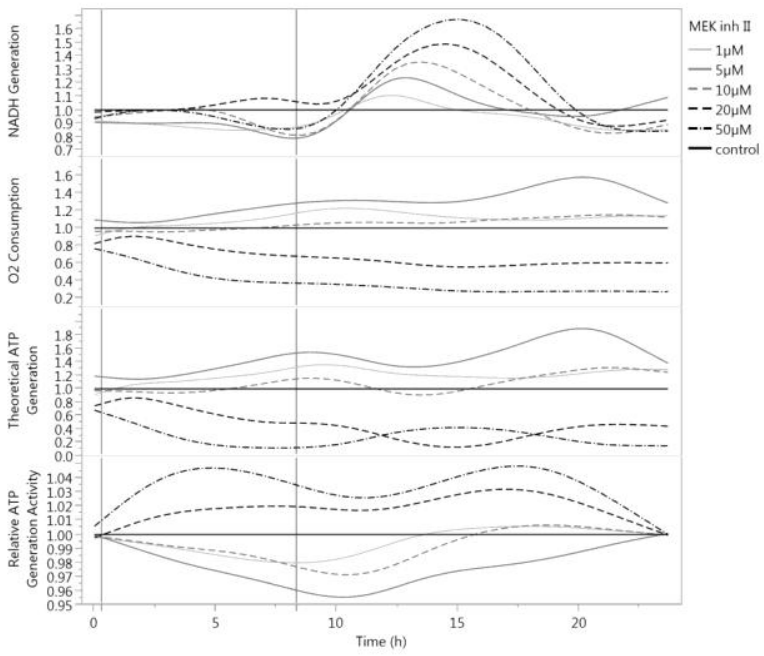

d

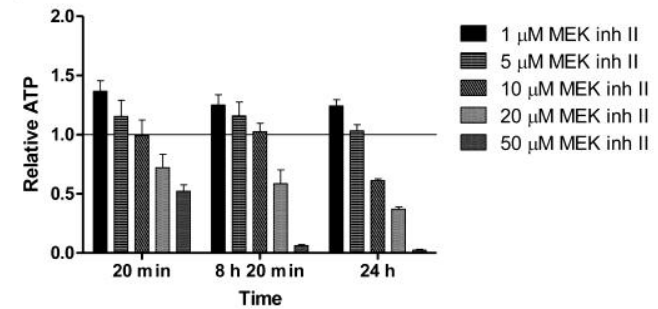

Figure 2.2. Theoretical ATP generation and activity models indicate key bifurcation points for HepG2 cells exposed to TDZD-8 and MEK inh II. a) Using the NADH/NADPH absorbance at $340 \mathrm{~nm}$, NADH generation was measured every 10 minutes for 24 hours following administration of 10, 20, 30, 40, and $50 \mu \mathrm{M}$ doses of TDZD-8 to HepG2 cells. Data is reported as relative NADH generation, which was normalized to controls that received vehicle control ( $<1 \%$ DMSO). Oxygen consumption of HepG2 cells exposed to TDZD-8 was monitored every 10 minutes for 24 hours with an oxygen sensitive probe (MitoXpress). An increase in probe signal indicates an increase in oxygen consumption relative to controls, which received dosing vehicle ( $<1 \%$ DMSO). Theoretical ATP Generation (3rd graph) was calculated using the "If then else loop", as described in Results, which is based on the stoichiometric production of ATP from cellular respiration (oxidative phosphorylation). Relative ATP Generation Activity (4th graph) was calculated from Equation 3. Critical signaling events were selected at $40 \mathrm{~min}$ and $10 \mathrm{~h}$ post-TDZD-8 exposure from our bifurcation analysis described in Results. b) HepG2 cells exposed to 1, 5, 10, 20 and 50 $\mu \mathrm{M}$ doses of MEK inh II were measured and analyzed in the same way as TDZD-8. Critical signaling events were selected at $20 \mathrm{~min}$ and $8 \mathrm{~h} 20 \mathrm{~min}$ post-MEK inh II exposure from our bifurcation analysis described in Results. To determine if the theoretical ATP generation model predicted relative cellular ATP levels, the luciferase assay was used to measure cellular ATP at the time-points of interest. Extracted cellular ATP of HepG2 cells exposed to either c) TDZD-8 or d) MEK inh II were measured using the luciferase assay as described in Materials and Methods. Relative cellular ATP from the luciferase assay of TDZD-8 exposed cells (c) was found to be significantly correlated to our theoretical ATP generation (a, 3rd graph) for TDZD-8 $(r=0.59, P=0.021)$ at the time-points of interest $(40 \mathrm{~min}, 10 \mathrm{~h}$, and $24 \mathrm{~h})$. Relative cellular ATP from the luciferase assay of MEK inh II exposed cells (d) was found to be significantly correlated to the theoretical ATP generation for MEK inh II (b, 3rd graph) for MEK inh II $(r=0.85, P<$ $0.001)$ at the time-points of interest ( $20 \mathrm{~min}, 8 \mathrm{~h} 20 \mathrm{~min}$, and $24 \mathrm{~h}$ ). Results were reported as relative to controls, which received dosing vehicle (<1\% DMSO) and error bars reflect S.E.M. 
time-points of interest for each compound using the luciferase assay (Figure 2.2c,d). Relative cellular ATP from the luciferase assay was found to be significantly correlated to our theoretical ATP generation model for TDZD-8 $(r=0.59, P=0.021)$ (Figure 2.2a, 3rd graph) and MEK inh II $(r=0.85, P<0.001)$ (Figure 2.2b, 3rd graph).

\subsubsection{Multiplex assay to determine phosphorylation of key proteins}

After time points of critical signaling events have been identified, a snapshot of the pharmacodynamic response to xenobiotic exposure can be determined with a highthroughput approach. Inclusion of valuable proteins that cast a wide net on cell death or survival pathways at these time-points are necessary to capture intracellular processes that will determine the cell's eventual fate. In response to cellular stressors (e.g., xenobiotics, endogenous molecules), many kinase pathways converge upon mitochondria, which can result in mitochondrial membrane permeabilization (MMP) mediated death (Kroemer et al., 2007; Rehm et al., 2009). From this, we chose to narrow the list of potential proteins to those that are only a few kinase steps removed from key survival or death proteins that are known to alter mitochondrial activity. Specifically, mitogen-activated protein kinases (MAPKs) have been the subject of intense research for many disease pathologies, especially cancer, due to their role in cell proliferation (Anjum and Blenis, 2008). The EGFR-Ras-Raf-MEK-ERK-RSK signaling network is involved in cellular proliferation, survival, differentiation, and motility (Roberts and Der, 2007); alterations in the normal function of these proteins has been implicated in cancer and other diseases (Sebolt-Leopold and Herrera, 2004). Dysregulated cAMP response 
element binding protein (CREB1) has also been implicated in uncontrolled growth and survival in several cancer cell lines, making it an excellent candidate for this approach (Shukla et al., 2009). JNK and p38 kinases are involved in the coordinated response to external stress stimuli, inflammation and apoptosis (for review, see Pearson et al., 2001), making them viable candidates for this work. Another class of proteins relevant to cell survival and death pathways are heat shock proteins (HSP), specifically HSP27, which has been shown to act as a protective protein, binding to cytochrome c, preventing apoptosis (Bruey et al., 2000). Two proteins intimately involved in mediating stress stimuli signals and cell death are tumor suppressor protein p53 and Akt (Gottlieb et al., 2002). p53-dependent apoptosis plays a critical role regulating pro- and anti-apoptotic factors for transcription, as well as translocating from the cytosol to mitochondria, where it interacts with proteins related to MMP (Sorrentino et al., 2013). Akt on the other hand, has been shown to phosphorylate BCL-2 family proteins, therefore inhibiting MMP (Datta et al., 1997). BAD, another valuable pro-apoptotic BCL-2 family protein, is an excellent candidate protein to monitor for this approach due to its intimate relationship with MMP-related proteins (Zha et al., 1996). To this end, a multiplex bead-based ELISA assay was designed and used to simultaneously determine 19 different protein phosphorylation responses for TDZD-8 and 18 different protein phosphorylation responses for MEK inh II at time points relevant to critical signaling events from ATP production bifurcation points. Cells were lysed at the pre-selected critical signaling time points: 40 minutes and 10 hours post-exposure to TDZD-8 (Figure 2.3) and at 20 minutes 
a

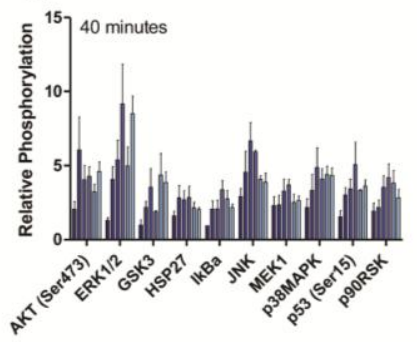

C

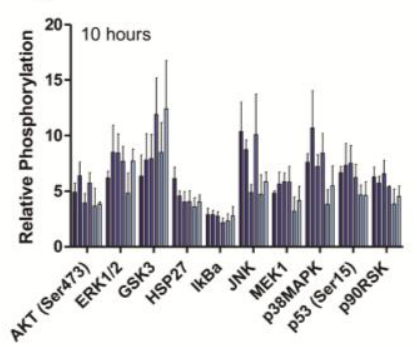

$\mathrm{b}$

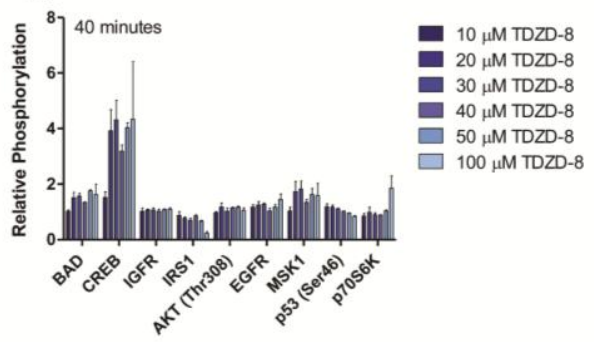

d

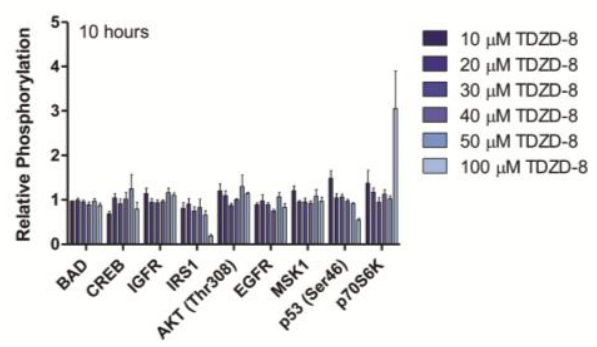

Figure 2.3. Protein phosphorylation responses of HepG2 cells to TDZD-8. From the previously determined temporal bifurcations (40 minutes and 10 hours), relative protein phosphorylation was determined by dosing HepG2 cells with various doses of TDZD-8 (10, 20, 30, 40, 50, and $100 \mu \mathrm{M})$ and lysing the cell membrane at a-b) 40 minutes and c-d) 10 hours post-exposure. Relative phosphorylation was calculated by normalizing to controls, which received vehicle control $(<1 \%$ DMSO). Error bars reflect S.E.M.
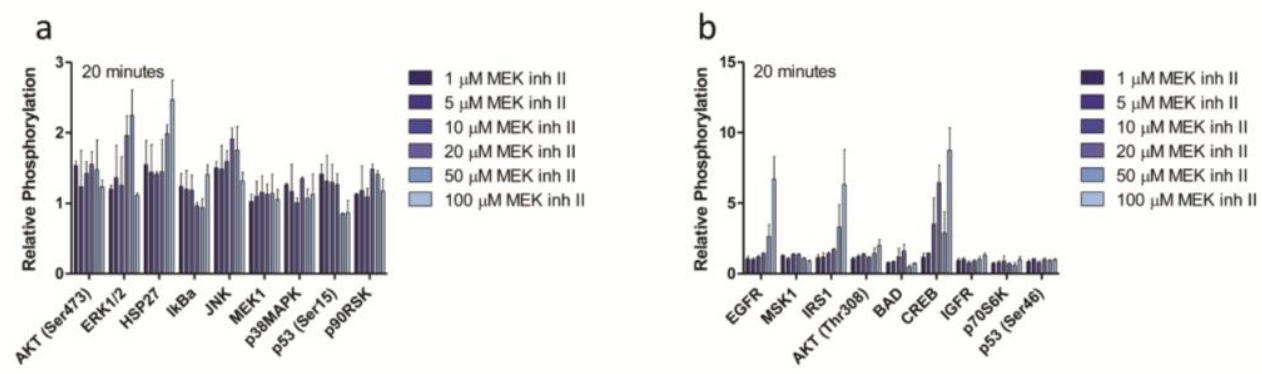

C
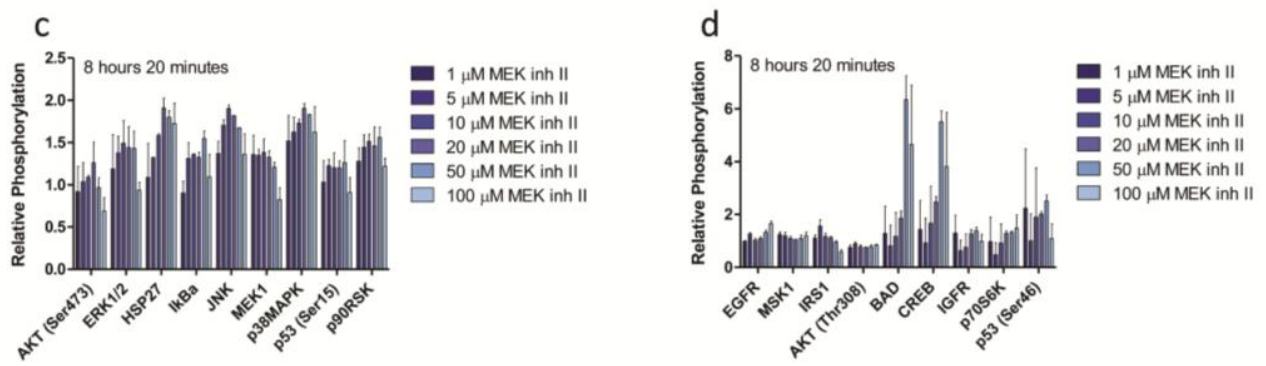

Figure 2.4. Protein phosphorylation responses of HepG2 cells to MEK inh II. From the previously determined temporal bifurcations (20 minutes and 8 hours 20 minutes), relative protein phosphorylation was determined by dosing HepG2 cells with various doses of MEK inh II $(1,5,10$, 20, 50, and $100 \mu \mathrm{M})$ and lysing the cell membrane at a-b) 20 minutes and c-d) 8 hours 20 minutes post-exposure. Relative phosphorylation was calculated by normalizing to controls, which received vehicle control (<1\% DMSO). Error bars reflect S.E.M. 
and 8 hours 20 minutes post-exposure to MEK inh II (Figure 2.4); the phosphoprotein responses of 19 (or 18) different proteins were determined relative to control, which received dosing vehicle (<1\% DMSO).

\subsubsection{Hierarchical cluster analysis}

In order to group protein responses that are relevant to varying doses of TDZD-8 and MEK inh II, we used the unsupervised (i.e., we did not pre-select clusters) Ward twoway Hierarchical clustering method (Ward, 1963) (Figure 2.5a-d). Two-way clustering is necessary to obtain clusters of protein responses across all doses in the first dimension, and dose clusters in the second dimension. Negative control-subtracted raw fluorescence values for cells exposed to TDZD-8 $(10,20,30,40,50$, or $100 \mu \mathrm{M})$ or MEK inh II $(1,5$, $10,20,50$, or $100 \mu \mathrm{M})$, as well as cells exposed to vehicle control only $(0 \mu \mathrm{M})$, were used to determine cluster distances. By including the $0 \mu \mathrm{M}$ phosphoprotein response values, we can include the distance between no dose and the lowest experimentally determined dose to forecast the full plasma membrane degradation dose-response. Additionally, if we assume that an arbitrary/infinite dose of a xenobiotic (i.e., TDZD-8 or MEK inh II) successfully kills all cells exposed, the fluorescence values due to protein phosphorylation should theoretically be zero (via endogenous protein degradation after the plasma membrane is compromised). From this basic theoretical assumption, we included an "infinite dose" column with raw fluorescence values of zero for each protein in the two-way hierarchical cluster analysis. Importantly, the inclusion of a true $0 \mu \mathrm{M}$ dose and a theoretical infinite dose allows for a full dose-response prediction to be made 
without experimentally testing the entire dosing range. As a visual aid, this unsupervised hierarchical clustering method yields a heat map of phosphoprotein responses, where blue represents low response values and red represents high response values. These clusters are based on similarities in phosphoprotein response magnitude profiles. After performing two-way hierarchical cluster analysis on each protein for each set of replicates (clusters not shown, only averaged responses are depicted in Figures 2.5a-d), cluster distances between doses were used for dose-response predictions.

\subsubsection{Forecasting dose-response from hierarchical cluster analysis}

By monitoring the phosphorylation response of proteins involved in cellular survival and/or death pathways at early time-points, the fate of the cell may be forecasted. After determining the cluster distances from two-way hierarchical cluster analysis with Ward's minimum variance, these values were used to forecast the relative plasma membrane degradation dose-response of TDZD-8 (Figures 2.5 e,f) and MEK inh II exposed HepG2 cells (Figures $2.5 \mathrm{~g}, \mathrm{~h}$ ). To forecast the dose-response, the cluster distance between doses including $0 \mu \mathrm{M}$ (vehicle control) and the theoretical infinite dose were organized in order of increasing dose. These values were then integrated across each dose (x) interval and divided by the sum of all dose cluster distances, as described by equation 4:

$$
\frac{\int\{\text { cluster distance }\} \partial x}{\sum_{0}^{\text {inf dose }}\{\text { cluster distance }\}}
$$


a
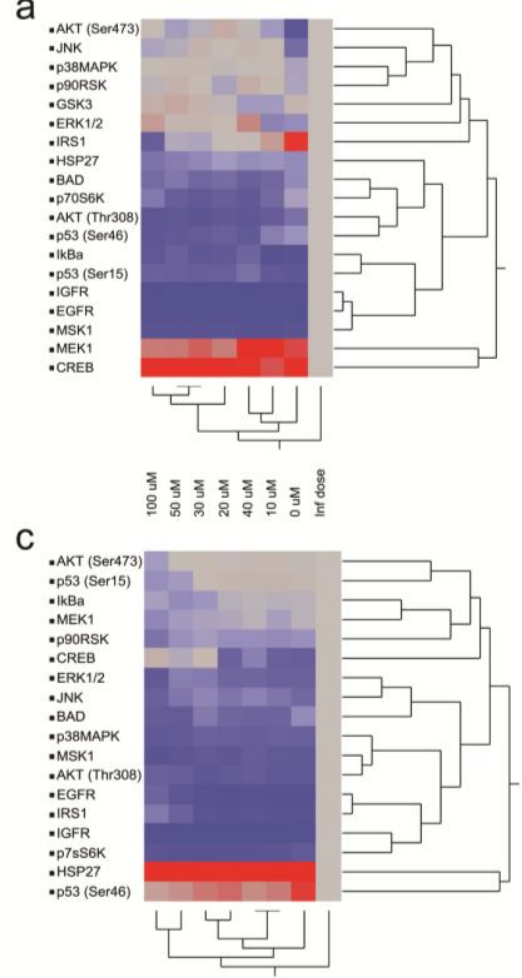

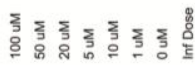

b
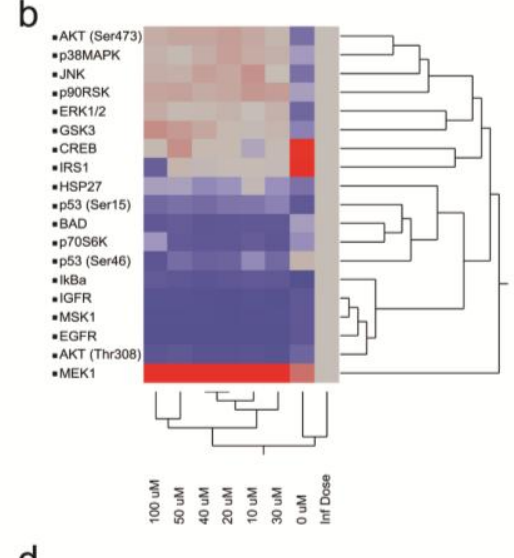

d

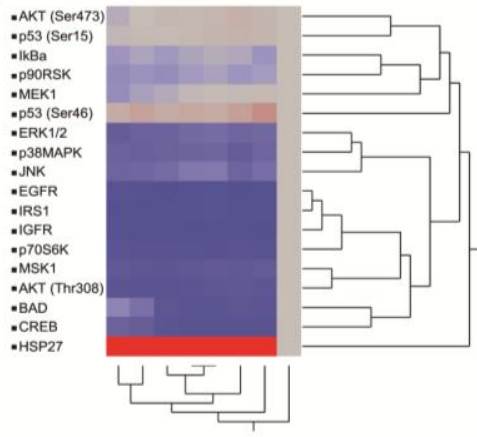

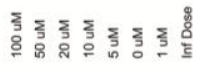

e

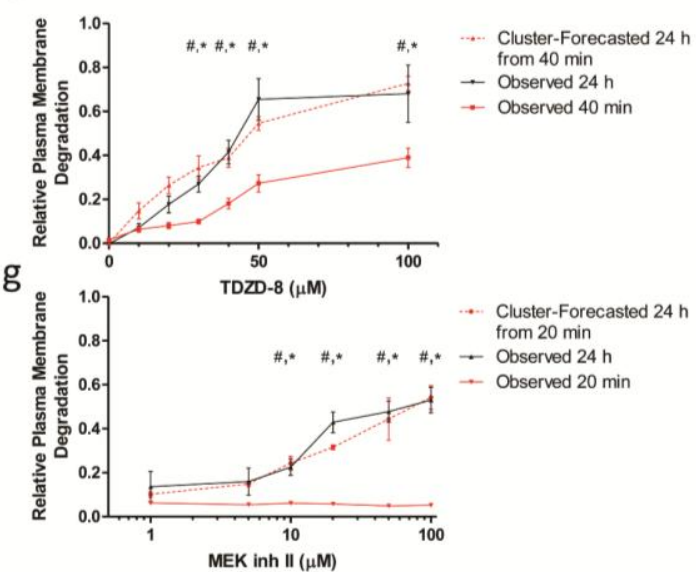

f

$\mathrm{h}$

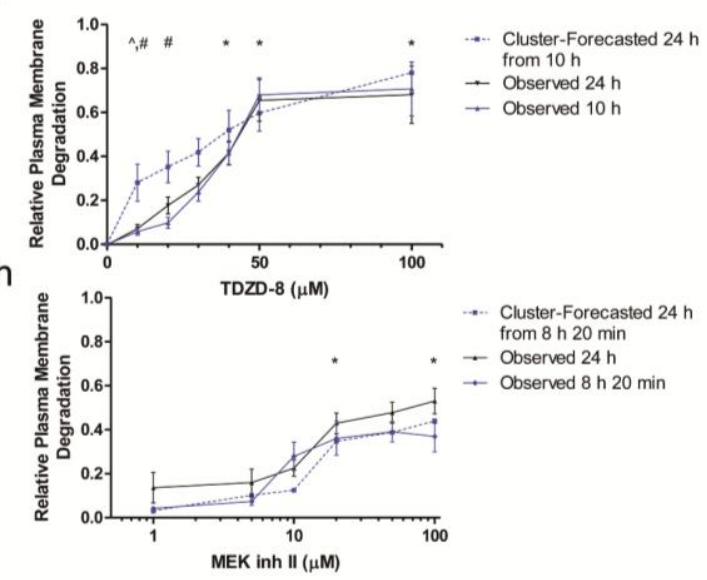

Figure 2.5. Forecasting plasma membrane degradation from two-way hierarchical cluster analysis distances. The phosphoprotein fluorescence responses of HepG2 cells to various doses of TDZD-8 (10, 20, $30,40,50$, and $100 \mu \mathrm{M})$ or MEK inh II $(1,5,10,20,50$, and $100 \mu \mathrm{M})$ were analyzed with the unsupervised Ward two-way hierarchical clustering method. Each set of replicates were treated as a different cluster for our analyses; however, to save space, we will show the averaged fluorescence values at each time point. Shown above, cluster analyss of phosphoprotein responses to: a) TDZD-8 at 20 minutes post-exposure, b) TDZD-8 at 10 hours post-exposure, c) MEK inh II at 20 minutes post-exposure, and d) MEK inh II at 8 hours 20 minutes post-exposure. After the cluster analyses were performed, the cluster distances associated with each dose are assembled and integrated across the dosing range to formulate the forecasted relative plasma membrane degradation dose-response curve (e-h). E) Experimentally observed relative plasma 
membrane responses to TDZD-8 at 40 minutes and 24 hours post-exposure are connected with red and black solid lines, respectively. The forecasted 24 hour plasma membrane degradation responses that were calculated with cluster distances from 40 minutes post-TDZD-8 exposure phosphoprotein responses are connected with a red dashed line. F) Experimentally observed relative plasma membrane degradation responses to TDZD-8 at 10 hours and 24 hours post-exposure are connected with blue and black solid lines, respectively. The forecasted 24 hour plasma membrane degradation responses that were calculated with cluster distances from 10 hours post-TDZD-8 exposure phosphoprotein responses are connected with a blue dashed line. G) Experimentally observed relative plasma membrane degradation responses to MEK inh II at 20 minutes and 24 hours post-exposure are connected with red and black solid lines, respectively. The forecasted 24 hour plasma membrane degradation responses that were calculated with cluster distances from 20 minutes post-MEK inh II exposure phosphoprotein responses are connected with a red dashed line. $\mathrm{H}$ ) Experimentally observed relative plasma membrane degradation responses to MEK inh II at 8 hours 20 minutes and 24 hours post-exposure are connected with blue and black solid lines, respectively. The forecasted 24 hour plasma membrane degradation responses that were calculated with cluster distances from 8 hours 20 minutes post-exposure phosphoprotein responses are connected with a blue dashed line. For graphs in figures e-h, relative plasma membrane responses were analyzed using two-way ANOVA. Clusterforecasted responses found to be significantly different $(P<0.05)$ from observed 24 hour responses are marked with $\wedge$, cluster-forecasted responses found to be significantly different $(P<0.05)$ from early timepoint observed responses are marked with \#, and observed early time-point responses found to be significantly different $(P<0.05)$ from observed 24 hour responses are marked with *. Error bars reflect \pm S.E.M.

To determine if the forecasted dose-response (from early time points to TDZD-8 or MEK inh II exposure) matched the observed 24 hour plasma membrane dose response, a twoway ANOVA was performed. The 24 hour observed and forecasted (from 40 minutes post-TDZD- 8 exposure and 20 minutes and 8 hours 20 minutes post-MEK inh II exposure) plasma membrane responses were not significantly different at any dose. The 24 hour observed and forecasted plasma membrane degradation at 10 hours post-TDZD-8 exposure were only statistically different at the $10 \mu \mathrm{M}$ TDZD-8 dose $(P<0.05)$. Additionally, observed vs. forecasted plasma membrane degradation at the early time points (20 minutes plasma membrane degradation post-MEK inh II exposure observed vs. forecasted and 40 minutes plasma membrane degradation post-TDZD-8 exposure observed vs. forecasted) were significantly different $(P<0.05)$ from each other at 30,40, 50 and $100 \mu \mathrm{M}$ TDZD-8 and 10, 20, 50, and $100 \mu \mathrm{M}$ MEK inh II indicating that we are 
not measuring plasma membrane degradation at those early time points, rather we are capturing early critical signaling events related to the eventual cellular fate.

\subsection{Discussion}

Understanding the cell's own pharmacodynamic interpretation of exposure to a potentially toxic xenobiotic or therapeutic is imperative to determining the eventual cellular fate. Before morphological changes (e.g., plasma membrane degradation) indicate the overall cellular state, the intracellular domain is coordinating a dynamic and networked response that dictates the eventual cellular outcome. By exploiting critical pharmacodynamic events related to the eventual cellular fate decision, the cellular response to external stimuli (e.g., xenobiotic exposure or general stress) can be forecasted from significantly early time points post-exposure. In this paper, human hepatocellular carcinoma derived HepG2 cells were exposed to two disparate inhibitors, TDZD-8 (GSK$3 \beta$ substrate-competitive inhibitor) and MEK inh II (MEK1 inhibitor), to test an approach that combines several datasets to forecast the eventual cellular outcome. The datasets used in this work incorporated theoretical ATP generation for the identification of critical signaling bifurcation points, followed by determinations of the phosphoprotein response at these time-points post-exposure for further cluster analyses to forecast the plasma membrane degradation dose-response at 24 hours of exposure. This concept has a universal approach: utilizing the cell's own early responses post-exposure to forecast the cell's eventual fate that may be irrespective of the xenobiotic's intracellular mode(s) of action. 
To our knowledge, reliable in vitro approaches that are capable of determining important intracellular processes in real-time, such as ATP production, without perturbing native intracellular biochemical processes have yet to be determined (Imamura et al., 2009). Real-time oxygen consumption has been used to identify temporal events post-xenobiotic exposure that are related to intracellular signaling, due to the relationship between oxygen consumption and oxidative phosphorylation (Janssen-Heininger et al., 2008). While we have had some success using oxygen consumption alone to select critical signaling events (Boyd et al., 2013), this method can be improved by exploiting the intricate intracellular relationship between NADH generation, oxygen consumption, and ATP production. By extracellularly monitoring analogs of ATP production over time after xenobiotic exposure, critical signaling events may be estimated without potentially disrupting intracellular activity. In this manner, two bifurcation time points were identified for HepG2 cells post-TDZD-8 or MEK inh II exposure. Since kinase signaling, which utilizes appreciable amounts of available ATP, is an energy demanding process (Hammerman et al., 2004), these early bifurcation points may be indicative of critical signaling processes relevant to intracellular-level decision making for the cell's overall eventual fate. This approach assumes that all oxygen consumption and NADH generation measured in vitro is attributed to oxidative phosphorylation for ATP synthesis. Thus, this model does not incorporate oxidative phosphorylation-independent oxygen consumption, which can vary based on cell type (Herst and Berridge, 2007; Trimarchi et al., 2000). Additionally, cellular glucose, calcium, and magnesium were not measured in this study. For future model development, these critical cellular components may be measured to more accurately select key time-points. This new approach may translate well into other 
cell lines with similar amounts of mitochondria, such as heart and kidney (Veltri et al., 1990), or similar mitochondrial bioenergetics, such as the heart, testes, and brain (Menzies and Gold, 1971). To fully validate this method, additional cell lines, both immortalized and primary, in various tissues need to be tested.

Cell death can take a number of routes, such as necrosis, apoptosis, or autophagy, to name a few (for review, see Galluzzi et al., 2012), whose time course after initial exposure can last anywhere from minutes to days (Lemasters et al., 1998). Irrespective of the cell death modality, the switch between cell death and survival revolves around the integration of signals at mitochondrial membranes that contribute to mitochondrial membrane permeability (MMP) (Kroemer et al., 2007). Since MMP is a major player in the eventual cellular fate decision of survival or death, monitoring protein signaling cascades that converge upon mitochondrial membranes is an advantageous approach. Forecasting a cell's eventual fate can be accomplished by monitoring the phosphorylation status of proteins that are relevant to survival and death pathways at critical signaling time-points following exposure to xenobiotics. While the minimum number of proteins necessary to forecast future cell death has yet to be determined, our proof of principle study included 19 judiciously selected phosphoproteins in a multiplexable and highthroughput bead-based ELISA assay. In this work, we chose to cast a wide net on cell death and survival pathways at pre-selected time-points to capture critical processes responsible for determining the cell's eventual fate. The proteins selected for this study 

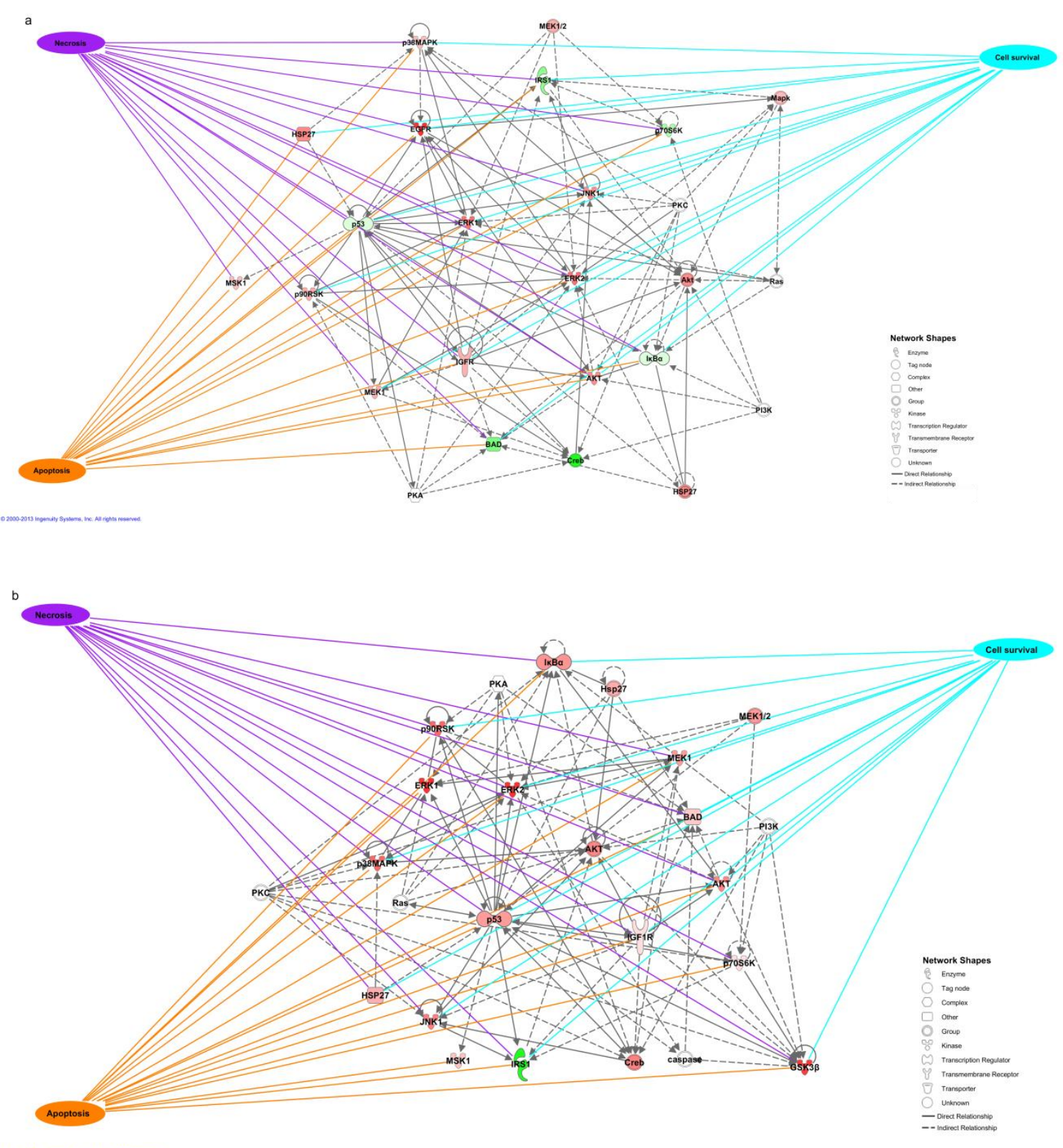

Figure 2.6. Ingenuity pathway analysis of phosphoprotein responses. The most significant network interactions from the 19 different phosphoproteins measured and important intermediate proteins were compiled in IPA. Since the proteins included in the analyses did not change for all doses at both time points, only one dataset per inhibitor is shown in the figure. As examples, a) $50 \mu \mathrm{M}$ MEK inh II at 20 minutes post-exposure and b) $50 \mu \mathrm{M}$ TDZD- 8 at 40 minutes post-exposure are included in the figure above. Normalized phosphoproteins that were greater than control are shown in red and phosphoproteins that were less than control are shown in green. IPA analysis indicated that the most significant molecular and cellular function of both inhibitor datasets were cell death and survival; specifically, apoptosis, cell survival, and necrosis were the most significant biological functions of the proteins included in the analyses. 
were confirmed to be most relevant to cell death (specifically apoptosis and necrosis) and survival by using Ingenuity Pathway Analysis (IPA)-constructed multidirectional interaction networks with biological function overlay (Figure 2.6a and b) to visualize the connectivity of the proteins and their association with various biological functions. For future development of this approach, additional phosphoproteins or different combinations of phosphoproteins should be measured to determine the optimal number and type of protein to forecast eventual cellular fate. Additionally, this approach only monitored phosphoprotein responses to xenobiotic exposure. However, different types of post-translational modifications, such as glycosylation, methylation, or acetylation, may also be useful for this type of methodology.

While relative phosphoprotein responses of HepG2 cells to various doses of inhibitors at key time points post-exposure offer a wealth of information about these critical signaling events, a more digestible data platform and data transformation are required to construct the forecasted dose-response to TDZD-8 and MEK inh II exposure. Hierarchical cluster analysis using Ward's method produces clusters that minimize the variance within each cluster (Ward, 1963). Going a step further, two-way cluster analysis allows for the simultaneous clustering of both variables in this paper (i.e., phosphoprotein response profiles and dose). In the first dimension, the protein responses across all doses $(0 \mu \mathrm{M}-100 \mu \mathrm{M}$ and theoretical infinite dose $)$ are clustered to minimize the within-cluster variance and simultaneously in the second dimension, doses with similar phosphoprotein response profiles are clustered together. It is the latter dimension that provides useful distances based on the within-group minimized ANOVA sum of squares across all clusters. By including the vehicle control phosphoprotein responses $\left(\begin{array}{lll}0 & \mu \mathrm{M}\end{array}\right)$ and 
theoretical infinite dose phosphoprotein responses, the forecasted dose-response range can be computed, regardless of the actual full dose-response curve (i.e., this circumvents the need to pre-determine exposure doses that go from $0 \%$ cell death to $100 \%$ cell death). Each replicate dose cluster's ANOVA sum of squares distances were placed in order of increasing dose $(0 \mu \mathrm{M}$ - theoretical infinite dose $)$ and integrated across the dosing range to formulate the forecasted relative plasma membrane degradation doseresponse curve. While this method does not pinpoint the full intracellular mode(s) of action for the two inhibitors included in this study, the 24 hour relative plasma membrane degradation dose-response was accurately forecasted as early as 20 minutes postexposure to MEK inh II and 40 minutes post-exposure to TDZD-8 exposure. Additionally, the observed relative plasma membrane degradation at 20 minutes postexposure to MEK inh II and 40 minutes post-exposure to TDZD-8 has little to no death occurring. Therefore, the phosphoprotein responses at 20 minutes and 40 minutes postexposure are likely associated with the cell's own pharmacodynamic interpretation of exposure and eventual cell fate decision, as opposed to actual cell death occurring at this time point.

In conclusion, this approach offers several advantages over current methodologies to determine future xenobiotic toxicity: using extracellular techniques that do not disrupt the native intracellular biochemical processes to estimate ATP production relevant to critical signaling events, selecting phosphoproteins relevant to survival and/or death pathways at critical signaling time-points that may be predictive of the cell's eventual fate (i.e., 24 hours post-exposure), and using hierarchical cluster analysis to predict the 24 hour relative plasma membrane degradation from early phosphoprotein responses. This 
method has been shown to accurately forecast the eventual cellular fate of HepG2 cells exposed to two different xenobiotics, with disparate intracellular modes of action, and may theoretically work for any intracellular mode of toxicity or cell death modality. Finally, our results for this approach suggest that an analysis aggregating several different protein networks may be more informative of in vitro toxicity than any individual pathways or biomarkers. This has broad implications for many scientific fields, including toxicology and pharmacology. These advantages allow for future studies in a highthroughput framework to quickly assess and forecast the eventual xenobiotic toxicity of cells in vitro. Additionally, the ability to identify early critical signaling events postexposure that are related to important cellular endpoints, such as survival or death, may significantly impact the pharmacological understanding of the therapeutic window for treatment strategies, and potentially alter the forecasted cellular fate by probing the endogenous signaling responses to treatments at these time points.

\subsection{References}

Ainscow, E.K., Brand, M.D., 1999. Top-down control analysis of ATP turnover, glycolysis and oxidative phosphorylation in rat hepatocytes. Eur J Biochem 263, 671-685.

Altekruse, S.F., McGlynn, K.A., Reichman, M.E., 2009. Hepatocellular carcinoma incidence, mortality, and survival trends in the United States from 1975 to 2005. J Clin Oncol 27, 1485-1491.

Anjum, R., Blenis, J., 2008. The RSK family of kinases: emerging roles in cellular signalling. Nat Rev Mol Cell Biol 9, 747-758. 
Bakare, O., Ashendel, C.L., Peng, H., Zalkow, L.H., Burgess, E.M., 2003. Synthesis and MEK1 inhibitory activities of imido-substituted 2-chloro-1,4-naphthoquinones. Bioorg Med Chem 11, 3165-3170.

Ballif, B.A., Blenis, J., 2001. Molecular mechanisms mediating mammalian mitogenactivated protein kinase (MAPK). Cell Growth Differ 12, 397-408.

Boyd, J., Vrana, J.A., Williams, H.N., 2013. In vitro approach to predict posttranslational phosphorylation response to mixtures. Toxicology 313, 113-121.

Bruey, J.M., Ducasse, C., Bonniaud, P., Ravagnan, L., Susin, S.A., Diaz-Latoud, C., Gurbuxani, S., Arrigo, A.P., Kroemer, G., Solary, E., Garrido, C., 2000. Hsp27 negatively regulates cell death by interacting with cytochrome c. Nat Cell Biol 2 , 645-652.

Buttgereit, F., Brand, M.D., 1995. A hierarchy of ATP-consuming processes in mammalian cells. Biochem J 312 ( Pt 1), 163-167.

Datta, S.R., Dudek, H., Tao, X., Masters, S., Fu, H., Gotoh, Y., Greenberg, M.E., 1997. Akt phosphorylation of BAD couples survival signals to the cell-intrinsic death. Cell 91, 231-241.

Eguchi, Y., Shimizu, S., Tsujimoto, Y., 1997. Intracellular ATP levels determine cell death fate by apoptosis or necrosis. Cancer Res 57, 1835-1840.

Eguchi, Y., Srinivasan, A., Tomaselli, K.J., Shimizu, S., Tsujimoto, Y., 1999. ATPdependent steps in apoptotic signal transduction. Cancer Res 59, 2174-2181.

Galluzzi, L., Vitale, I., Abrams, J.M., Alnemri, E.S., Baehrecke, E.H., Blagosklonny, M.V., Dawson, T.M., Dawson, V.L., El-Deiry, W.S., Fulda, S., Gottlieb, E., Green, D.R., Hengartner, M.O., Kepp, O., Knight, R.A., Kumar, S., Lipton, S.A., Lu, X., Madeo, F., Malorni, W., Mehlen, P., Nunez, G., Peter, M.E., Piacentini, M., Rubinsztein, D.C., Shi, Y., Simon, H.U., Vandenabeele, P., White, E., Yuan, J., Zhivotovsky, B., Melino, G., Kroemer, G., 2012. Molecular definitions of cell death subroutines: recommendations of the. Cell Death Differ 19, 107-120.

Gottlieb, T.M., Leal, J.F., Seger, R., Taya, Y., Oren, M., 2002. Cross-talk between Akt, p53 and Mdm2: possible implications for the regulation of. Oncogene 21, 12991303. 
Hammerman, P.S., Fox, C.J., Thompson, C.B., 2004. Beginnings of a signal-transduction pathway for bioenergetic control of cell survival. Trends Biochem Sci 29, 586592.

Herst, P.M., Berridge, M.V., 2007. Cell surface oxygen consumption: a major contributor to cellular oxygen consumption in glycolytic cancer cell lines. Biochim Biophys Acta 1767, 170-177.

Huynh, H., Nguyen, T.T., Chow, K.H., Tan, P.H., Soo, K.C., Tran, E., 2003. Overexpression of the mitogen-activated protein kinase (MAPK) kinase (MEK)MAPK. BMC Gastroenterol 3, 19.

Imamura, H., Nhat, K.P., Togawa, H., Saito, K., Iino, R., Kato-Yamada, Y., Nagai, T., Noji, H., 2009. Visualization of ATP levels inside single living cells with fluorescence resonance energy transfer-based genetically encoded indicators. Proc Natl Acad Sci U S A 106, 15651-15656.

Janssen-Heininger, Y.M., Mossman, B.T., Heintz, N.H., Forman, H.J., Kalyanaraman, B., Finkel, T., Stamler, J.S., Rhee, S.G., van der Vliet, A., 2008. Redox-based regulation of signal transduction: principles, pitfalls, and promises. Free Radic Biol Med 45, 1-17.

Kadenbach, B., 1986. Regulation of respiration and ATP synthesis in higher organisms: hypothesis. J Bioenerg Biomembr 18, 39-54.

Kanzawa, T., Germano, I.M., Komata, T., Ito, H., Kondo, Y., Kondo, S., 2004. Role of autophagy in temozolomide-induced cytotoxicity for malignant glioma cells. Cell Death Differ 11, 448-457.

Kholodenko, B.N., 2006. Cell-signalling dynamics in time and space. Nat Rev Mol Cell Biol 7, 165-176.

Kim, L., Kimmel, A.R., 2000. GSK3, a master switch regulating cell-fate specification and tumorigenesis. Curr Opin Genet Dev 10, 508-514.

Kondo, Y., Kanzawa, T., Sawaya, R., Kondo, S., 2005. The role of autophagy in cancer development and response to therapy. Nat Rev Cancer 5, 726-734. 
Kroemer, G., Galluzzi, L., Brenner, C., 2007. Mitochondrial membrane permeabilization in cell death. Physiol Rev 87, 99-163.

Kumar, D., Srikanth, R., Ahlfors, H., Lahesmaa, R., Rao, K.V., 2007. Capturing cell-fate decisions from the molecular signatures of a. Mol Syst Biol 3, 150.

Leist, M., Single, B., Castoldi, A.F., Kuhnle, S., Nicotera, P., 1997. Intracellular adenosine triphosphate (ATP) concentration: a switch in the decision between apoptosis and necrosis. J Exp Med 185, 1481-1486.

Lemasters, J.J., Nieminen, A.L., Qian, T., Trost, L.C., Elmore, S.P., Nishimura, Y., Crowe, R.A., Cascio, W.E., Bradham, C.A., Brenner, D.A., Herman, B., 1998. The mitochondrial permeability transition in cell death: a common mechanism in necrosis, apoptosis and autophagy. Biochim Biophys Acta 1366, 177-196.

Madoz-Gurpide, J., Canamero, M., Sanchez, L., Solano, J., Alfonso, P., Casal, J.I., 2007. A proteomics analysis of cell signaling alterations in colorectal cancer. Mol Cell Proteomics 6, 2150-2164.

Martinez, A., Alonso, M., Castro, A., Perez, C., Moreno, F.J., 2002. First non-ATP competitive glycogen synthase kinase 3 beta (GSK-3beta) inhibitors: thiadiazolidinones (TDZD) as potential drugs for the treatment of Alzheimer's disease. J Med Chem 45, 1292-1299.

Menzies, R.A., Gold, P.H., 1971. The turnover of mitochondria in a variety of tissues of young adult and aged rats. J Biol Chem 246, 2425-2429.

Messam, C.A., Pittman, R.N., 1998. Asynchrony and commitment to die during apoptosis. Exp Cell Res 238, 389-398.

Mitsui, H., Takuwa, N., Maruyama, T., Maekawa, H., Hirayama, M., Sawatari, T., Hashimoto, N., Takuwa, Y., Kimura, S., 2001. The MEK1-ERK map kinase pathway and the PI 3-kinase-Akt pathway independently. Int J Cancer 92, 55-62.

Nelson, D.L., Cox, M.M., 2008. Lehninger Principles of Biochemistry. W. H. Freeman.

Newman, R.H., Hu, J., Rho, H.S., Xie, Z., Woodard, C., Neiswinger, J., Cooper, C., Shirley, M., Clark, H.M., Hu, S., Hwang, W., Seop Jeong, J., Wu, G., Lin, J., Gao, X., Ni, Q., Goel, R., Xia, S., Ji, H., Dalby, K.N., Birnbaum, M.J., Cole, P.A., 
Knapp, S., Ryazanov, A.G., Zack, D.J., Blackshaw, S., Pawson, T., Gingras, A.C., Desiderio, S., Pandey, A., Turk, B.E., Zhang, J., Zhu, H., Qian, J., 2013.

Construction of human activity-based phosphorylation networks. Mol Syst Biol 9, 655.

Pearson, G., Robinson, F., Beers Gibson, T., Xu, B.E., Karandikar, M., Berman, K., Cobb, M.H., 2001. Mitogen-activated protein (MAP) kinase pathways: regulation and physiological. Endocr Rev 22, 153-183.

Rehm, M., Huber, H.J., Hellwig, C.T., Anguissola, S., Dussmann, H., Prehn, J.H., 2009. Dynamics of outer mitochondrial membrane permeabilization during apoptosis. Cell Death Differ 16, 613-623.

Roberts, P.J., Der, C.J., 2007. Targeting the Raf-MEK-ERK mitogen-activated protein kinase cascade for the. Oncogene 26, 3291-3310.

Schilling, M., Maiwald, T., Hengl, S., Winter, D., Kreutz, C., Kolch, W., Lehmann, W.D., Timmer, J., Klingmuller, U., 2009. Theoretical and experimental analysis links isoform-specific ERK signalling to. Mol Syst Biol 5, 334.

Sebolt-Leopold, J.S., Herrera, R., 2004. Targeting the mitogen-activated protein kinase cascade to treat cancer. Nat Rev Cancer 4, 937-947.

Shukla, A., Bosenberg, M.W., MacPherson, M.B., Butnor, K.J., Heintz, N.H., Pass, H.I., Carbone, M., Testa, J.R., Mossman, B.T., 2009. Activated cAMP response element binding protein is overexpressed in human. Am J Pathol 175, 2197-2206.

Sorrentino, G., Mioni, M., Giorgi, C., Ruggeri, N., Pinton, P., Moll, U., Mantovani, F., Del Sal, G., 2013. The prolyl-isomerase Pin1 activates the mitochondrial death program of p53. Cell Death Differ 20, 198-208.

Trimarchi, J.R., Liu, L., Porterfield, D.M., Smith, P.J., Keefe, D.L., 2000. Oxidative phosphorylation-dependent and -independent oxygen consumption by individual preimplantation mouse embryos. Biol Reprod 62, 1866-1874.

Vander Heiden, M.G., Cantley, L.C., Thompson, C.B., 2009. Understanding the Warburg effect: the metabolic requirements of cell proliferation. Science 324, 1029-1033. 
Veltri, K.L., Espiritu, M., Singh, G., 1990. Distinct genomic copy number in mitochondria of different mammalian organs. J Cell Physiol 143, 160-164.

Wang, Q., Zhou, Y., Wang, X., Evers, B.M., 2006. Glycogen synthase kinase-3 is a negative regulator of extracellular signal-regulated kinase. Oncogene 25, 43-50.

Warburg, O., 1956. On the origin of cancer cells. Science 123, 309-314.

Ward, J.H., 1963. HIERARCHICAL GROUPING TO OPTIMIZE AN OBJECTIVE FUNCTION. Journal of the American Statistical Association 58, 236-\&.

Yamamoto, A., Tagawa, Y., Yoshimori, T., Moriyama, Y., Masaki, R., Tashiro, Y., 1998. Bafilomycin A1 prevents maturation of autophagic vacuoles by inhibiting fusion between autophagosomes and lysosomes in rat hepatoma cell line, H-4-II-E cells. Cell Struct Funct 23, 33-42.

Yang, N.C., Ho, W.M., Chen, Y.H., Hu, M.L., 2002. A convenient one-step extraction of cellular ATP using boiling water for the luciferin-luciferase assay of ATP. Anal Biochem 306, 323-327.

Zha, J., Harada, H., Yang, E., Jockel, J., Korsmeyer, S.J., 1996. Serine phosphorylation of death agonist BAD in response to survival factor. Cell 87, 619-628. 
Chapter 3

Forecasting cell death dose-response to mixtures in vitro 


\section{Forecasting cell death dose-response to mixtures in vitro ${ }^{I}$}

Individuals are continuously exposed to complex chemical mixtures, whether they are derived from environmental or pharmaceutical (polypharmacy) exposures. While this is an everyday reality, most chemical risk assessments are carried out on a single chemical, or single-dose binary mixtures. Understanding and ultimately predicting chemical mixture effects could not only elucidate potential risk factors associated with exposures, but also offer the opportunity to develop a greater understanding of cellular mechanisms of environmental exposure and even drug discovery. Previously, we have shown that post-translational phosphorylation responses to chemical mixture exposures can be predicted in vitro using Bliss Independence (Boyd et al., 2013). We have also shown that $24 \mathrm{~h}$ in vitro cell death dose-response can be forecasted at significantly earlier time-points (as early as $20 \mathrm{~min}$ or $40 \mathrm{~min}$, depending upon the exposure) by using twoway cluster analysis of phosphorylation responses (Vrana et al., 2014). However, if both methodologies are combined, it may be possible to forecast $24 \mathrm{~h}$ mixture cytotoxicity without measuring the mixture itself; ultimately representing a model capable of predicting mixture toxicity using only individual compound phosphoprotein responses of key kinases at critical signaling events. To this end, we have combined the two methodologies to potentially forecast mixture cell death dose-response using Bliss Independence-predicted phosphorylation values and compared those results to observedforecasted and observed values. To fully test this method, we used two different mixtures: Deguelin and KCN (both ETC inhibitors) and MEK inh II and TDZD-8

\footnotetext{
${ }^{1}$ Parts of this chapter have been published previously from Boyd, J., Vrana, J.A., Williams, H.N. (2013) In vitro approach to predict post-translational phosphorylation response to mixtures. Toxicology 313, 113-21. Reproduced with permission from Elsevier.
} 
(disparate kinase inhibitors). The phosphoprotein targets used in this study are key kinases involved in cellular survival and/or death pathways. By monitoring the response of these phosphoproteins at critical signaling time-points, the eventual cellular fate may be forecasted. Additionally, by using Bliss Independence-predicted phosphoprotein responses for two-way cluster analyses, cytotoxicity in response to a potential mixture may be forecasted without experimentally measuring the mixture.

\subsection{Introduction}

Humans are continuously exposed to complex chemical mixtures, from the air we breathe and the water we drink to the medications we take daily. While this is an everyday reality, most chemical risk assessments are carried out on a single chemical, or single-dose binary mixtures (Cedergreen et al., 2008). Traditional risk assessment testing techniques involve screening potential agents with a single high dose exposure, using a suite of in vivo animal models and apical endpoint analyses, such as neurotoxicity or developmental toxicity, and narrowly-focused mode of action analysis, such as specific cytotoxicity or mutagenicity assays (Dix et al., 2007). This low-throughput approach that relies heavily on animal testing and high dose exposure analyses has created an enormous backlog of chemicals waiting to be evaluated for potential toxicity (Bushnell et al., 2010; Bhattacharya et al., 2011). This risk assessment crisis prompted the 2007 U.S. National Research Council (NRC) report, Toxicity Testing in the 21st Century: A Vision and a Strategy (NRC, 2007). In this report, the NRC proposed a paradigm shift from lowthroughput in vivo studies to new risk assessment strategies that utilizes high-throughput 
in vitro screening assays to exploit early cellular changes (such as signaling pathway perturbations and alterations in cellular bioenergetics) to reveal mechanistic information about adverse or adaptive effects after xenobiotic exposure (Hartung, 2009; Krewski et al., 2010; Attene-Ramos et al., 2013). With this mechanistic information, better risk assessment models can be constructed for single chemical and mixture exposure in humans, greatly benefiting the public health sector.

New risk assessment approaches would include a suite of assays that cast a wide net on early cellular changes, such as changes in cellular bioenergetics, and various pathway perturbations, such as alterations in post-translational modifications (PTMs) post-exposure, to fully understand the cellular response, whether that be adaptation after exposure to a new homeostatic state, or cell death (Andersen and Krewski, 2009). Understanding and eventually predicting chemical mixture effects could not only elucidate potential risk factors associated with exposures, but also offers the opportunity to estimate mixtures toxicity at various doses without measuring the actual mixture. With this new risk assessment methodology in mind, we have shown that early pathway perturbation responses, such as phosphorylation PTMs, to chemical mixture exposures (electron transport chain (ETC) inhibitors as well as broad kinase inhibitors) can be predicted in vitro using Bliss Independence (with accuracy > $77 \%$ ) (Boyd et al., 2013). We have also shown that $24 \mathrm{~h}$ in vitro cell death dose-response can be forecasted at early time points (as early as 20 min or $40 \mathrm{~min}$, depending upon the exposure) by using twoway cluster analysis of phosphorylation responses at time points relevant to changes in cellular bioenergetics and, consequently, perturbations in signal transduction (Vrana et $a l ., 2014)$. The potential combination of these two methodologies may offer a new model 
for risk assessment capable of harnessing early cellular changes post-exposure to predict potential mixtures effects related to cytotoxicity. From this approach, dose-response chemical mixture cytotoxic effects may be mathematically predicted without measuring the actual mixture. The approach presented in this chapter could be used to test the tens of thousands of chemicals currently used and predict all combinations at multiple doses that have the potential to be synergistic. This would then allow current risk assessment to narrow the experimental efforts to only those doses and chemicals of concern for further in vivo testing

In this proof-of-concept study, we have combined the two methodologies to forecast mixture cell death dose-response using Bliss Independence-predicted phosphorylation values and compared those results to observed phosphorylation response-forecasted cell death and observed cell death values. The inhibitors used as chemical mixtures in this proof of concept study are two mitochondrial electron transport chain (ETC) inhibitors, deguelin $(0.01-100 \mu \mathrm{M})$ and $\mathrm{KCN}(0.01-100 \mu \mathrm{M})$, and two disparate kinase inhibitors, 2-Chloro-3-(N-succinimidyl)-1,4-naphthoquinone, which is a MEK1 inhibitor, commonly referred to as MEK inh II $(0.5-20 \mu \mathrm{M}$ doses $)$ and the substrate-competitive GSK-3 $\beta$ inhibitor, 4-Benzyl-2-methyl-1,2,4-thiadiazolidine-3,5dione, commonly referred to as TDZD-8 (1 - $100 \mu \mathrm{M}$ doses). These mixtures were chosen for their potential interaction and subsequent perturbation of mitochondrial bioenergetics (deguelin and $\mathrm{KCN}$ ), as well as potential intracellular signaling network perturbations (MEK inh II and TDZD-8). The phosphoprotein targets used in this study are kinases involved in cellular survival and/or death pathways. By monitoring the response of these phosphoproteins at critical signaling time-points, the eventual cellular 
fate may be forecasted. Additionally, by using Bliss Independence-predicted phosphoprotein responses for two-way cluster analyses, cell death in response to a potential mixture may be forecasted without experimentally measuring the mixture.

\subsection{Materials and Methods}

\subsubsection{Materials - Deguelin and KCN}

Deguelin (CAS 522-17-8) and KCN (CAS 151-50-8) were obtained from Sigma Aldrich (St. Louis, MO). RPMI-1640 containing phenol red, RPMI-1640 without phenol red, sodium pyruvate, HEPES, L-glutamine, fetal bovine serum, and penicillinstreptomycin were obtained from Invitrogen (Carlsbad, CA). HepG2 cells were obtained from American Type Culture Collection (Manassas, VA). BioPlex beads, lysis buffer, and reagents necessary for determination of relative phosphorylation were obtained from BioRad (Hercules, CA). Deionized water used in this study was prepared with the MilliQ Water System (Millipore, Bedford, MA).

\subsubsection{Materials - MEK inh II and TDZD-8}

The chemicals 4-Benzyl-2-methyl-1,2,4-thiadiazolidine-3,5-dione (TDZD-8, CAS 327036-89-5), Dulbecco's modified Eagle's medium (DMEM), sodium pyruvate, Dglucose, L-glutamate, and sodium bicarbonate were obtained from Sigma Aldrich (St. Louis, MO). 2-Chloro-3-(N-succinimidyl)-1,4-naphthoquinone (MEK inh II, cas 62316352-0) was purchased from CalBiochem (La Jolla, CA, USA). HEPES was purchased 
from Fisher Scientific (USA). Fetal bovine serum, Ethidium homodimer-1 cytotoxicity kit, ATP determination kit (luciferase assay), and penicillin-streptomycin were obtained from Invitrogen (Carlsbad, CA). HepG2 cells were obtained from American Type Culture Collection (Manassas, VA). Multiplex bead-based ELISA assays (beads, reagents, and lysis buffers) were purchased from Bio-Rad (Hercules, CA) and EMD Millipore (Billerica, MA). Deionized water used in this study was prepared with the Milli-Q Water System (Millipore, Bedford, MA).

\subsubsection{Cell culture}

Human hepatocellular carcinoma-derived HepG2 cells were cultured in RPMI1640, supplemented with $1 \mathrm{mM}$ sodium pyruvate, $5 \mathrm{mM}$ HEPES, $2 \mathrm{mM}$ L-Glutamine, $10 \%$ fetal bovine serum, $100 \mathrm{U} / \mathrm{ml}$ penicillin, and $100 \mu \mathrm{g} / \mathrm{ml}$ streptomycin for deguelin and KCN experiments. For MEK inh II and TDZD-8 experiments, HepG2 cells were cultured in DMEM, supplemented with $2 \mathrm{~g} / \mathrm{L}$ D-glucose, $2 \mathrm{mM}$ L-Glutamate, $5 \mathrm{mM}$ HEPES, $24 \mathrm{mM}$ sodium bicarbonate, $1 \mathrm{mM}$ sodium pyruvate, $10 \%$ fetal bovine serum, $100 \mathrm{U} / \mathrm{mL}$ penicillin, and $100 \mathrm{mg} / \mathrm{mL}$ streptomycin. Cells were maintained in a humidified atmosphere at $37^{\circ} \mathrm{C}, 5 \% \mathrm{CO}_{2}$ and passaged at $80 \%$ confluence.

\subsubsection{Dosimetry}

For plasma membrane degradation assays, cells were seeded into clear-bottom, 96-well black-sided plates at a concentration of $4 \times 10^{4}$ cells per well in RPMI (or 
DMEM for MEK inh II/TDZD-8) without phenol red and allowed to grow for $24 \mathrm{~h}$ before dosing. For deguelin and KCN multiplex phosphoprotein assays, cells were seeded in 96-well plates at a concentration of $4 \times 10^{4}$ cells per well in RPMI without phenol red and allowed to grow for $24 \mathrm{~h}$ before dosing. For MEK inh II and TDZD-8 multiplex phosphoprotein assays, cells were seeded in 12-well plates at a concentration of $5 \times 10^{5}$ cells per well in DMEM without phenol red and allowed to grow for $24 \mathrm{~h}$ before dosing. Deguelin, TDZD-8, and MEK inh II dosing solutions were prepared so that resulting well concentrations would be $<1 \%$ DMSO. $\mathrm{KCN}$ is water-soluable, therefore dosing solutions were prepared in water.

\subsubsection{Multiplex phosphoprotein assay - Deguelin and KCN mixtures}

After 400 min of exposure to mixtures of deguelin $(0.01-100 \mu \mathrm{M})$ and $\mathrm{KCN}$ $(0.01-100 \mu \mathrm{M})$, cells were washed with ice cold PBS and lysed according to manufacturer's protocol. Total protein concentration was determined using the DC Protein Assay (BioRad, Hercules, CA) according to the manufacturer's instructions. Protein phosphorylation was determined by using the bead-based BioPlex suspension array system (Bio-Rad, Hercules, CA) and lysates were prepared according to the manufacturer's protocol. Beads and detection antibodies against phosphorylated ERK1/2 (Thr202/Tyr204, Thr185/Tyr187), AKT (Ser473), HSP27 (Ser78), IкBa (Ser32/Ser36), JNK (Thr183/Tyr185), p38MAPK (Thr180/Tyr182), p53 (Ser15), and p90RSK (Thr359/Ser363) were obtained from Bio-Rad (Hercules, CA). Relative phosphorylation 
was calculated by normalizing to control cells, which received dosing vehicle $(<1 \%$ DMSO). All experiments were performed in duplicate.

\subsubsection{Multiplex phosphoprotein assay - MEK inh II and TDZD-8 mixtures}

After 20 min or 40 min of exposure to mixtures of MEK inh II $(0.5,1,2,5,10$, or $20 \mu \mathrm{M})$ and TDZD-8 $(1,5,10,20,50$, or $100 \mu \mathrm{M})$, cells were washed with ice cold PBS and lysed according to manufacturer's protocol. Total protein concentration was determined using the DC Protein Assay (BioRad, Hercules, CA) according to the manufacturer's instructions. Protein phosphorylation was determined by using the beadbased BioPlex suspension array system (Bio-Rad, Hercules, CA) and lysates were prepared according to the manufacturer's protocol. Beads and detection antibodies against phosphorylated AKT (Ser473), ERK1/2 (Thr202/Tyr204, Thr185/Tyr187), HSP27 (Ser78), IкB $\alpha$ (Ser32/Ser36), JNK1/2 (Thr 183/Tyr185), MEK1 (Ser217/Ser221), and p38MAPK (Thr180/Tyr182) were obtained from Bio-Rad (Hercules, CA). Beads and detection antibodies against phosphorylated BAD (Ser 112), CREB1 (Ser133), IRS1 (Ser636/Ser639), and p53(Ser46) were obtained from EMD Millipore. Relative phosphorylation was calculated by normalizing to control cells, which received dosing vehicle (<1\% DMSO). All experiments were performed in duplicate. 


\subsubsection{Plasma membrane degradation assay}

To determine plasma membrane degradation of HepG2 cells exposed to mixtures of deguelin and KCN or MEK inh II and TDZD-8, the ethidium homodimer-1 (EthD-1) kinetic assay was used. Twenty-four hours after seeding HepG2 cells $\left(4 \times 10^{4}\right)$ in a black-sided clear-bottom 96 well plate, culture medium was aspirated and replaced with probe-containing culture medium $(2 \mu \mathrm{M}$ EthD-1). Dead control wells were exposed to $70 \%$ methanol and the plate was incubated for $30 \mathrm{~min}$. Following the 30 minute incubation, cells were challenged mixtures of deguelin $(0.01-100 \mu \mathrm{M})$ and $\mathrm{KCN}(0.01$ $100 \mu \mathrm{M})$ or mixtures of MEK inh II $(0.5,1,2,5,10$, or $20 \mu \mathrm{M})$ and TDZD-8 $(1,5,10$, $20,50$, or $100 \mu \mathrm{M})$ and read immediately after dosing. Fluorescent signal was obtained using an Infinite M1000 microplate reader (Tecan US, Raleigh NC) with excitation wavelength of $530 \mathrm{~nm}$ and emission wavelength of $645 \mathrm{~nm}$, reading from the bottom every $10 \mathrm{~min}$ for $24 \mathrm{~h}$ after dosing. Experiments were performed in duplicate. Relative plasma membrane degradation was determined by normalizing to dead control (received $70 \%$ methanol). Vehicle control wells did not exceed $10 \%$ death.

\subsubsection{Statistical analysis}

Correlation analysis for observed phosphoprotein mixture responses and Bliss independence-predicted phosphoprotein mixture responses were determined using SAS JMP V12Pro (Cary, NC). Two-way hierarchical cluster analyses with Ward's minimum variance were determined by using SAS JMP V12Pro (Cary, NC). Statistical significance for plasma membrane degradation (observed, forecasted, and Bliss-forecasted) were 
determined by using a two-way analysis of variance (ANOVA) with Bonferroni post-test. A difference of $P<0.05$ was considered statistically significant. For plasma membrane degradation and relative phosphorylation data, error bars reflect standard error of the mean (S.E.M.).

\subsection{Results}

3.3.1 Using Bliss independence (response addition) to predict relative phosphorylation during critical signaling events

For this proof-of-concept study, we first determined the relative phosphorylation of HepG2 cells exposed to individual chemicals of interest. Previously, we estimated a critical signaling event for deguelin and $\mathrm{KCN}$ at 400 min post-exposure using $\mathrm{HepG} 2$ cells and relative oxygen consumption observations (Boyd et al., 2013). Since alterations in oxygen consumption (cellular respiration) may suggest early perturbations of cellular bioenergetics and potentially adverse effects, we chose a time point where all dosing conditions experienced the highest degree of change. Previously, we also identified early critical signaling events at $20 \mathrm{~min}$ and $40 \mathrm{~min}$ post-post exposure to MEK inh II and TDZD-8, respectively, in HepG2 cells using our published method for estimating ATP generation (Vrana et al., 2014). At the identified time points post-exposure, a snapshot of the toxicodynamic response to deguelin and $\mathrm{KCN}$ or MEK inh II and TDZD-8 exposure can be determined with a high-throughput approach. Inclusion of valuable proteins that cast a wide net on cell death or survival pathways at this time point is necessary to capture intracellular processes related to adverse or adaptive perturbation responses. 
After exposure to cellular stressors (e.g., xenobiotics, endogenous molecules), many kinase pathways converge upon mitochondria, which can result in mitochondrial membrane permeabilization (MMP) mediated death (Kroemer et al., 2007; Rehm et al., 2009). From this, we chose to narrow the list of potential proteins to those that are only a few kinase steps removed from key survival or death proteins that are known to alter mitochondrial activity. To this end, a multiplex bead-based ELISA assay was designed and used to simultaneously determine different protein phosphorylation responses for deguelin and KCN (Figure 3.1) and MEK inh II and TDZD-8 (Figures 3.2) at time points relevant to their respective critical signaling events. Cells were lysed and measured for phosphoprotein response at the pre-selected critical signaling time points: 400 min postexposure to deguelin and $\mathrm{KCN}$ alone (Figure 3.1) or as a chemical mixture (Appendix

$400 \min$

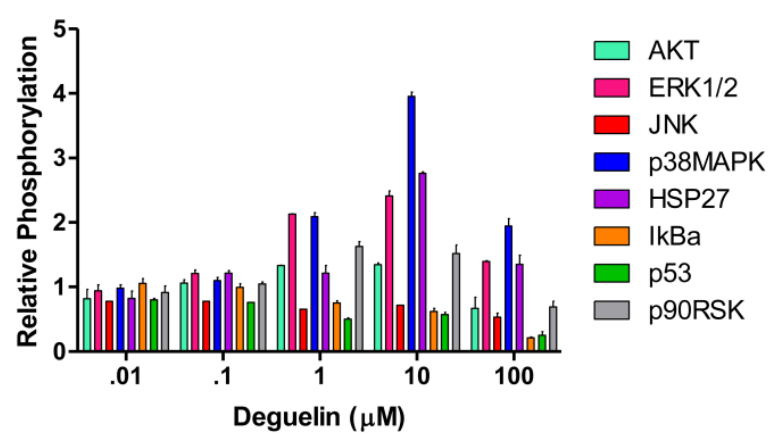

$400 \mathrm{~min}$

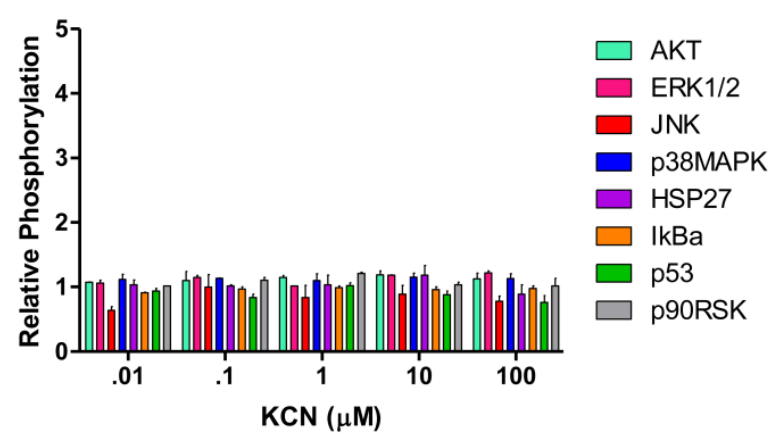

Figure 3.1. Relative phosphorylation responses of HepG2 cells exposed to deguelin or KCN. Relative protein phosphorylation was determined by dosing HepG2 cells with various doses of deguelin $(0.01-100 \mu \mathrm{M})$ or $\mathrm{KCN}(0.01-100 \mu \mathrm{M})$ and lysing the cell membrane at $400 \mathrm{~min}$ post-exposure. Relative phosphorylation was calculated by normalizing to vehicle controls. Error bars reflect S.E.M. Assay was performed in duplicate.

3.1) and 20 min and 40 min post-exposure to MEK inh II and TDZD-8 alone (Figure 2.3) or as a chemical mixture (Appendix 3.2-3.3). Phosphoprotein responses were determined 
relative to control, which received dosing vehicle $(<1 \%$ DMSO for deguelin, MEK inh II, and TDZD-8, or water for KCN).
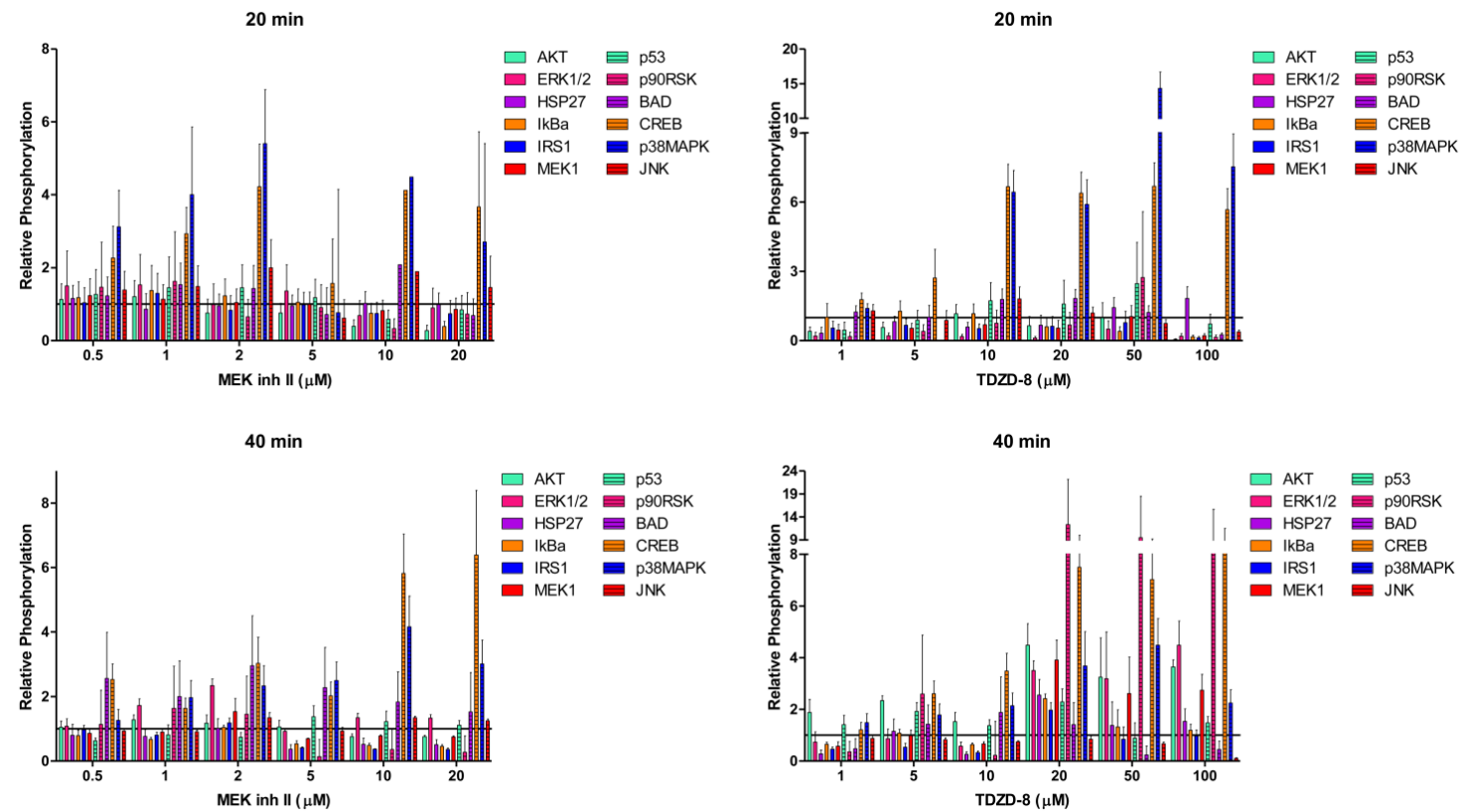

Figure 3.2. Relative phosphorylation responses of HepG2 cells exposed to MEK inh II or TDZD-8. Relative protein phosphorylation was determined by dosing HepG2 cells with various doses of MEK inh II $(0.5,1,2,5,10,20 \mu \mathrm{M}$; left graphs) or TDZD-8 (1, 5, 10, 20, 50, $100 \mu \mathrm{M}$; right graphs) and lysing the cell membrane at $20 \mathrm{~min}$ (top graphs) or $40 \mathrm{~min}$ (bottom graphs) post-exposure. Relative phosphorylation was calculated by normalizing to vehicle controls. Error bars reflect S.E.M. Assay was nerformed in dunlicate. 


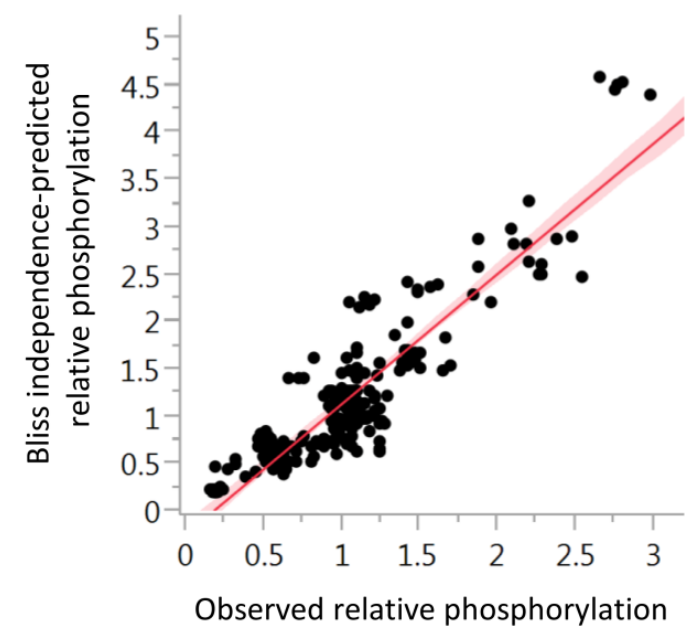

Figure 3.3. Deguelin and KCN mixture correlation. Correlation plot of observed and Bliss Independence-predicted phosphoprotein mixture responses at 400 min post-exposure. Data points represent the mean of the mixture response. For this mixture data set, predicted values were strongly correlated to observed values, $r(200)=0.904, P<.0001$.
For the same mixtures and dosing regimen, we also predicted relative phosphorylation responses at the key time points of interest using Bliss independence (Appendix 3.1-3.3), as previously shown in Boyd, et al., 2013. Experimentally determined phosphoprotein responses of individual chemicals were used to calculate predicted values at the time points of interest. To determine the accuracy of our prediction method, we used correlation analysis for observed and predicted relative

phosphorylation responses (Figures 3.3-3.5). Prediction of relative phosphoprotein responses to deguelin and $\mathrm{KCN}$ mixtures at 400 min post-exposure (Figure 3.3) were

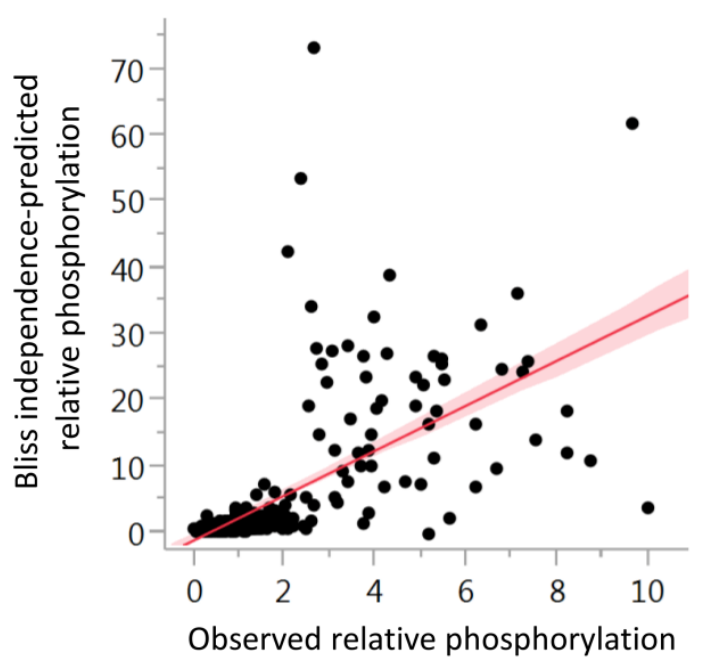

Figure 3.4. MEK inh II and TDZD-8 mixture correlation (20 min). Correlation of observed and Bliss Independence-predicted phosphoprotein mixture responses at $20 \mathrm{~min}$ post-exposure. Data points represent the mean of the mixture response. For this mixture data set, predicted values were moderately correlated to observed values, $r(432)=$ $0.657, P<.0001$.

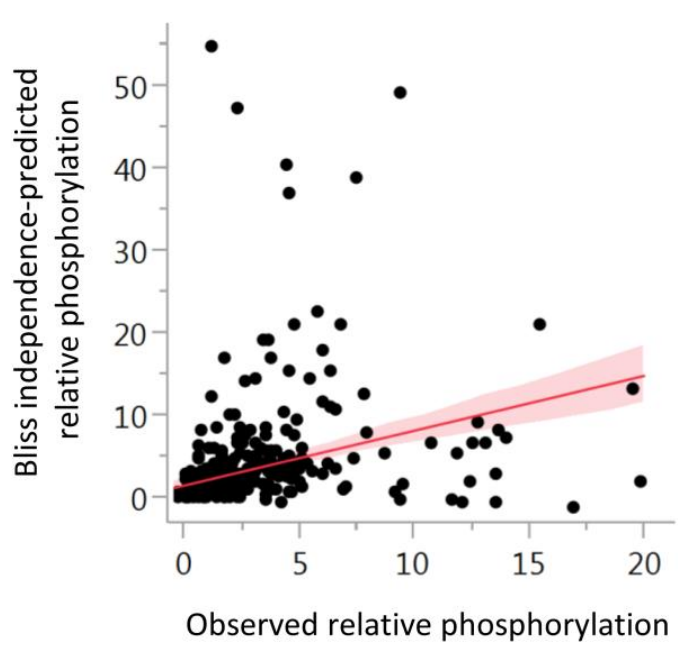

Figure 3.5. MEK inh II and TDZD-8 mixture correlation (40 min). Correlation of observed and Bliss Independence-predicted phosphoprotein mixture responses at $40 \mathrm{~min}$ post-exposure. Data points represent the mean of the mixture response. For this mixture data set, predicted values were weakly correlated to observed values, $r(428)=$ $0.311, P<.0001$. 
strongly correlated to experimentally determined mixture phosphoprotein responses $(r=$ $0.904, \mathrm{~N}=200, P<0.0001)$. Prediction of relative phosphoprotein responses to MEK inh II and TDZD-8 mixtures at 20 min post-exposure (Figure 3.4) were moderately correlated to experimentally determined mixture phosphoprotein responses $(r=0.657, \mathrm{~N}=432, P<$ 0.0001). Prediction of relative phosphoprotein responses to MEK inh II and TDZD-8 mixtures at 40 min post-exposure (Figure 3.5) were weakly correlated to experimentally determined mixture phosphoprotein responses $(r=0.311, \mathrm{~N}=428, P<0.0001)$. The number of data points included in the correlation analysis of MEK inh II and TDZD-8 mixtures at 40 min were 428 instead of 432 due to 4 protein-dose combinations having instrumental error, which in this instance, was from bead aggregation.

\subsubsection{Forecasting mixtures dose-response with hierarchical cluster analysis}

By monitoring or predicting the phosphorylation response of proteins involved in

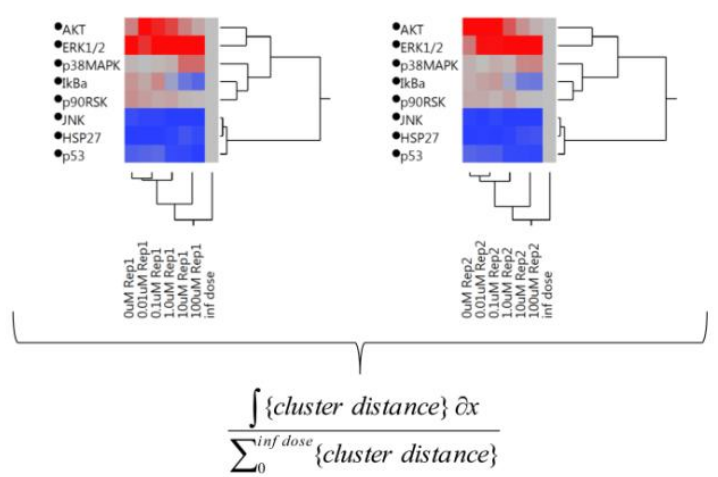

Figure 3.6. Observed phosphoprotein responses for PM degradation forecast. Cluster distances to forecast PM degradation from observed phosphoprotein responses to mixtures of deguelin and $\mathrm{KCN} 400$ min post-exposure were calculated with the unsupervised Ward two-way hierarchical clustering method. PM degradation dose-response curves were calculated using the equation above.

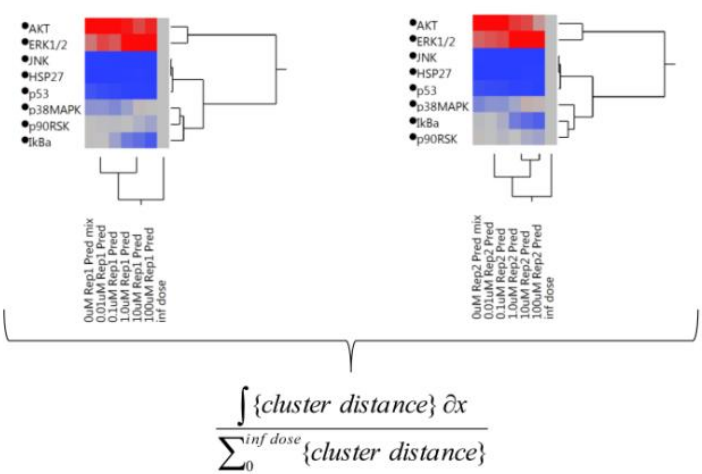

Figure 3.7. Bliss independence-predicted phosphoprotein responses for PM degradation forecast. Cluster distances to forecast PM degradation from predicted phosphoprotein responses to mixtures of deguelin and $\mathrm{KCN} 400$ min post-exposure were calculated with the unsupervised Ward two-way hierarchical clustering method. PM degradation dose-response curves were calculated using the equation above. 
cellular survival and/or death pathways at early time-points, the fate of the cell may be forecasted. In order to accomplish this, we used our previously published method (Vrana et al., 2014), which incorporates two-way cluster analysis with Ward's minimum variance and a transformation of the cluster distances to yield a relative cell death dose-response (for example data analysis, see Figures 3.6-3.7). From this data, relative plasma membrane degradation forecasts can be determined for observed phosphoprotein responses and Bliss independence-predicted responses, and compared to experimentally determined relative plasma membrane degradation (Figure 3.8-3.9).
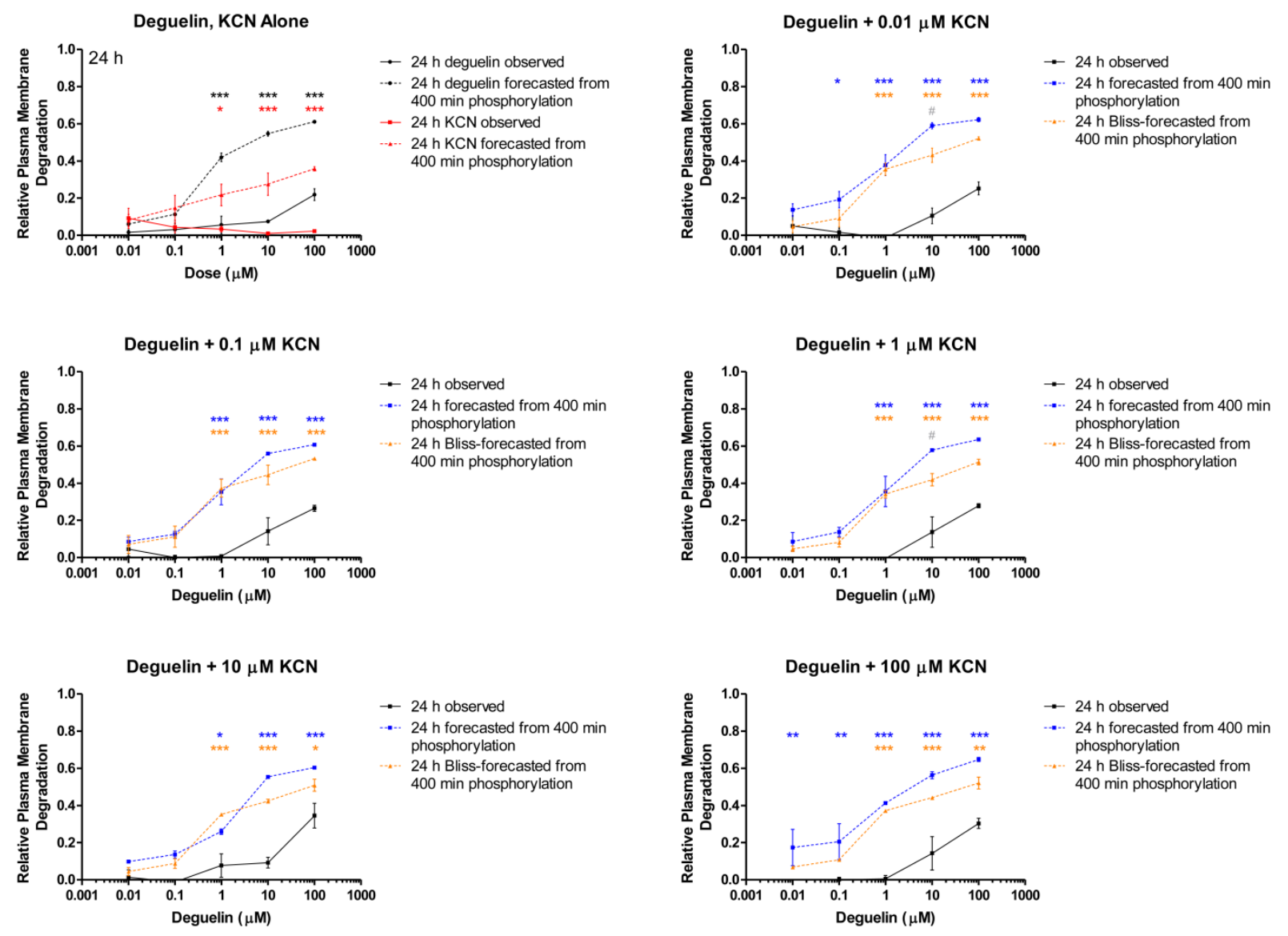

Figure 3.8. Relative PM degradation (predicted and observed) of HepG2 cells at 400 min post-exposure to deguelin and KCN. Relative plasma membrane (PM) degradation dose-response plots depicting the $24 \mathrm{~h}$ observed, $24 \mathrm{~h}$ cluster forecasted PM degradation from observed phosphorylation values at 400 min postexposure, and $24 \mathrm{~h}$ cluster forecasted PM degradation from Bliss Independence-predicted phosphorylation values at $400 \mathrm{~min}$ post-exposure. Forecasted relative PM degradation responses that are significantly different from observed $24 \mathrm{~h}$ PM degradation responses are marked with $*(P<.05)$, ** $(P<.01)$, or $* * *(P<.001)$. Observed relative PM degradation at $400 \mathrm{~min}$ post-exposure had less than $10 \%$ toxicity for all dose combinations measured (data not shown). Forecasted relative PM degradation responses from $400 \mathrm{~min}$ observed phosphorylation responses that were statistically different from Bliss-forecasted relative PM degradation responses from $400 \mathrm{~min}$ predicted phosphoprotein responses were indicated with \# $(P<.05)$. Statistical significance was determined by using two-way ANOVA with Bonferroni post-test. 

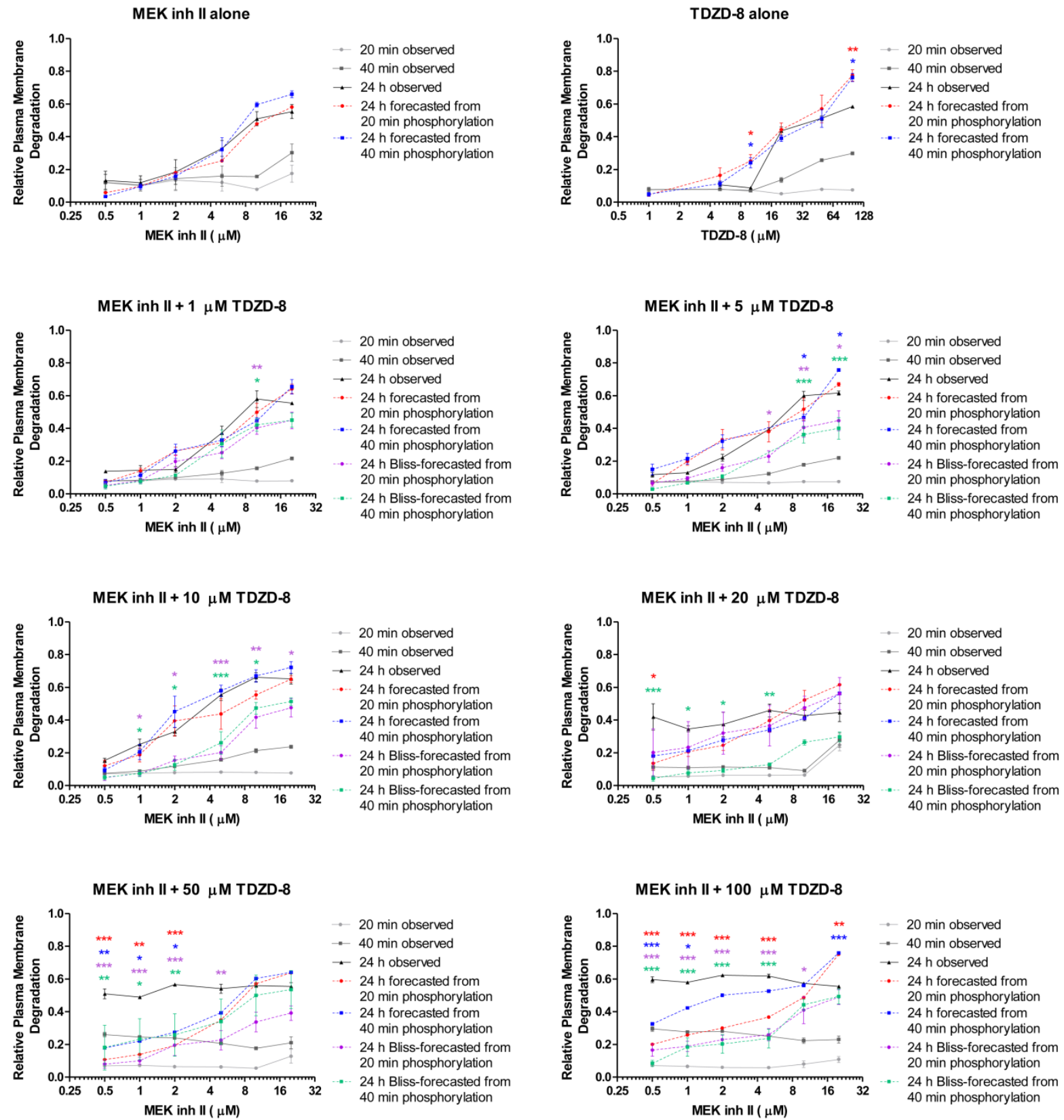

Figure 3.9. Relative PM degradation (predicted and observed) of HepG2 cells at 20 and 40 min post-exposure to MEK inh II and TDZD-8. Relative plasma membrane (PM) degradation dose-response plots depicting the $24 \mathrm{~h}$ observed, $24 \mathrm{~h}$ cluster forecasted PM degradation from observed phosphorylation values at $20 \mathrm{~min}$ (red) and $40 \mathrm{~min}$ (blue) post-exposure, and $24 \mathrm{~h}$ cluster forecasted PM degradation from Bliss Independence-predicted phosphorylation values at $20 \mathrm{~min}$ (purple) and $40 \mathrm{~min}$ (green) post-exposure. Forecasted (from Bliss independence-predicted phosphoprotein responses and observed phosphoprotein responses) relative PM degradation responses that are significantly different from observed $24 \mathrm{~h}$ PM degradation responses are marked with $*(P<.05), * *(P<.01)$, or $* * *(P<.001)$, corresponding to their respective colors. Statistical significance was determined by using two-way ANOVA with Bonferroni post-test. 


\subsection{Discussion}

Humans are continuously exposed to a large number of chemicals, such as food, air, vitamin supplements, and pharmaceuticals, at various doses, and through a variety of exposure routes on a daily basis (Groten et al., 2001). Understanding and ultimately predicting the possible combined effects of a given mixture exposure is necessary for risk assessment toxicology. The current backlog of tens of thousands of chemicals entering the environment for which there is limited or no toxicity information has initiated a paradigm shift in traditional toxicity testing (Wilson and Schwarzman, 2009; Judson et al., 2010; Kavlock et al., 2012; Sipes et al., 2013). This new push towards highthroughput in vitro approach would take advantage of early cellular perturbations postexposure associated with toxicity endpoints in human cell lines and tissues to elucidate mechanistic information regarding the mode(s) of action for a potential xenobiotic or mixture (Attene-Ramos et al., 2013). To this end, we combined our two previous prediction models to develop this proof-of-concept study to monitor early cellular changes relevant to signaling perturbations post-exposure for mixtures model development and potential toxicity predictions.

By combining these two methodologies, we hope to open the door to the possibility of predicting cytotoxicity in response to an exposure of any chemical mixture. For the Bliss-independence predicted phosphorylation responses, the prediction model worked exceptionally well for deguelin and $\mathrm{KCN}$ mixtures as well as MEK inh II and TDZD-8 mixtures after $20 \mathrm{~min}$ of exposure. However, MEK inh II and TDZD-8 predictions were weakly (albeit significantly) correlated to experimentally determined mixture responses after $40 \mathrm{~min}$ of exposure. This model for early PTM toxicodynamic 
responses could greatly benefit from further development since it worked well for certain protein-dose combinations at discrete time points but missed others, especially the MEK inh II and TDZD-8 phosphoprotein responses 40 min post-exposure. As discussed previously in Chapter 1, mixtures prediction models often fail when an observed mixture acts synergistically (Meled et al., 1998; Forget et al., 1999), antagonistically (Posthuma et al., 1997; Van Gestel and Hensbergen, 1997), or experiences dose-dependent subtle interactions (e.g., synergy for low dose mixtures and antagonist for high dose mixtures) (Gennings et al., 2002; Jonker et al., 2004).

In this study, we also utilized our previously developed cytotoxicity forecasting model to potentially predict $24 \mathrm{~h}$ plasma membrane degradation (cell death) with early toxicodynamic perturbations. The deguelin and $\mathrm{KCN}$ mixtures (both observed phosphorylation response forecasted and Bliss-predicted phosphorylation responseforecasted) were only 30 and $40 \%$ accurate for prediction of cytotoxicity, respectively. However, most low dose deguelin mixtures were accurately predicted $(0.01$ and $0.1 \mu \mathrm{M})$. It is important to note that both models for deguelin and $\mathrm{KCN}$ mixtures were not significantly different from each other (only 2 of 25 dose combinations were significantly different, $P<.05)$, suggesting that the Bliss Independence-predicted phosphoprotein responses could be used in lieu of actual phosphoprotein mixture responses to forecast cytotoxicity. For MEK inh II and TDZD-8 mixtures forecasts (20 min), both observed phosphorylation response forecasted and Bliss-predicted phosphorylation responseforecasted were 75 and $50 \%$ accurate for prediction of cytotoxicity, respectively. Also, for MEK inh II and TDZD-8 mixtures forecasts (40 min), both observed phosphorylation response forecasted and Bliss-predicted phosphorylation response-forecasted were 77 and 
$49 \%$ accurate for prediction of cytotoxicity, respectively. The early cellular changes measured (i.e., phosphorylation responses of kinases related to cell death and/or survival pathways) at 20 min post exposure were better for predicting cytotoxicity than at 40 min post-exposure to mixtures of MEK inh II and TDZD-8. This shows that appropriate selection of early time points, as well as judiciously selected phosphoproteins may improve prediction models. Most imporantly, the forecasted $24 \mathrm{~h}$ PM degradation using Bliss-independence predicted phosphoprotein responses accurately forecasted cytotoxicity (77\% and $49 \%$ for $20 \mathrm{~min}$ and $40 \mathrm{~min}$ data, respectively). This suggests that cytotoxicity dose-response of mixtures can be estimated without measuring the actual mixture. The mixture predictions for MEK inh II and TDZD-8 were much more accurate than the deguelin and $\mathrm{KCN}$ mixture predictions $(49-77 \%$ vs $30-40 \%)$. This could be due to the inclusion of 12 phosphoproteins for MEK inh II and TDZD-8 mixtures as opposed to the 8 phosphoproteins for deguelin and KCN. Additionally, MEK inh II and TDZD-8 mixture critical signaling time points were selected from estimated intracellular ATP, whereas deguelin and $\mathrm{KCN}$ mixture critical signaling time points were selected from oxygen consumption alone.

Possible improvements to this approach could be in the type and number of PTMs to measure for early cellular perturbations post-exposure. Previously, our forecast model using individual compounds was very successful by using 19 phosphoprotein responses. However, in this study, only 8 and 12 phosphoproteins were included for mixture predictions. Future optimization of the number and identification of proteins necessary for accurate predictions will dramatically improve our model for cytotoxicity forecasting. 
The initial success of this proof-of-concept approach suggests that highthroughput in vitro assays incorporating a wide range of doses (low, moderate, and high) and early cellular changes to predict individual chemical and mixtures toxicity is exceedingly advantageous. We believe that this will greatly impact xenobiotic highthroughput mixtures risk assessment in vitro, as well as pharmaceutical adverse drug reaction analyses.

\subsection{References}

Andersen, M.E., Krewski, D., 2009. Toxicity testing in the 21 st century: bringing the vision to life. Toxicol Sci 107, 324-330.

Attene-Ramos, M.S., Miller, N., Huang, R., Michael, S., Itkin, M., Kavlock, R.J., Austin, C.P., Shinn, P., Simeonov, A., Tice, R.R., Xia, M., 2013. The Tox 21 robotic platform for the assessment of environmental chemicals--from vision to reality. Drug Discov Today 18, 716-723.

Bhattacharya, S., Zhang, Q., Carmichael, P.L., Boekelheide, K., Andersen, M.E., 2011. Toxicity testing in the 21 century: defining new risk assessment approaches based on perturbation of intracellular toxicity pathways. PLoS One 6, e20887.

Boyd, J., Vrana, J.A., Williams, H.N., 2013. In vitro approach to predict posttranslational phosphorylation response to mixtures. Toxicology 313, 113-121.

Bushnell, P.J., Kavlock, R.J., Crofton, K.M., Weiss, B., Rice, D.C., 2010. Behavioral toxicology in the 21st century: challenges and opportunities for behavioral scientists. Summary of a symposium presented at the annual meeting of the neurobehavioral teratology society, June, 2009. Neurotoxicol Teratol 32, 313-328.

Cedergreen, N., Christensen, A.M., Kamper, A., Kudsk, P., Mathiassen, S.K., Streibig, J.C., Sorensen, H., 2008. A review of independent action compared to concentration addition as reference models for mixtures of compounds with different molecular target sites. Environ Toxicol Chem 27, 1621-1632.

Dix, D.J., Houck, K.A., Martin, M.T., Richard, A.M., Setzer, R.W., Kavlock, R.J., 2007. The ToxCast program for prioritizing toxicity testing of environmental chemicals. Toxicol Sci 95, 5-12. 
Forget, J., Pavillon, J.-F., Beliaeff, B., Bocquene, G., 1999. Joint action of pollutant combinations (pesticides and metals) on survival (LC50 values) and acetylcholinesterase activity of Tigriopus brevicornis (Copepoda, Harpacticoida). Environ Toxicol Chem 18, 912-918.

Gennings, C., Carter, W., Campain, J., Bae, D., Yang, R., 2002. Statistical analysis of interactive cytotoxicity in human epidermal ke. J Agric Biol Environ Stat 7, 5873.

Groten, J.P., Feron, V.J., Suhnel, J., 2001. Toxicology of simple and complex mixtures. Trends Pharmacol Sci 22, 316-322.

Hartung, T., 2009. Toxicology for the twenty-first century. Nature 460, 208-212.

Jonker, M.J., Piskiewicz, A.M., Ivorra i Castella, N., Kammenga, J.E., 2004. Toxicity of binary mixtures of cadmium-copper and carbendazim-copper to the nematode Caenorhabditis elegans. Environ Toxicol Chem 23, 1529-1537.

Judson, R.S., Houck, K.A., Kavlock, R.J., Knudsen, T.B., Martin, M.T., Mortensen, H.M., Reif, D.M., Rotroff, D.M., Shah, I., Richard, A.M., Dix, D.J., 2010. In vitro screening of environmental chemicals for targeted testing prioritization: the ToxCast project. Environ Health Perspect 118, 485-492.

Kavlock, R., Chandler, K., Houck, K., Hunter, S., Judson, R., Kleinstreuer, N., Knudsen, T., Martin, M., Padilla, S., Reif, D., Richard, A., Rotroff, D., Sipes, N., Dix, D., 2012. Update on EPA's ToxCast program: providing high throughput decision support tools for chemical risk management. Chem Res Toxicol 25, 1287-1302.

Krewski, D., Acosta, D., Jr., Andersen, M., Anderson, H., Bailar, J.C., 3rd, Boekelheide, K., Brent, R., Charnley, G., Cheung, V.G., Green, S., Jr., Kelsey, K.T., Kerkvliet, N.I., Li, A.A., McCray, L., Meyer, O., Patterson, R.D., Pennie, W., Scala, R.A., Solomon, G.M., Stephens, M., Yager, J., Zeise, L., 2010. Toxicity testing in the 21st century: a vision and a strategy. J Toxicol Environ Health B Crit Rev 13, 51138.

Kroemer, G., Galluzzi, L., Brenner, C., 2007. Mitochondrial membrane permeabilization in cell death. Physiol Rev 87, 99-163.

Meled, M., Thrasyvoulou, A., Belzunces, L.P., 1998. Seasonal variations in susceptibility of Apis mellifera to the synergistic action of prochloraz and deltamethrin. Environ Toxicol Chem 17, 2517-2520.

NRC, 2007. Toxicity Testing in the 21st Century: A Vision and a Strategy. National Academies Press, Washington, D.C. 
Posthuma, L., Baerselman, R., Van Veen, R.P., Dirven-Van Breemen, E.M., 1997. Single and joint toxic effects of copper and zinc on reproduction of Enchytraeus crypticus in relation to sorption of metals in soils. Ecotoxicol Environ Saf 38, 108-121.

Rehm, M., Huber, H.J., Hellwig, C.T., Anguissola, S., Dussmann, H., Prehn, J.H., 2009. Dynamics of outer mitochondrial membrane permeabilization during apoptosis. Cell Death Differ 16, 613-623.

Sipes, N.S., Martin, M.T., Kothiya, P., Reif, D.M., Judson, R.S., Richard, A.M., Houck, K.A., Dix, D.J., Kavlock, R.J., Knudsen, T.B., 2013. Profiling 976 ToxCast chemicals across 331 enzymatic and receptor signaling assays. Chem Res Toxicol 26, 878-895.

Van Gestel, C.A.M., Hensbergen, P.J., 1997. Interaction of Cd and Zn toxicity for Folsomia candida Willem (Collembola:Isotomidae) in relation to bioavailability in soil. Environ Toxicol Chem 16, 1177-1186.

Vrana, J.A., Currie, H.N., Han, A.A., Boyd, J., 2014. Forecasting cell death doseresponse from early signal transduction responses in vitro. Toxicol Sci 140, 338351.

Wilson, M.P., Schwarzman, M.R., 2009. Toward a new U.S. chemicals policy: rebuilding the foundation to advance new science, green chemistry, and environmental health. Environ Health Perspect 117, 1202-1209. 
Appendix 3.1 - Observed relative phosphorylation of deguelin and $\mathrm{KCN}$ mixtures (400 min post-exposure)

$$
\text { Deguelin + } 0.01 \mu \mathrm{M} \mathrm{KCN}(400 \mathrm{~min})
$$

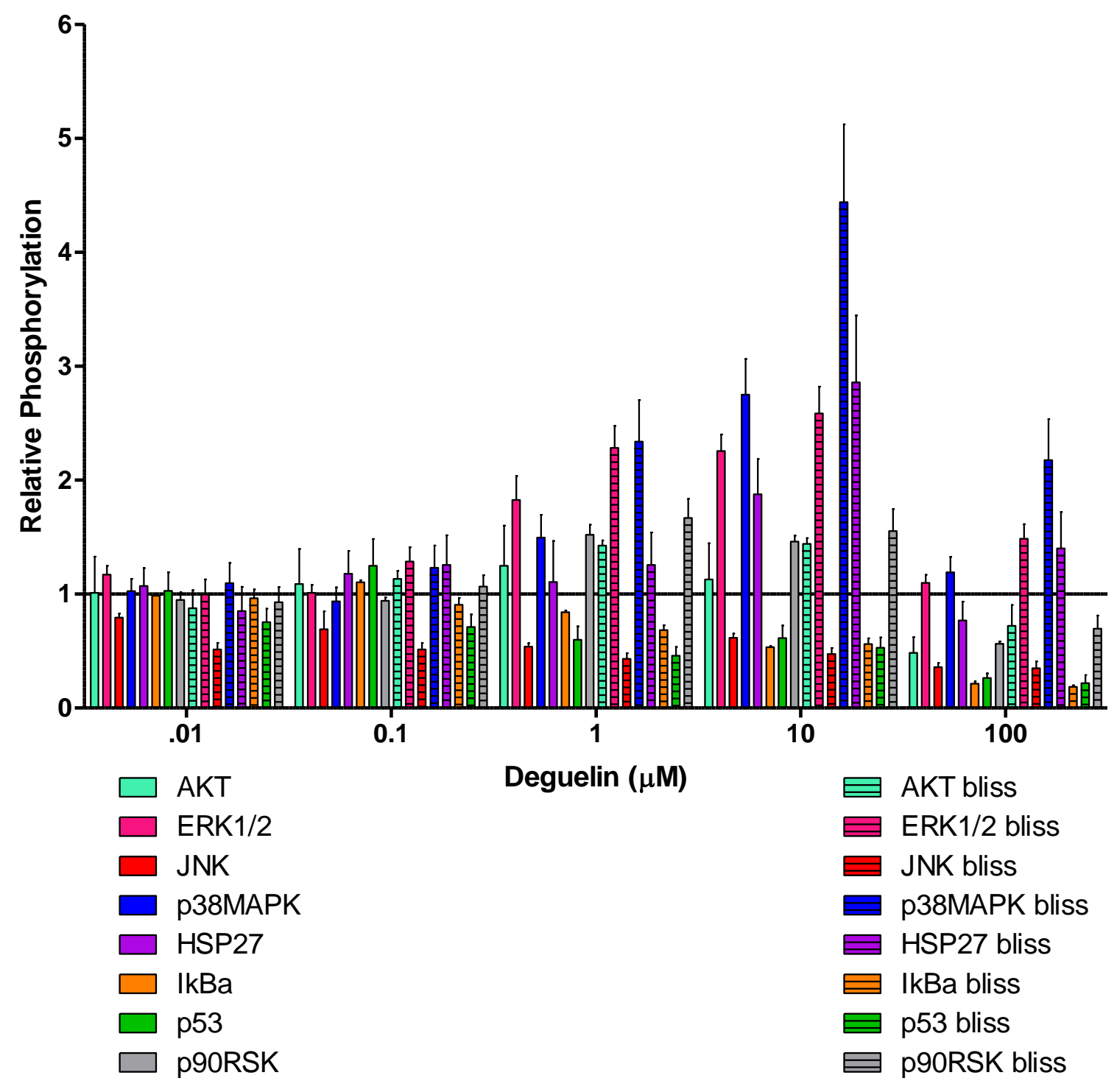




\section{Deguelin + 0.1 $\mu$ M KCN (400 min)}

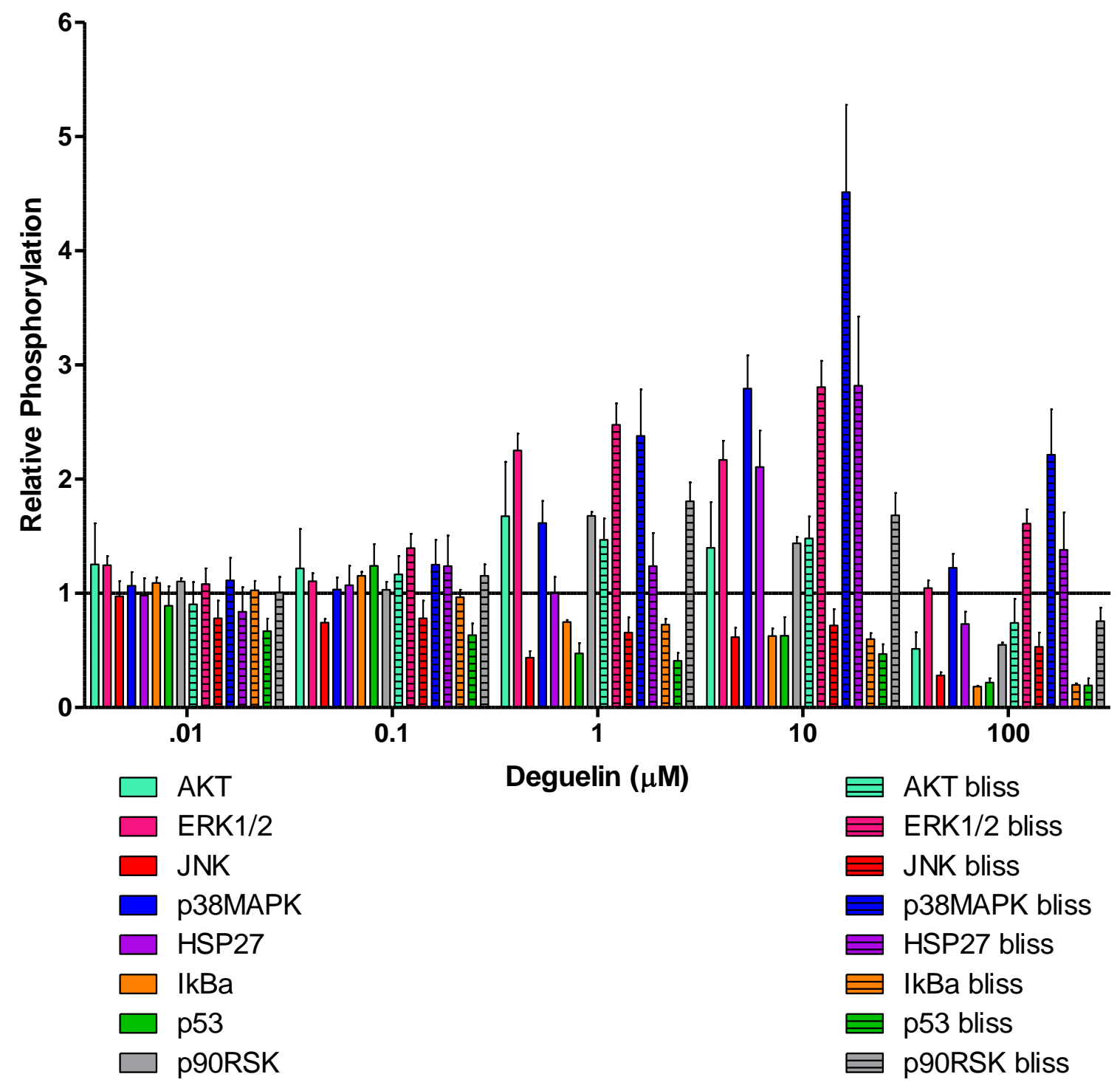


Deguelin + $1 \mu$ M KCN (400 min)

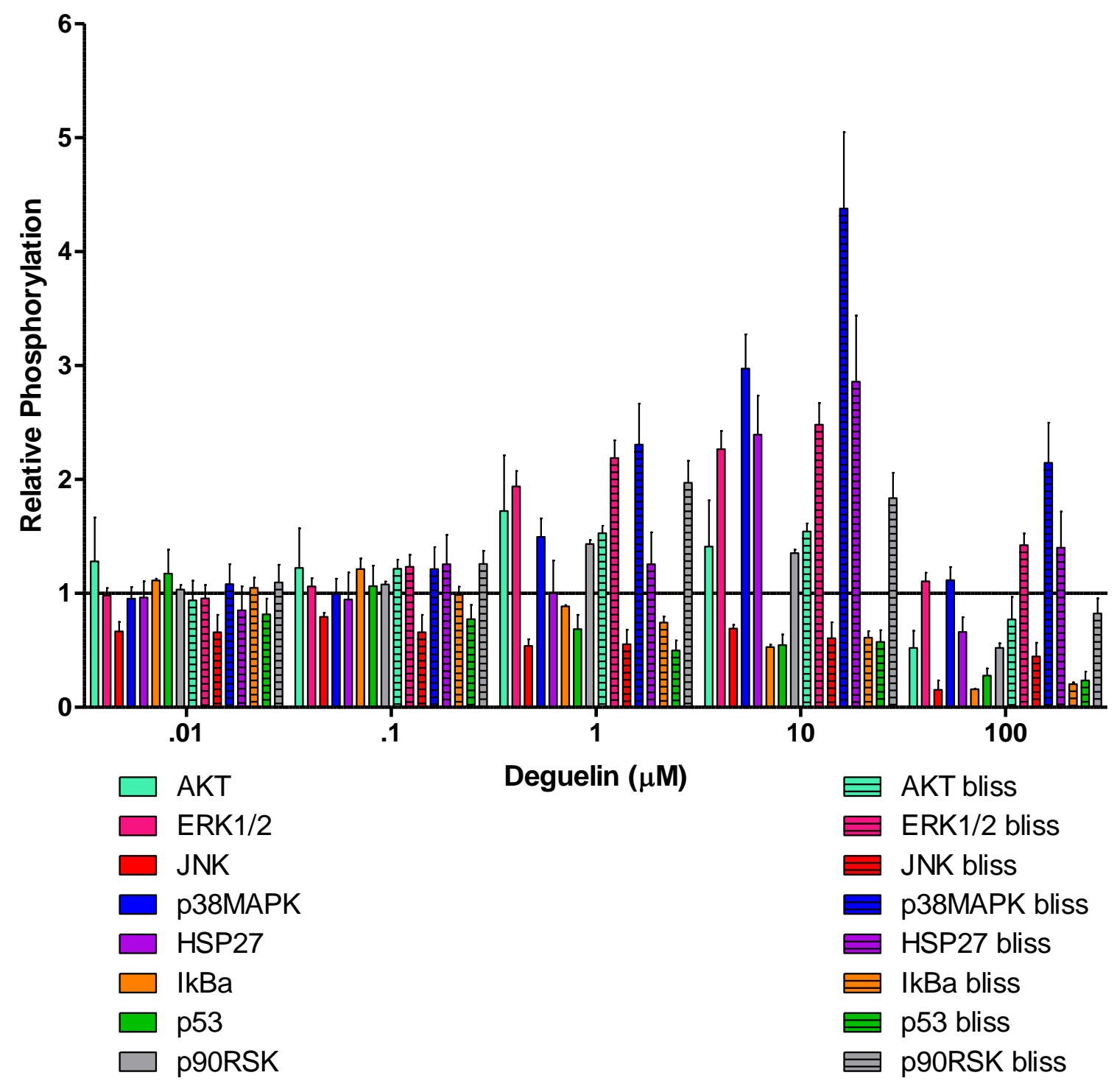


Deguelin + $10 \mu \mathrm{M} \mathrm{KCN} \mathrm{(400} \mathrm{min)}$

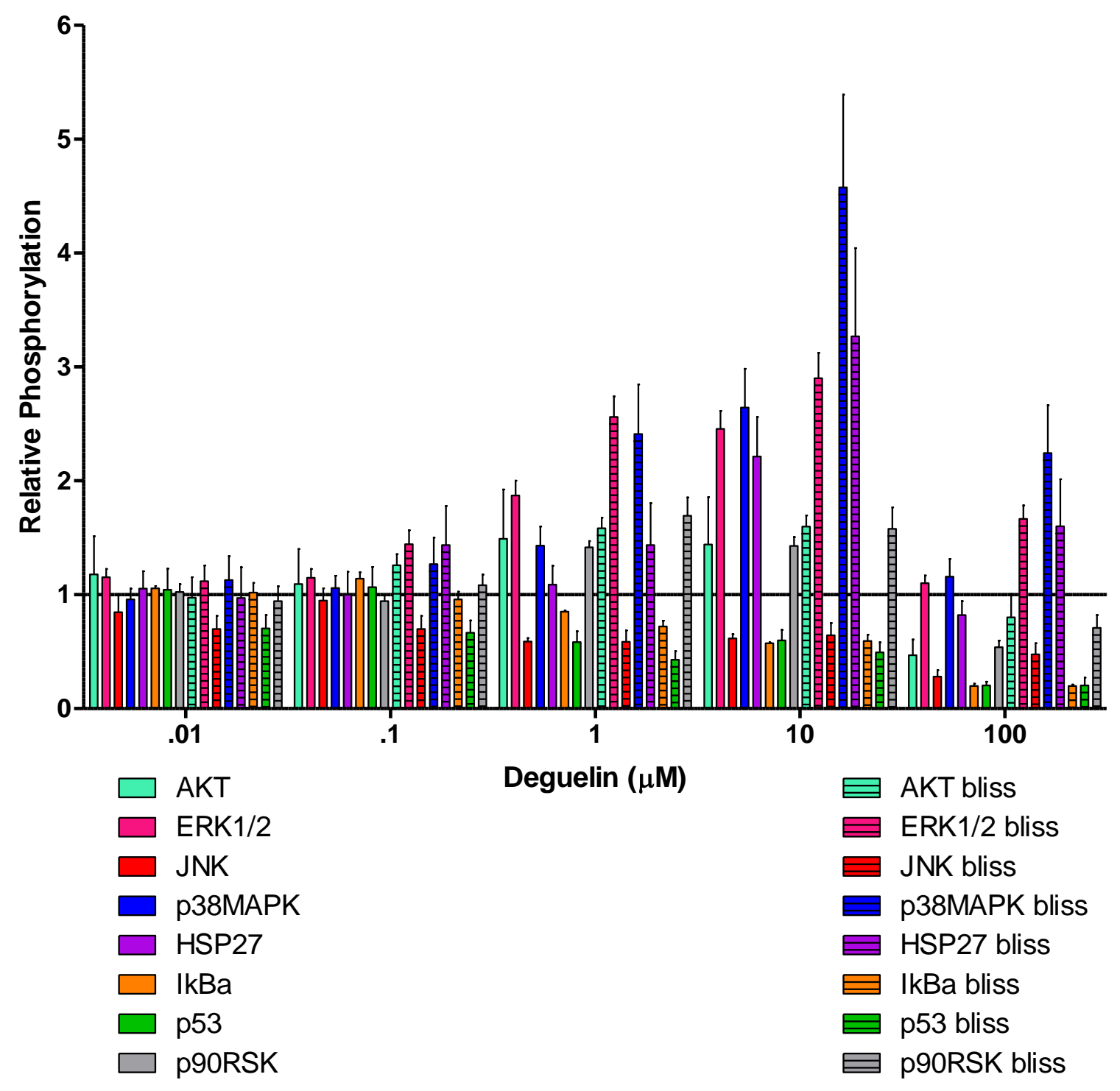




\section{Deguelin $+100 \mu \mathrm{M} \mathrm{KCN} \mathrm{(400} \mathrm{min)}$}

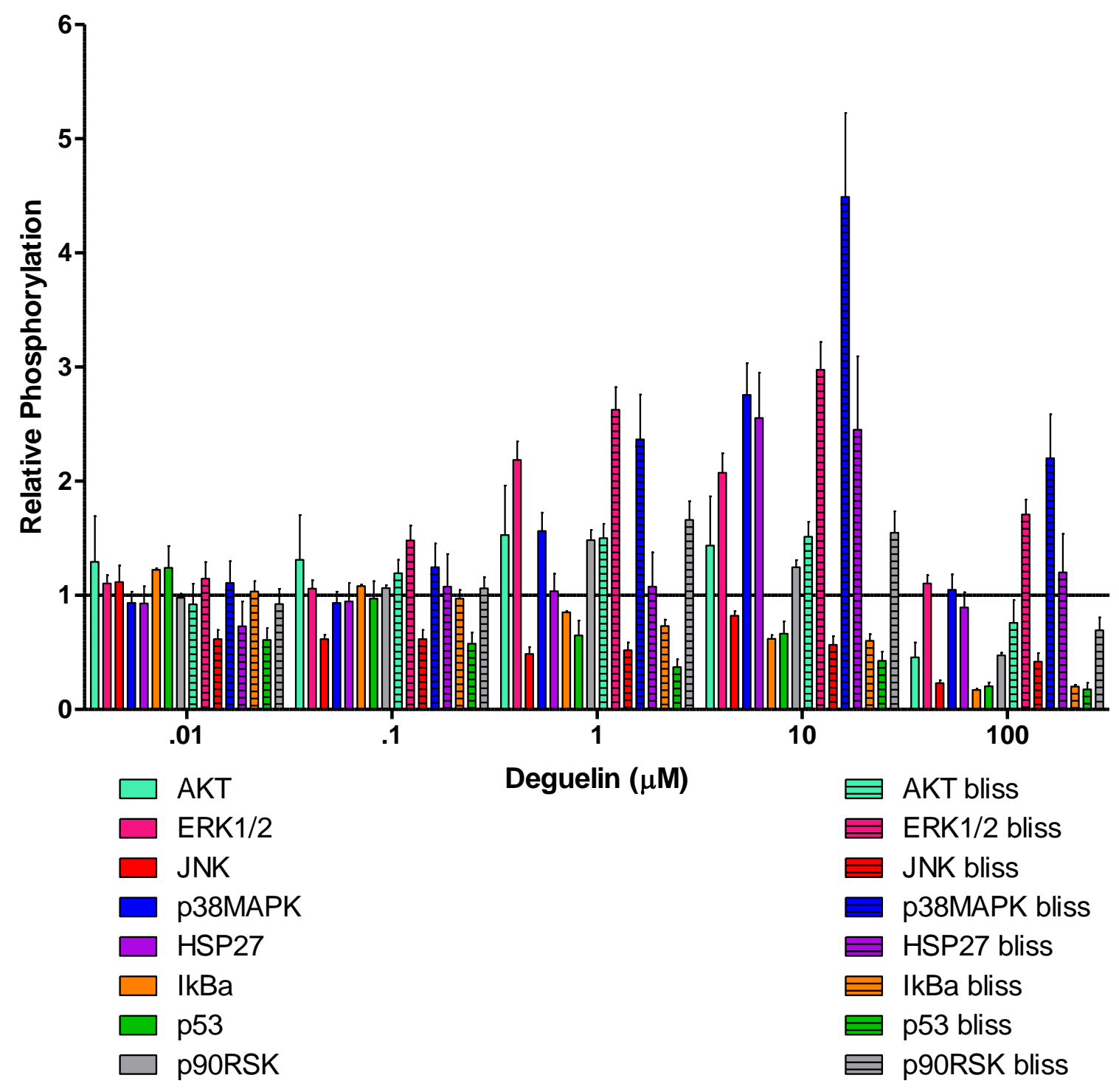


Appendix 3.2 - Observed relative phosphorylation of MEK inh II and TDZD-8 mixtures (20 min post-exposure)

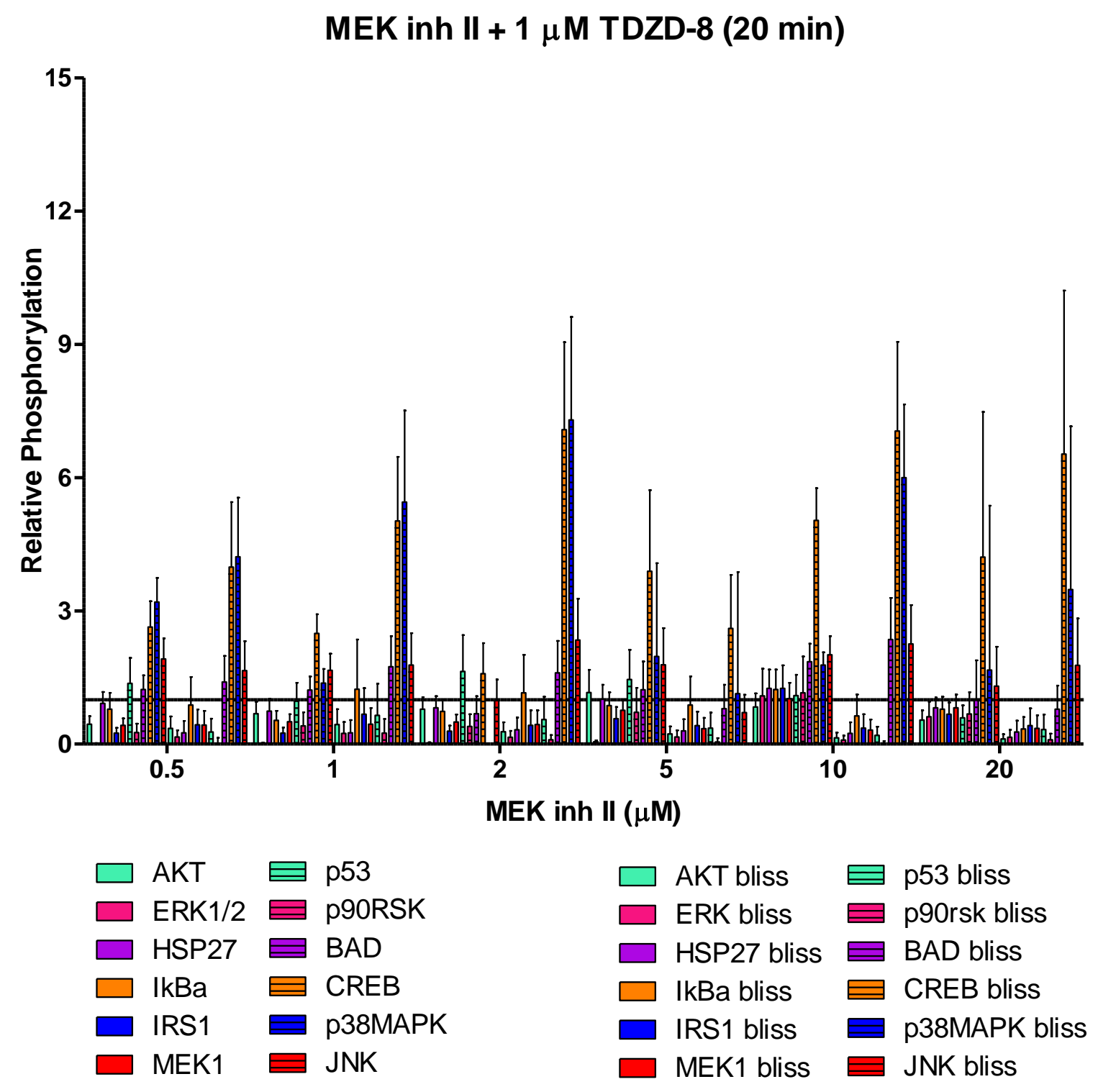




\section{MEK inh II + $5 \mu$ M TDZD-8 (20 min)}

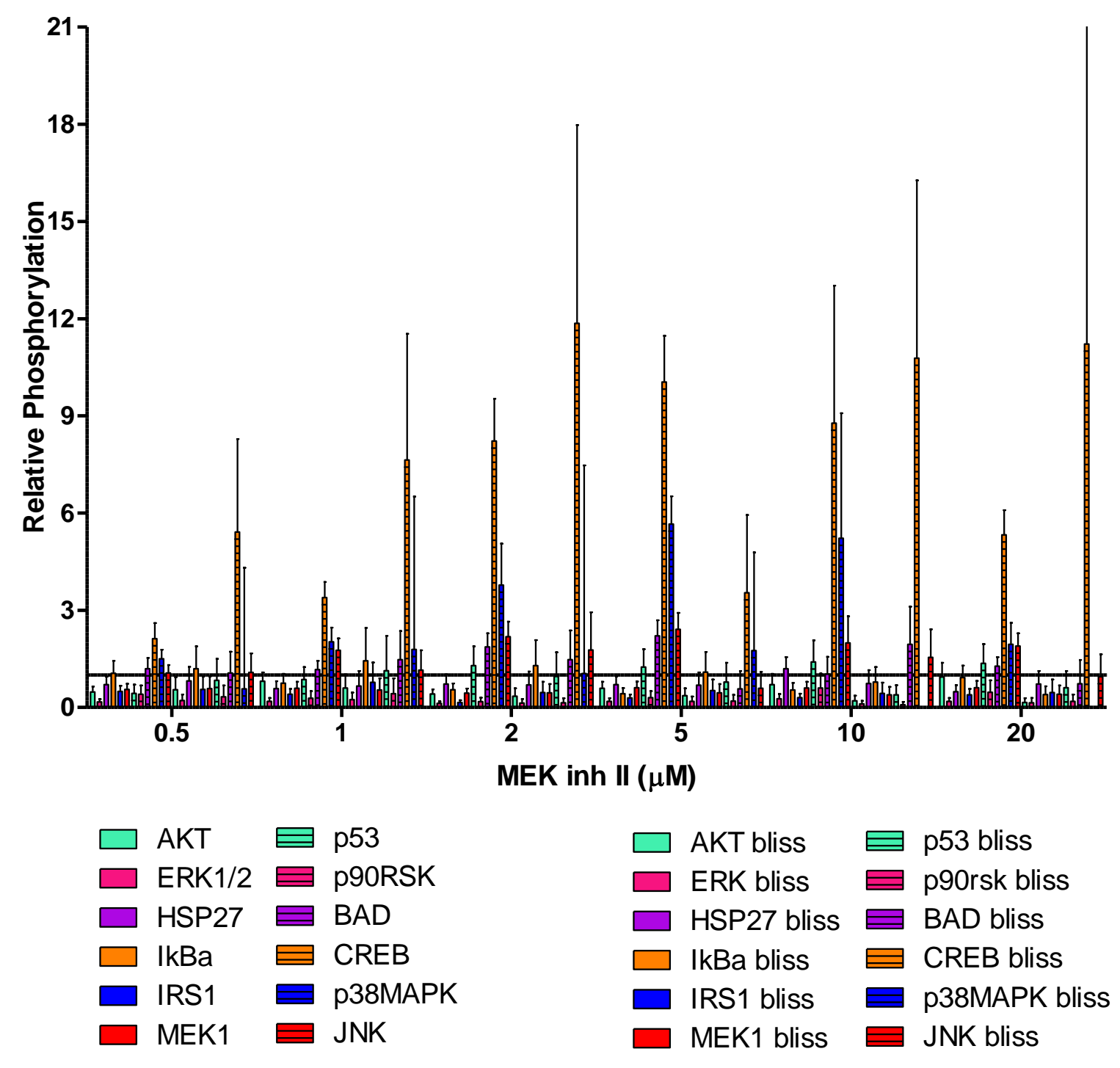




\section{MEK inh II + $10 \mu M$ TDZD-8 (20 min)}
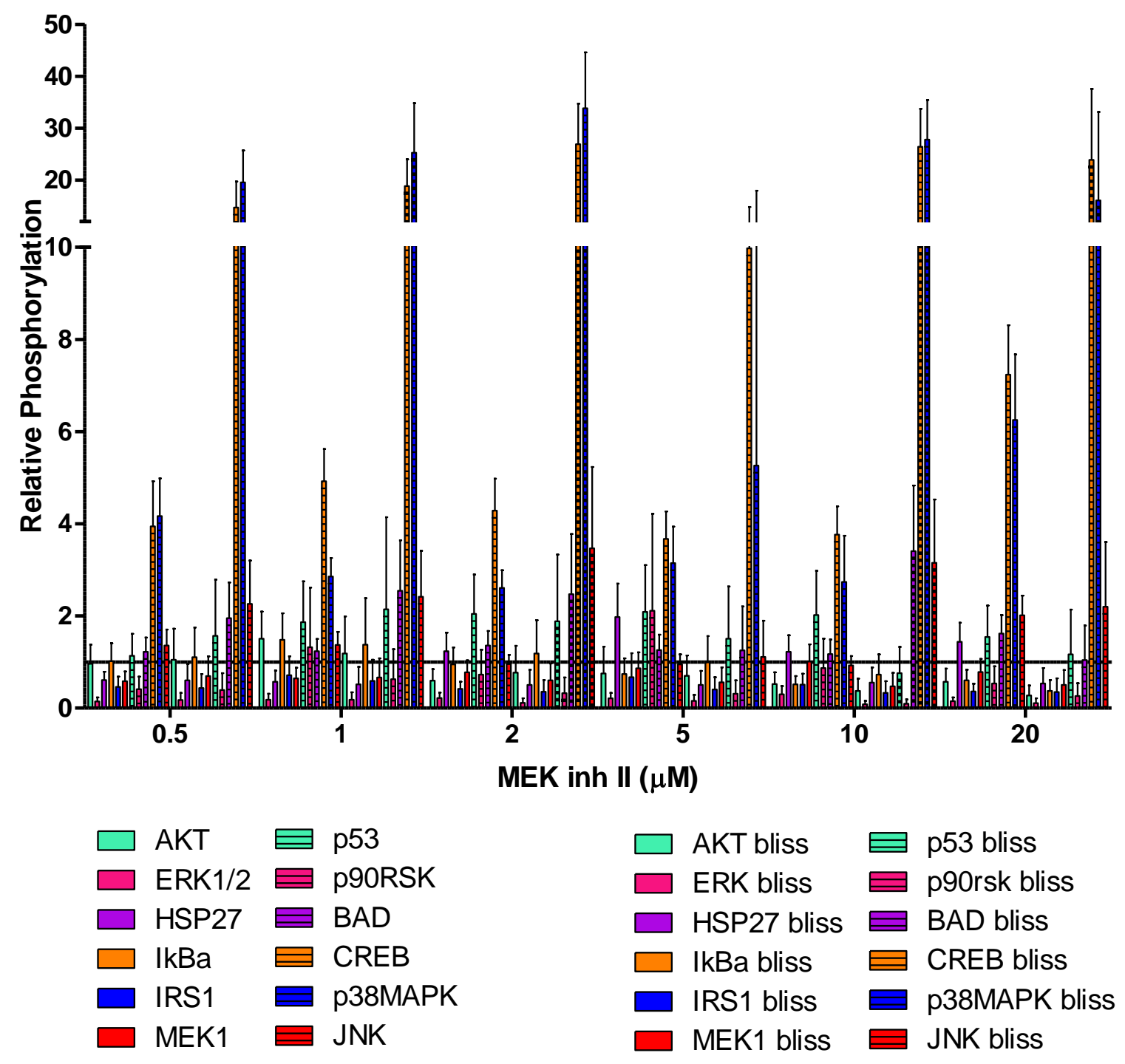

\section{MEK inh II $(\mu \mathrm{M})$}

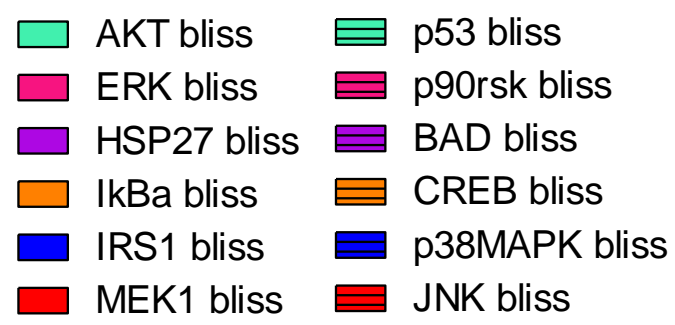




\section{MEK inh II + $20 \mu M$ TDZD-8 (20 min)}

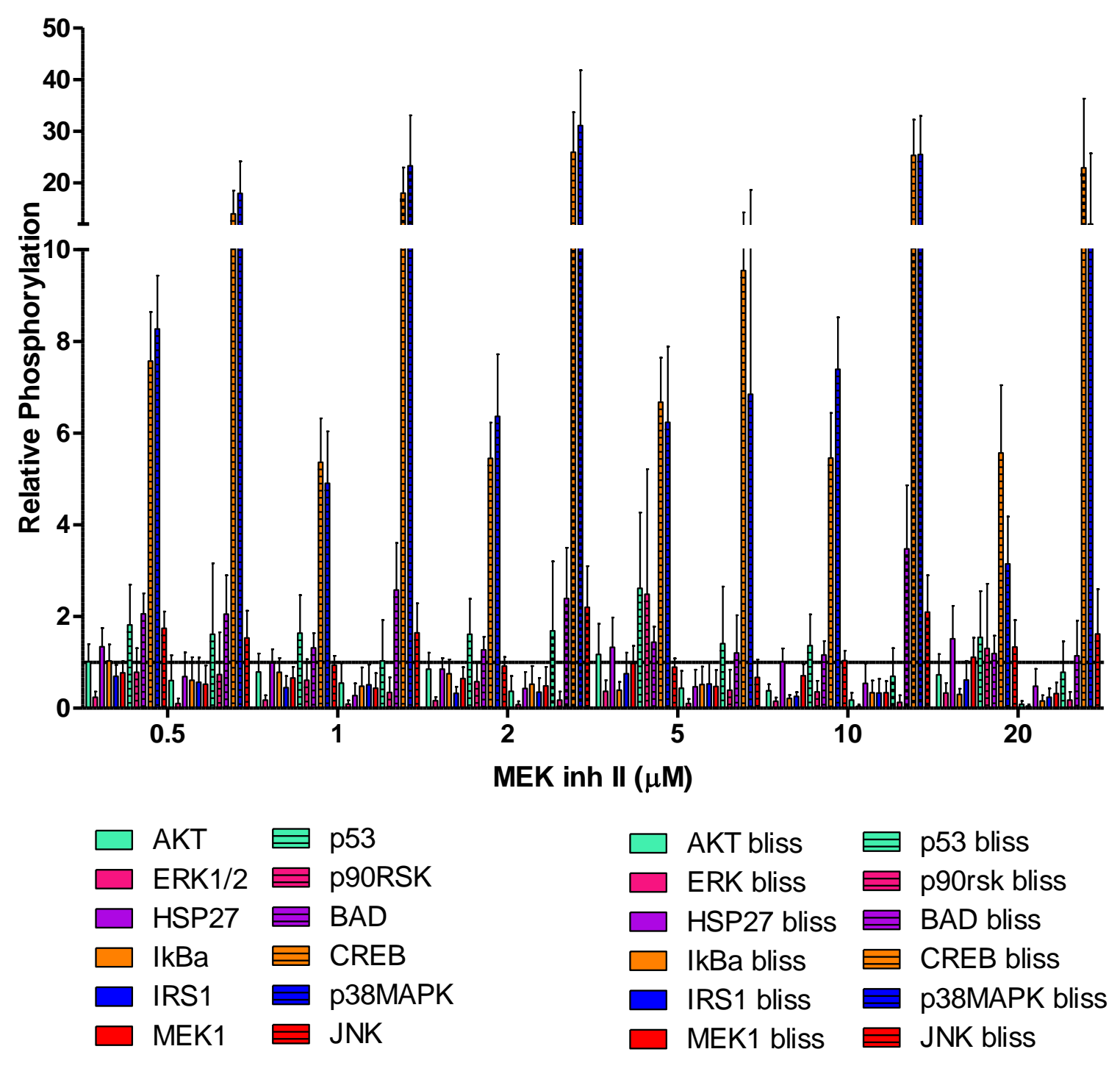




\section{MEK inh II + $50 \mu$ M TDZD-8 (20 min)}
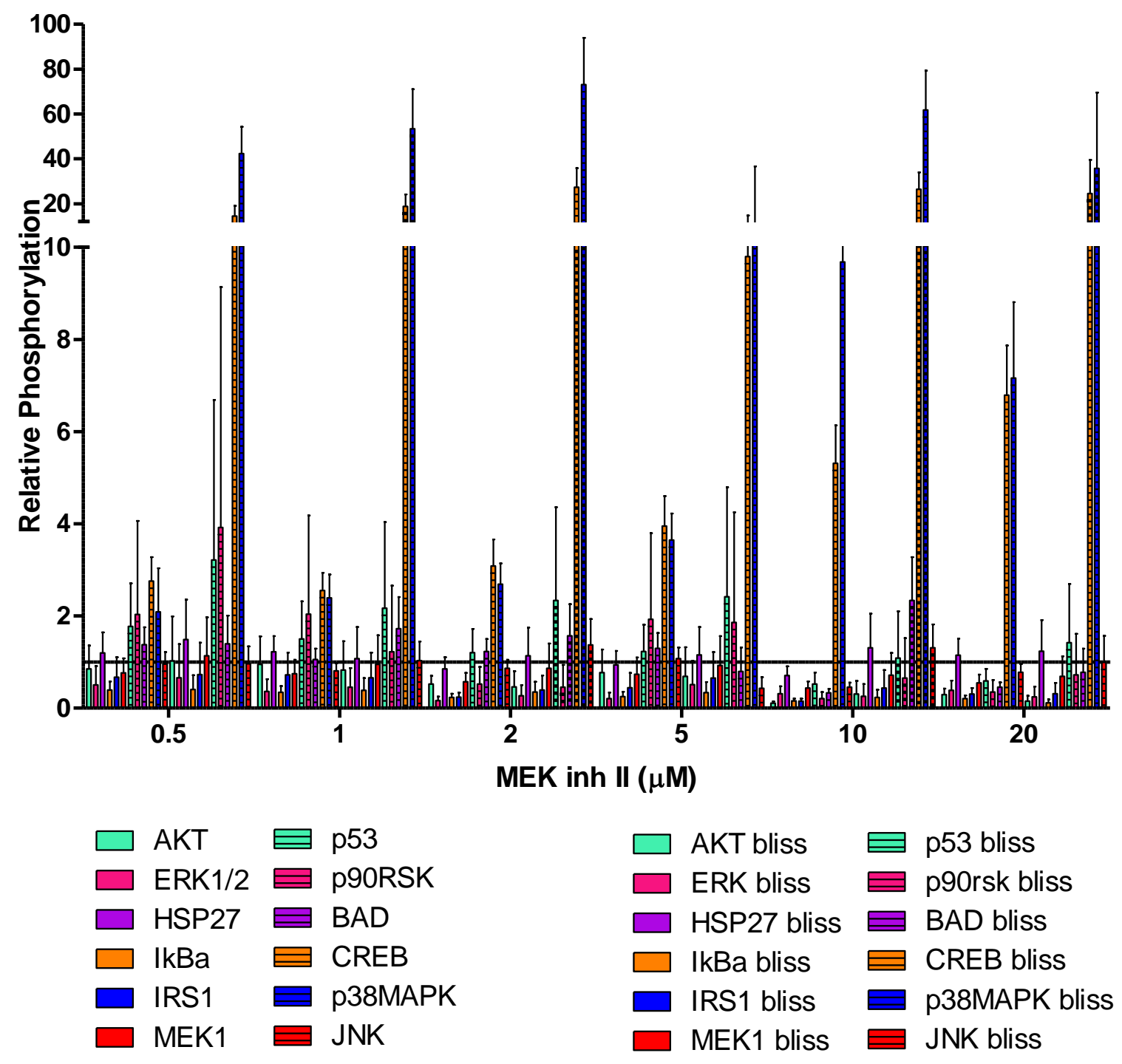

MEK inh II $(\mu \mathrm{M})$

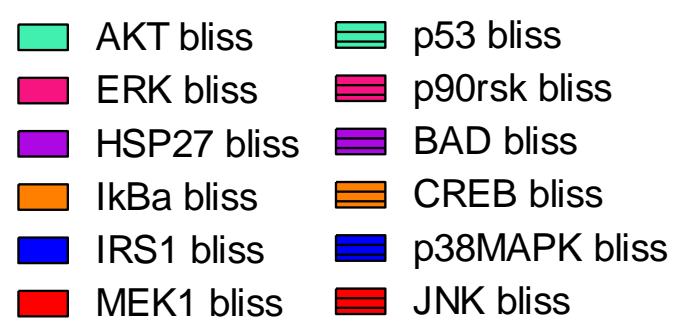




\section{MEK inh II + $100 \mu$ M TDZD-8 (20 min)}

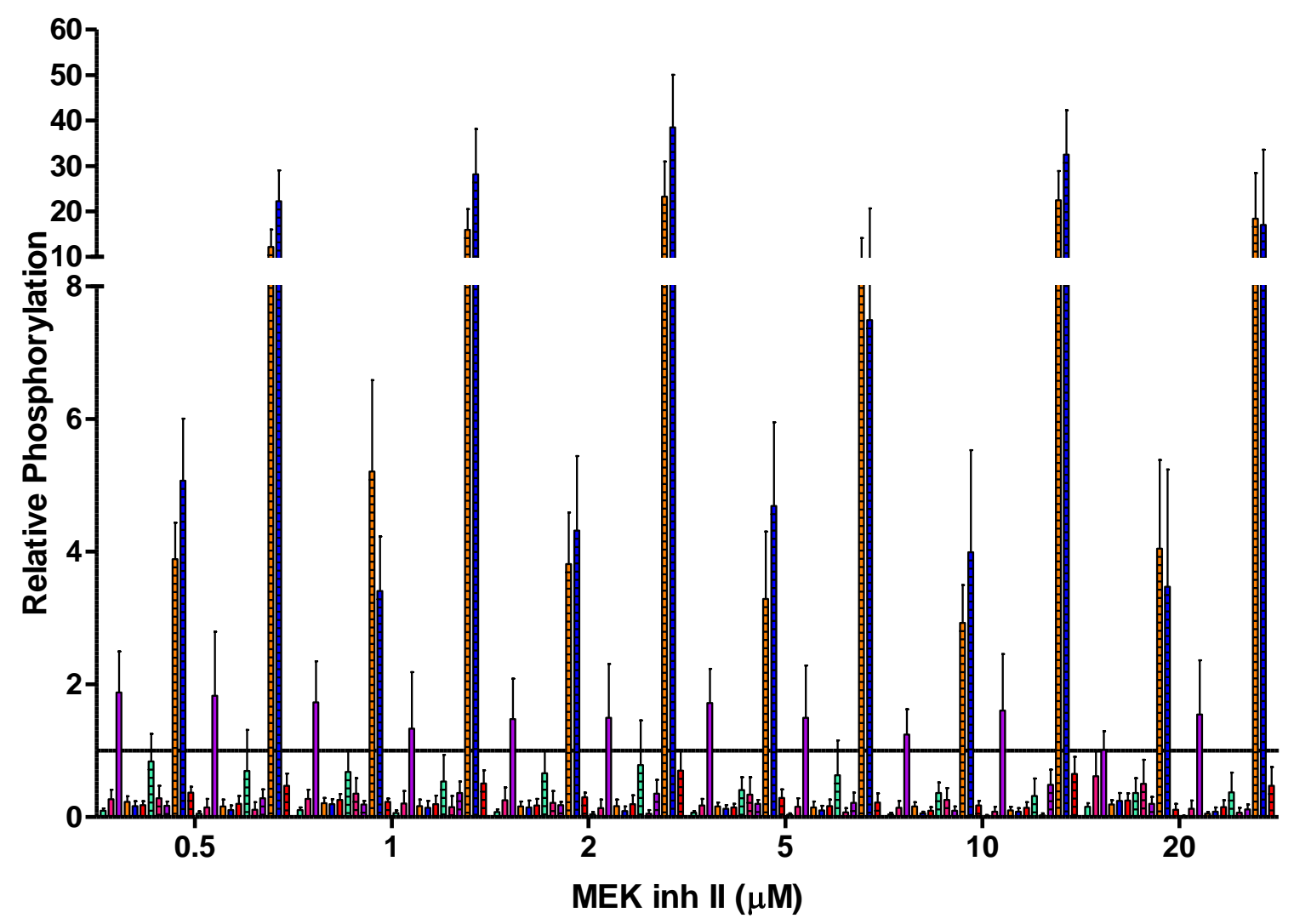

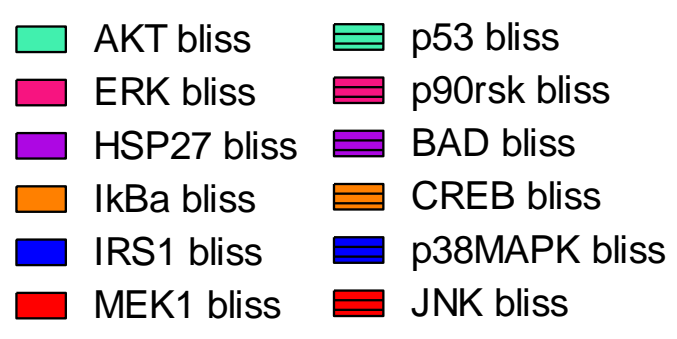


Appendix 3.3 - Observed relative phosphorylation of MEK inh II and TDZD-8 mixtures (40 min post-exposure)

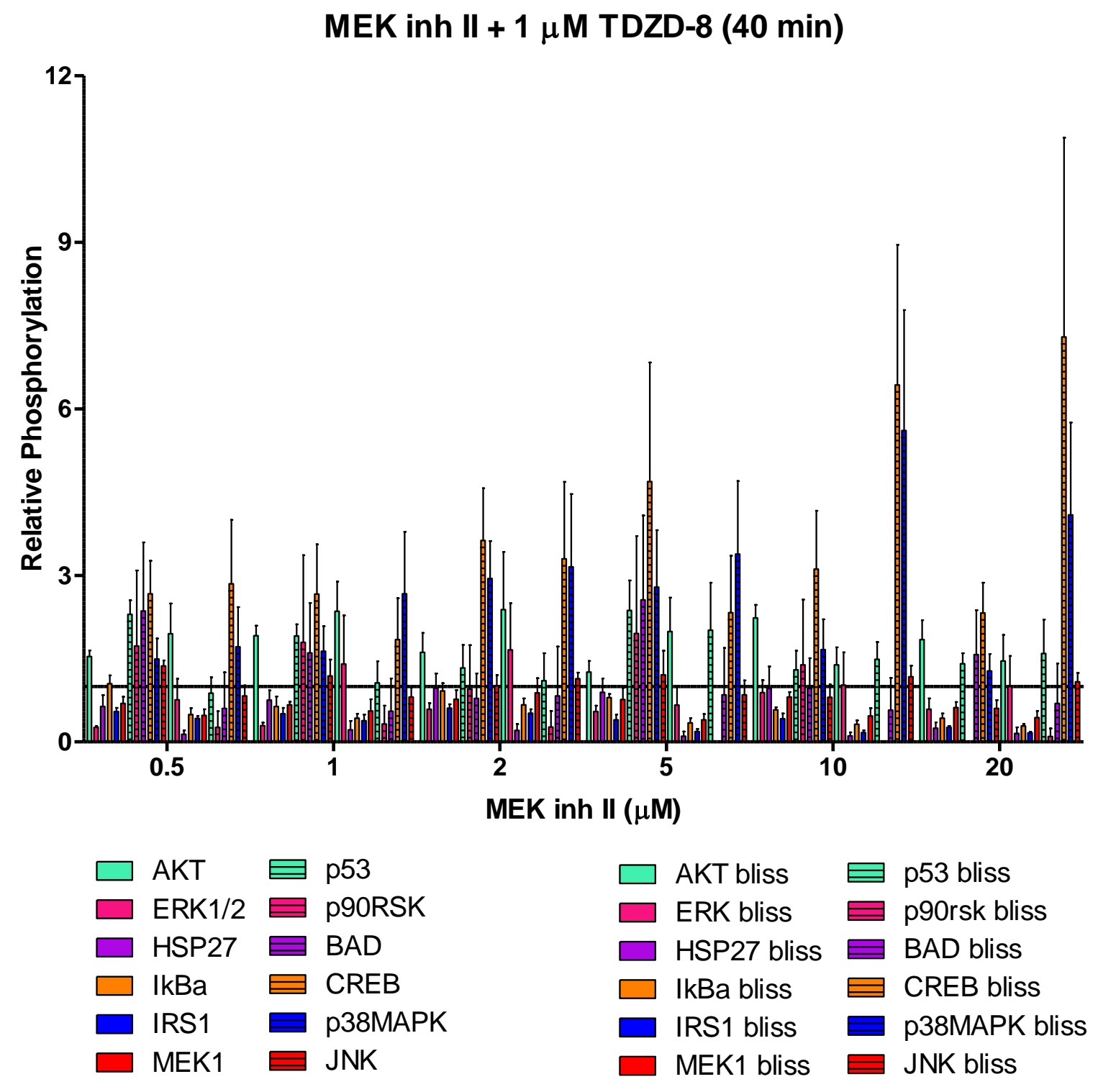


MEK inh II + $5 \mu$ M TDZD-8 (40 min)
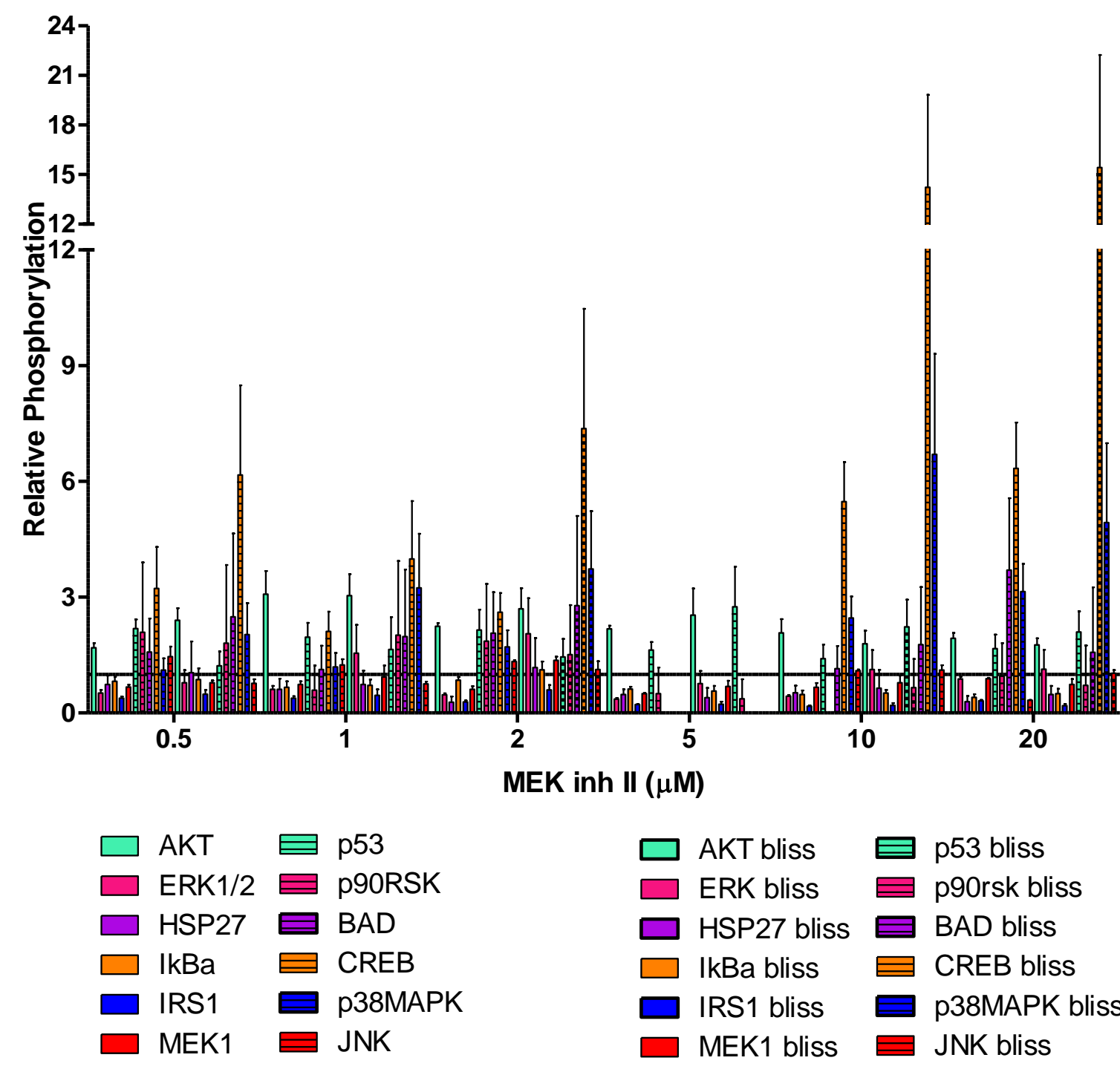


\section{MEK inh II + $10 \mu$ M TDZD-8 (40 min)}

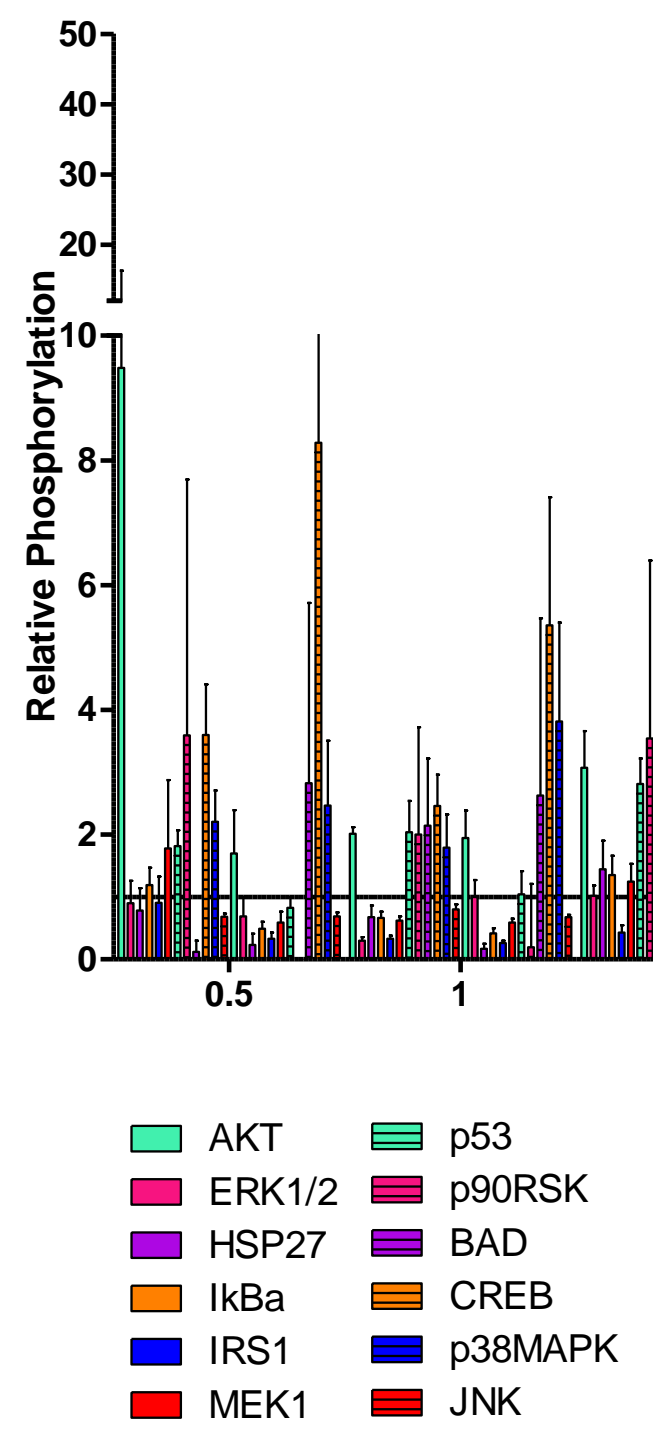




\section{MEK inh II + $20 \mu$ M TDZD-8 (40 min)}

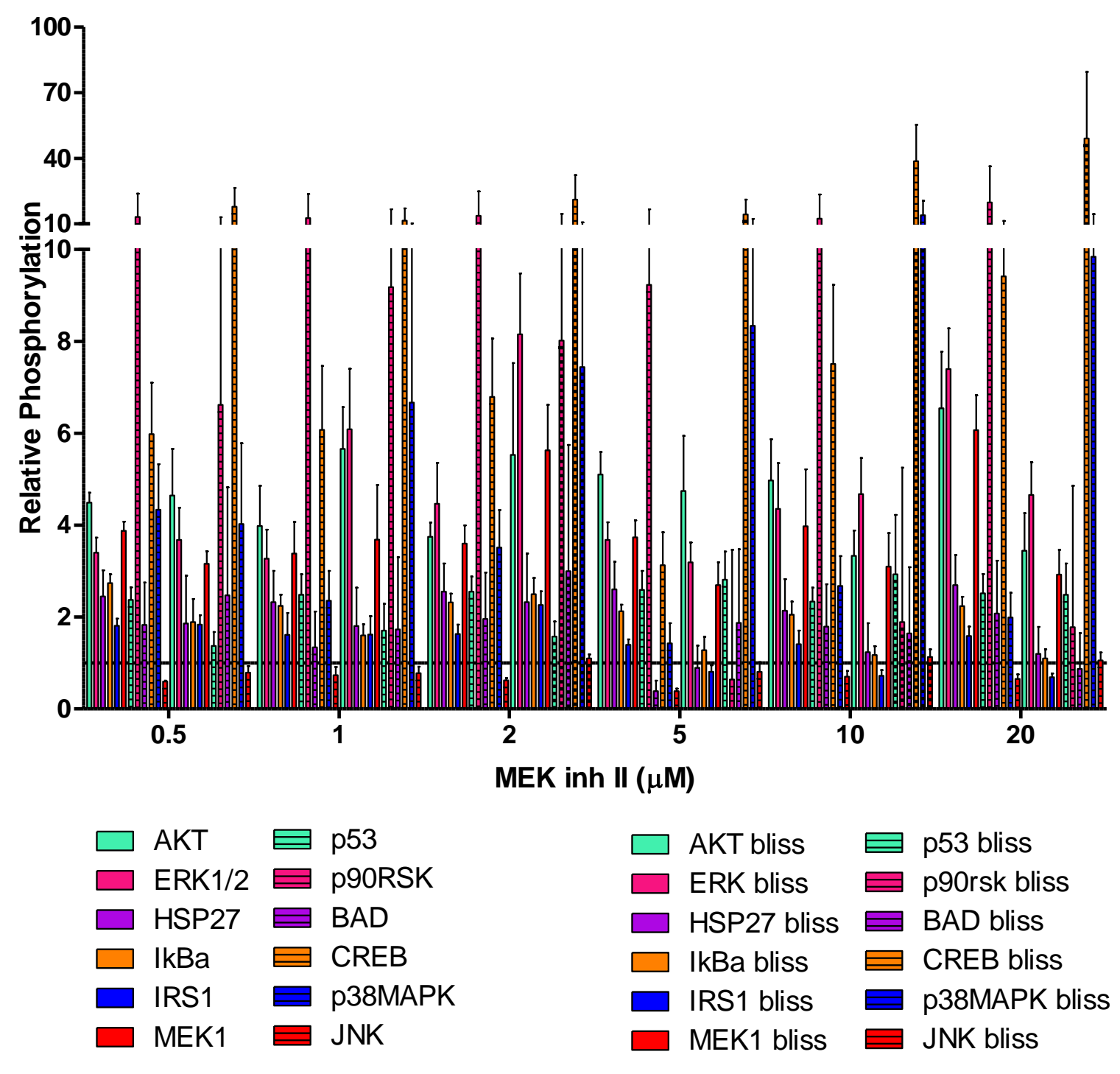


MEK inh II + $50 \mu$ M TDZD-8 (40 min)

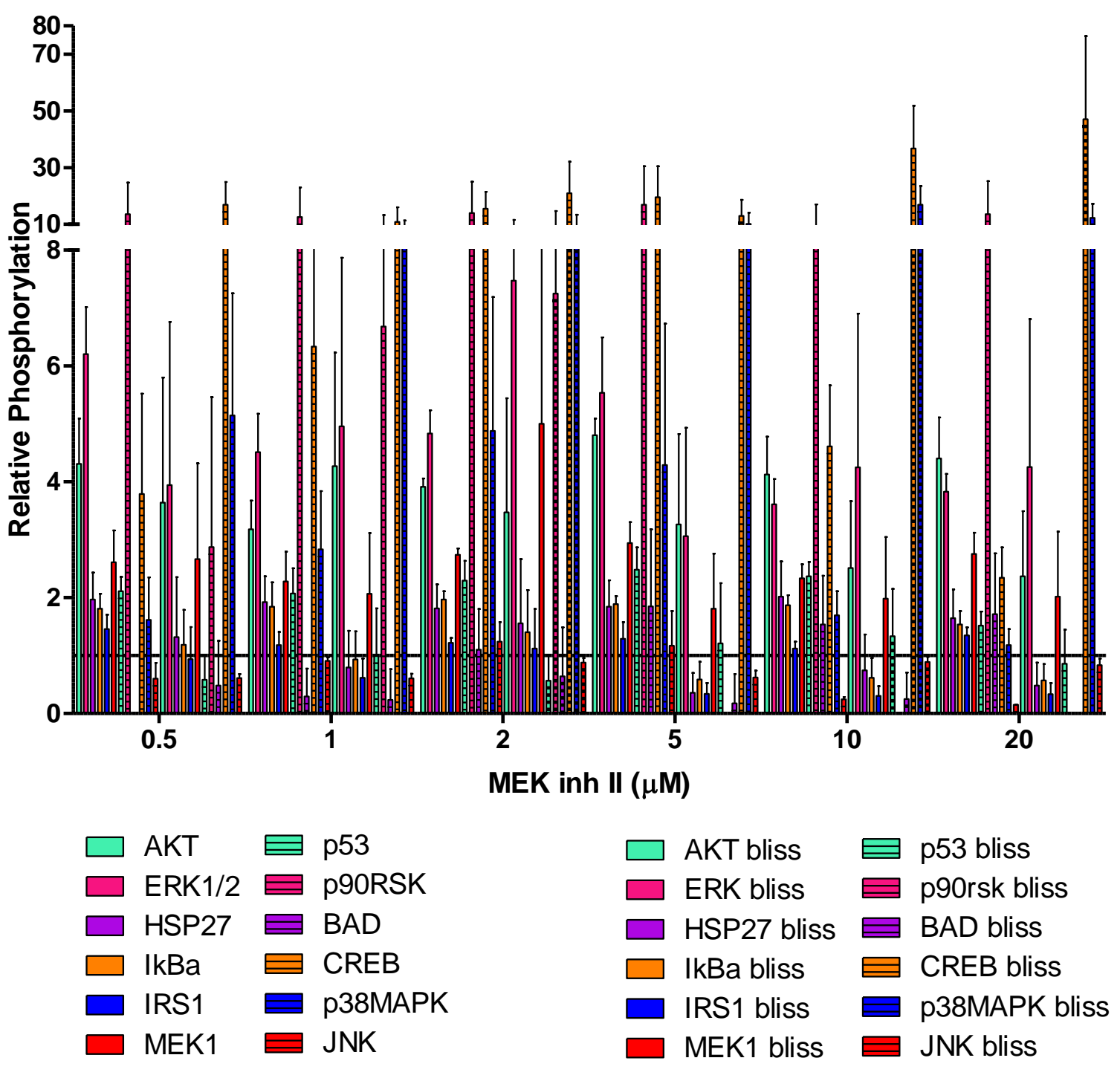



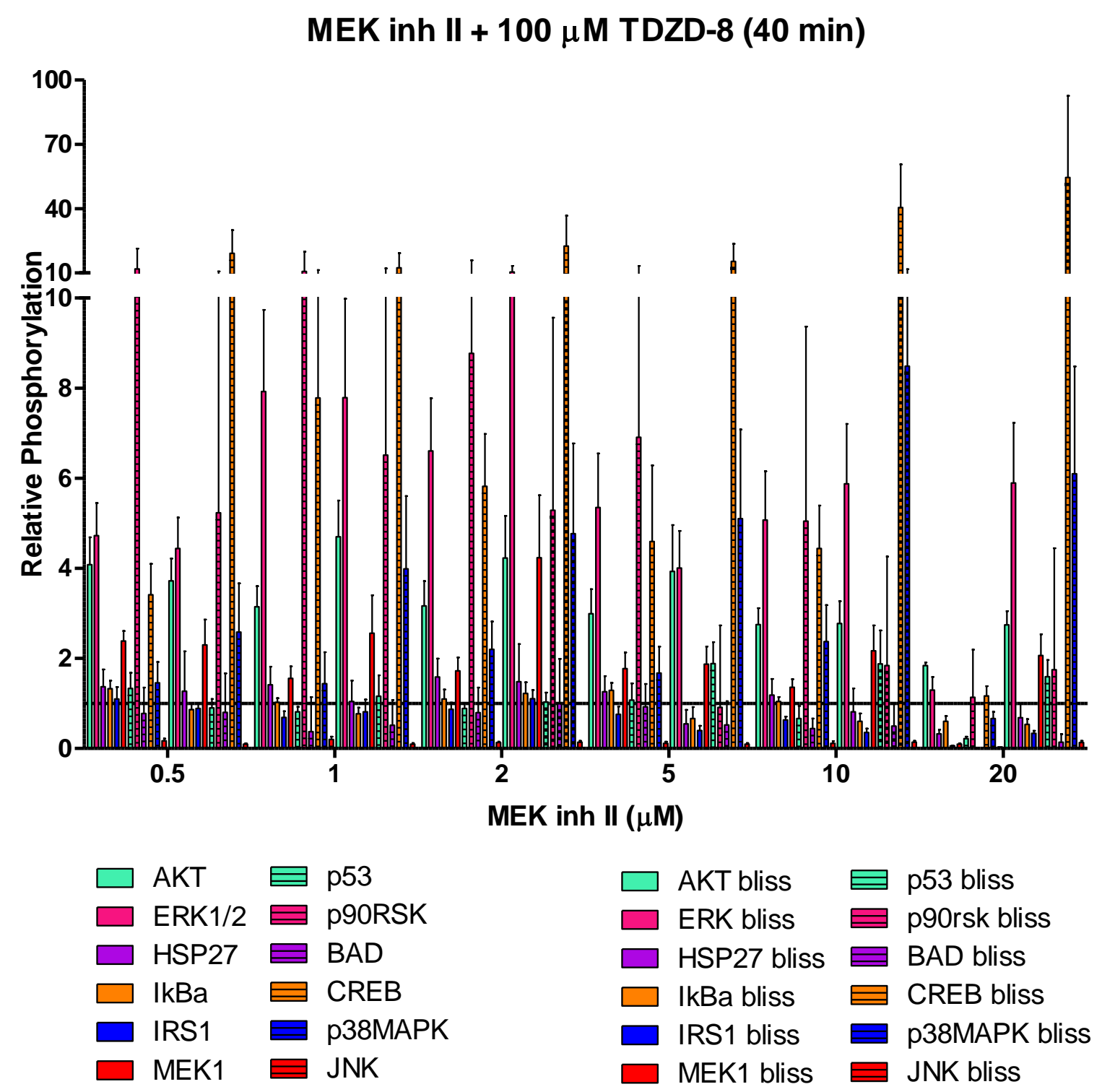
Chapter 4

Using mixtures to ameliorate undesired side-effects of

\author{
deguelin
}




\section{Using mixtures to ameliorate undesired side-effects of deguelin ${ }^{1}$}

The pharmaceutical world has greatly benefited from the well-characterized structure-function relationships of toxins with endogenous biomolecules, such as ionchannels, receptors, and signaling molecules. Thus, therapeutics derived from toxins have been aggressively pursued. However, the multifunctional role of various toxins may lead to undesirable off-target effects, hindering their use as therapeutic agents. In this paper, we suggest that previously unsuccessful toxins (due to off-target effects) may be revisited with mixtures by utilizing the pharmacodynamic response to the potential primary therapeutic as a starting point for finding new targets to ameliorate the unintended responses. In this proof of principle study, the pharmacodynamic response of HepG2 cells to a potential primary therapeutic (deguelin, a plant-derived chemopreventive agent) was monitored, and a possible secondary target (p38MAPK) was identified. As a single agent, deguelin decreased cellular viability at higher doses $(>10 \mu \mathrm{M})$, but inhibited oxygen consumption over a wide dosing range $(1.0-100 \mu \mathrm{M})$. Our results demonstrate that inhibition of oxygen consumption is related to an increase in p38MAPK phosphorylation, and may only be an undesired side effect of deguelin (i.e., one that does not contribute to the decrease in HepG2 viability). We further show that deguelin's negative effect on oxygen consumption can be diminished while maintaining efficacy when used as a therapeutic mixture with the judiciously selected secondary inhibitor (SB202190, p38MAPK inhibitor). These preliminary findings suggest that an endogenous response-directed mixtures approach, which uses a pharmacodynamic

\footnotetext{
${ }^{1}$ Parts of this chapter have been published previously, either in part or in full, from Vrana, J.A., Boggs, N., Currie, H.N., Boyd, J. (2013) Amelioration of an undesired action of deguelin. Toxicon 74, 83-91.

Reproduced with permission from Elsevier.
} 
response to a primary therapeutic to determine a secondary target, allows previously unsuccessful toxins to be revisited as therapeutic mixtures.

\subsection{Introduction}

The well-characterized structure-function relationships of toxins, such as snake venoms and plant-derived toxins, have led to major advances in understanding normal and disease state physiology, as well as the development of pharmacological regimens based on the structures of various toxins (McCleary and Kini, 2012). One such example is captopril, an angiotensin-converting enzyme inhibitor to treat hypertension that is based on bradykinin-potentiating factor (BPF) isolated from the Brazilian pit viper Bothrops jararaca (Ferreira, 1965). Captopril has also been used in combination with marimastat (a matrix metalloproteinase inhibitor) and fragmin (a low molecular weight heparin approved by the U.S. FDA) as an antiangiogenesis therapeutic mixture for patients with advanced stage cancer (Jones et al., 2004). From the successes of captopril, among many other therapeutics derived from toxins, natural toxin-based pharmacology has been aggressively pursued (Koh and Kini, 2012).

Previously known for their success as insecticides and fishing poisons, plantderived rotenoids, such as deguelin, have been investigated for their potential use as chemopreventive (designed to delay the onset of cancer) and chemotherapeutic (designed to destroy cancer after it appears) agents (Kim et al., 2008; Aggarwal et al., 2004). Deguelin, a natural isoflavonoid isolated from the root of Lonchocarpus utilis and Lonchocarpus urucu (Caboni et al., 2004), inhibits NADH:ubiquinone oxidoreductase (complex I) of the mitochondrial electron transport chain (ETC), HSP90, and AKT (Lee 
et al., 2005; Oh et al., 2007, 2008; Peng et al., 2007). As a fishing poison, rotenoids are often used due to their inhibition of cellular respiration via inhibition of complex I of the ETC (Neuwinger, 2004). Inhibition of respiration in cells results in tissue asphyxia and consequently organ paralysis (Neuwinger, 2004). As a chemopreventive agent, rotenoids, specifically deguelin, have shown promise for a variety of cancer types (Chun et al., 2003; Murillo et al., 2002; Peng et al., 2007; Udeani et al., 1997). Unfortunately, when used as a chemotherapeutic, rotenoids have exhibited undesirable side effects, such as respiratory depression and cardiotoxicity, presumably due to a decrease in cellular oxygen consumption caused by inhibition of complex I (Lee, 2004). Additionally, deguelin has been shown to induce a Parkinson's disease-like syndrome in rats when administered in high doses, which is also potentially related to activity at complex I (Caboni et al., 2004). These undesired side effects related to complex I inhibition have hindered deguelin's use as a chemotherapeutic agent (Agarwal and Deep, 2008; Fang and Casida, 1998).

While deguelin has inherent faults that diminish its legitimacy as a chemotherapeutic agent, it may still hold promise as a potential therapeutic if combined with another xenobiotic. In recent years, using a combinatorial/mixtures approach with targeted kinase therapeutics has shown promise as an effective strategy (Engelman et al., 2007; Ma et al., 2005; Namiki et al., 2006; Stommel et al., 2007; Yasui et al., 2007) because many mechanisms for cell survival rely on intricate and sophisticated intracellular signaling networks, making single-point inhibition impractical for treatment (Fitzgerald et al, 2006; Toschi and Janne, 2008). The initial pharmacodynamic response to xenobiotic insult is primarily coordinated by signal transduction networks, which 
typically follow a simple framework: the phosphorylation/dephosphorylation cycle mediated by kinases and phosphatases (Kholodenko, 2006; Sauro and Kholodenko, 2004). Since a typical cellular response to exposure involves the integration of many kinases into pathways for a coordinated response, this enzyme class make advantageous targets for a mixtures approach (Cohen, 2002; Collins and Workman, 2006; Dancey and Sausville, 2003).

There are several different strategies for determining possible mixtures therapies for kinase targets (Jackson, 1993). One strategy involves the simultaneous inhibition of a single target using two or more compounds, while another strategy utilizes two inhibitors to attack two different proteins on a linear pathway (e.g. mitogen-activated protein kinase cascade) (Fitzgerald et al., 2006). A drawback to these mixtures approaches is that prior knowledge of the network and kinases of interest are required to select which inhibitors to use. This paper suggests an alternative strategy: instead of selecting mixtures based on previously known mechanisms and complex networks that may not fit the cell line or disease of interest, the endogenous response of the cell to a primary therapeutic may be used to select the secondary target of interest. By monitoring the endogenous response of the network while under duress from a primary therapeutic, the cell's alternate mode of survival can be exposed, and a second inhibitor may be selected for a more effective mixtures therapeutic strategy. By utilizing the endogenous response to primary therapeutic, undesirable effects (such as decreased oxygen consumption following exposure to deguelin) may be targeted for amelioration. Overall, this strategy holds promise for potentially maintaining efficacy of an initial therapeutic (deguelin) while reducing side effects for improved patient outcome. 
In this study, we investigated the endogenous response of HepG2 cells, a human hepatocellular carcinoma-derived cell line, to a primary therapeutic (deguelin) at varying doses to determine a potential secondary target for a beneficial therapeutic mixture. We selected the HepG2 cell line as our model in vitro system due to the central role that the liver plays in xenobiotic biotransformation after exposure (Mersch-Sundermann, et al., 2004). Most importantly, the HepG2 cell line retains endogenous xenobiotic metabolizing enzymes, whereas primary hepatocyte culture typically loses these vital enzymes (Knasmuller, et al., 1998). We monitored the response of HepG2 cells to deguelin by determining 24 hour viability and kinetically measuring oxygen consumption over 24 hours. From this data, we found a critical shift in oxygen consumption at 400 minutes post-dose that spanned several doses. At this time-point, we monitored the relative post-translational phosphorylation of 8 proteins involved in cell proliferation or apoptotic signal transduction cascades. From this phosphorylation response data, we selected p38MAPK as a secondary target, and used a specific p38MAPK inhibitor (SB202190) in combination with deguelin to possibly alter the overall cellular response to deguelin. We found that by using an endogenously selected therapeutic mixture, deguelin's inhibition of oxygen consumption was diminished while maintaining efficacy. This proof of principle study shows that by using an endogenous response-directed mixtures approach, previously unsuccessful toxins as a primary therapeutic may be revisited. 


\subsection{Materials and Methods}

\subsubsection{Materials}

Deguelin (CAS no. 522-17-8) and SB202190 (CAS no. 152121-30-7) were obtained from Sigma Aldrich (St. Louis, MO). RPMI-1640 containing phenol red, RPMI-1640 without phenol red, sodium pyruvate, HEPES, L-glutamine, fetal bovine serum, and penicillin-streptomycin were obtained from Invitrogen (Carlsbad, CA). Cell lines and MTT assay kits were obtained from American Type Culture Collection (Manassas, VA). MitoXpress oxygen probe was obtained from Luxcel Corporation (Cork, Ireland). BioPlex beads, lysis buffer, and reagents necessary for determination of relative phosphorylation were obtained from BioRad (Hercules, CA).

\subsubsection{Cell culture}

Human hepatocellular carcinoma-derived HepG2 cells were cultured in RPMI1640, supplemented with $1 \mathrm{mM}$ sodium pyruvate, $5 \mathrm{mM}$ HEPES, $2 \mathrm{mM}$ L-Glutamine, $10 \%$ fetal bovine serum, $100 \mathrm{U} / \mathrm{ml}$ penicillin, and $100 \mu \mathrm{g} / \mathrm{ml}$ streptomycin. Cells were maintained in a humidified atmosphere at $37^{\circ} \mathrm{C}, 5 \% \mathrm{CO} 2$ and passaged at $80 \%$ confluence. 


\subsubsection{Dosimetry}

Cells were seeded into clear-bottom, black-sided 96-well plates at a concentration of $4 \times 10^{4}$ cells per well in RPMI-1640 without phenol red and allowed to grow for 24 hours before dosing. Media was then aspirated from wells and cells were challenged with varying doses of single and mixed compounds in fresh media. Compounds were prepared so that resulting well concentrations would be $<1 \%$ DMSO and $0.01 \mu \mathrm{M}$ to 100 $\mu \mathrm{M}$ (deguelin) and $350 \mathrm{nM}(\mathrm{SB} 202190)$.

\subsubsection{MTT assay}

After 24 hours of exposure to single compounds or mixtures, cell viability was determined using the MTT (3-(4,5-dimethyl)-2,5-diphenyl tetrazolium bromide) assay, according to the manufacturer's protocol. The assay is based on the reduction of tetrazolium MTT to formazan by metabolically active cells, in part by the action of dehydrogenase enzymes, to generate reducing equivalents such as NADH and NADPH. Briefly, MTT reagent was added to the wells of the microplate, and after two hours of incubation at $37^{\circ} \mathrm{C}$, intracellular formazan crystals were solubilized with the provided detergent solution. Absorbance values were obtained using the Safire2 microplate reader (Tecan US, Raleigh, NC) with a measurement wavelength of $570 \mathrm{~nm}$ and a reference wavelength of $700 \mathrm{~nm}$, read from the bottom. Experiments were each performed at least in triplicate. Percent viability was calculated by normalizing to controls, which received dosing vehicle (1\% DMSO). 


\subsubsection{Oxygen consumption assay}

Immediately after dosing with single compounds or mixtures, cellular oxygen consumption was assessed using the MitoXpress probe, according to manufacturer's protocol. Briefly, oxygen-sensitive probe was diluted to a stock concentration of $1 \mu \mathrm{M}$, and stock probe was diluted 1:15 in each well of a 96-well plate containing cells; $100 \mu \mathrm{L}$ of pre-warmed mineral oil was also added to each well to block ambient oxygen from the cells. After pre-warming the plates at $37^{\circ} \mathrm{C}$ for 1 hour, cells were challenged with varying doses of deguelin alone or in combination the IC50 of SB202190 (350nM). Immediately following addition of compound(s), oxygen consumption was determined by measuring fluorescence. Fluorescent signal was obtained using the Infinite M1000 microplate reader (Tecan US, Raleigh NC) with excitation wavelength of $380 \mathrm{~nm}$ and emission wavelength of $650 \mathrm{~nm}$, reading from the bottom every 10 minutes for 24 hours after dosing. Experiments were performed in quadruplicate. Relative oxygen consumption was calculated by normalizing to controls, which received dosing vehicle (1\% DMSO).

\subsubsection{Bio-Plex Multiplex Immunoassay}

After 400 minutes of exposure (Boyd et al., 2012) to increasing doses of deguelin $(0.01,0.1,1.0,10,100 \mu \mathrm{M})$ in $1 \%$ DMSO alone, or in combination with IC50 of SB202190 (350nM), cells were lysed using lysis buffer (BioRad, Hercules, CA) with $500 \mu \mathrm{M}$ phenylmethanesulfonylfluoride (PMSF) (Sigma, St. Louis, MO) and phosphatase inhibitors (BioRad, Hercules, CA). Total protein concentration was determined using the 
DC Protein Assay (BioRad, Hercules, CA) according to the manufacturer's instructions. Protein phosphorylation was detected using multiplex bead-based BioPlex suspension array system (Bio-Rad, Hercules, CA) and lysates were prepared according to the manufacturer's protocol. Beads and detection antibodies against phosphorylated ERK1/2 (Thr202/Tyr204, Thr185/Tyr187), AKT (Ser473), HSP27 (Ser78), IкB $\alpha$ (Ser32/Ser36), JNK (Thr183/Tyr185), p38MAPK (Thr180/Tyr182), p53 (Ser15), and p90RSK (Thr359/Ser363) were obtained from Bio-Rad (Hercules, CA). Relative phosphorylation was calculated by normalizing to control cells, which received dosing vehicle (1\% DMSO). All experiments were performed in duplicate.

\subsubsection{Statistical Analysis}

Dose-response curves for MTT assays were generated by best-fit Hill-plot regression of scatter plot data using Prism V5 (Graphpad Software, San Diego, CA). Oxygen consumption curves were generated by choosing the best-fit polynomial regression of scatter plot data using Prism V5 (Graphpad Software, San Diego, CA). The time point of interest (400 min) was selected using SAS JMP V8 (Cary, NC) where the change in the slope of the oxygen consumption curve reached a minimum for most exposures. Statistical significance for viability and relative phosphorylation was assessed by using a two-way analysis of variance (ANOVA) with Bonferroni post-test. Statistical significance for oxygen consumption (a kinetic assay) was assessed by using a KruskalWallis nonparametric test with Dunns post-test. A difference at $\mathrm{P}<0.05$ level was considered statistically significant. For viability and relative phosphorylation data, error 
bars reflect standard error of the mean. For oxygen consumption curves (best-fit polynomial regression), error was reported at the $95 \%$ confidence interval.

\subsection{Results}

\subsubsection{Deguelin as a monotherapy}

To determine the overall effect of deguelin alone, HepG2 cells were exposed to $\log$ doses of deguelin $(0.01-100 \mu \mathrm{M})$ and viability at 24 hours post-dose was measured using the MTT assay (shown in Figure 4.1). Our viability data suggests that deguelin is effective at doses greater than $10 \mu \mathrm{M}\left(\mathrm{EC}_{50}=45 \pm 1 \mu \mathrm{M}\right)$.

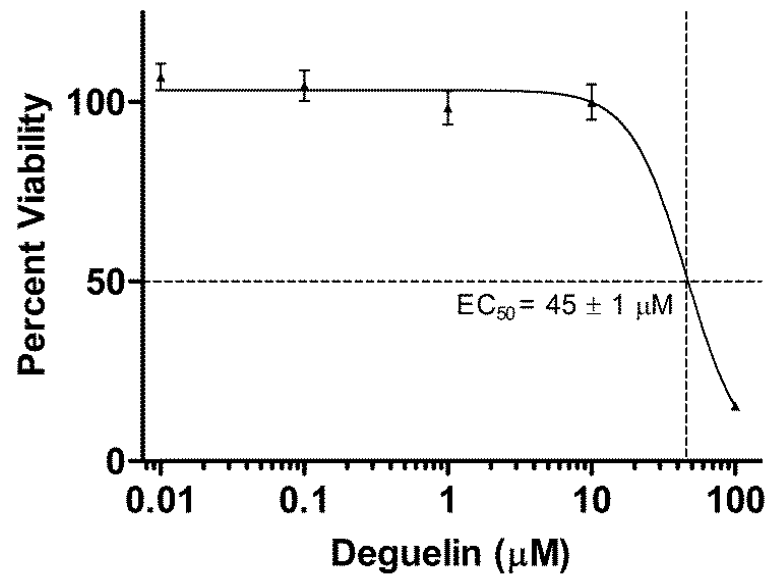

Figure 4.1. Percent viability of HepG2 cells in response to 24 hour exposure of $0.01-100 \mu M$ deguelin. Viability, shown as \% viability, was measured as in Methods and was calculated relative to control cells which received dosing vehicle $(<1 \%$ DMSO) only. Dose-response curve was generated by a best-fit Hill-plot regression of scatter plot data. Error is reported as \pm standard error of the mean (SEM). 
4.3.2 HepG2 viability (in response to deguelin) may not be directly associated with $\mathrm{O}_{2}$ consumption

To better relate deguelin's inhibition of oxygen consumption to endpoint viability, we kinetically measured the oxygen consumption of HepG2 cells in response to deguelin over 24 hours post-dose, as shown in Figure 4.2, using the MitoXpress oxygen consumption assay. From Figure 4.2, oxygen consumption decreased when in the presence of 1.0 $-100 \mu \mathrm{M}$ doses of deguelin (as

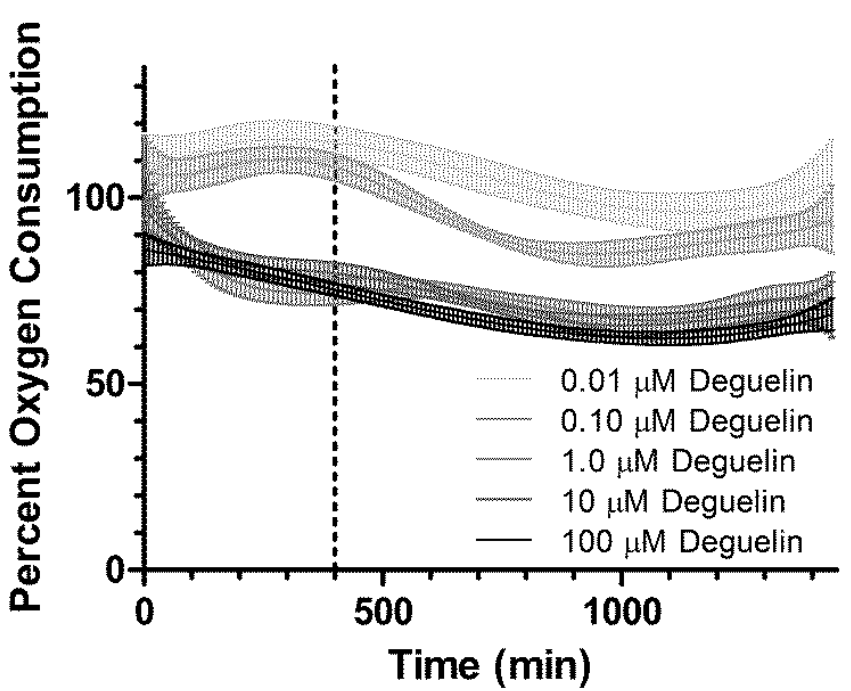

Figure 4.2. Percent oxygen consumption of HepG2 cells in response to $0.01-100 \mu \mathrm{M}$ deguelin. Oxygen consumption response of HepG2 cells to increasing doses of deguelin (shown in shades of gray) measured over 24 hours. Oxygen consumption was measured using MitoXpress extracellular assay as outlined in section 2.5. Percent oxygen consumption is shown relative to control cells which received dosing vehicle ( $<1 \%$ DMSO) only. Oxygen consumption curves were generated using a bestfit polynomial function with error bars reflecting the $95 \%$ confidence intervals. Vertical line at 400 minutes was selected as the time-point of greatest disparity in oxygen consumption.

compared to controls, represented as percent oxygen consumption). A Kruskal-Wallis nonparametric test with Dunns post-test was used to compare oxygen consumption responses to various doses. The oxygen consumption responses at all doses were statistically different $(\mathrm{P}<0.05)$ from each other except for $1.0 \mu \mathrm{M}$ vs $10 \mu \mathrm{M}$ and $10 \mu \mathrm{M}$ vs $100 \mu \mathrm{M}$. Most importantly, there is a clear separation in oxygen consumption responses between two dosing groups: the lowest doses $(0.01$ and $0.1 \mu \mathrm{M})$ and the higher doses $(1.0-100 \mu \mathrm{M})$. Since our oxygen consumption data at these doses does not seem to correspond with our MTT assay (which demonstrated no decrease in viability for the 1.0 and $10 \mu \mathrm{M}$ doses), the inhibition of oxygen consumption may not be directly 
associated with 24 hour HepG2 viability in response to deguelin. Furthermore, for the lower doses of deguelin $(0.01$ and $0.1 \mu \mathrm{M})$, oxygen consumption actually increases relative to controls until about 400 minutes post-exposure; at this time-point, oxygen consumption decreases, ultimately returning to control levels. Therefore, 400 minutes appears to be an interesting time point post-exposure that results in the greatest disparity in oxygen consumption between high and low doses. Since the full dosing regimen of deguelin indicated 400 minutes post-dose as a key time-point where doses of deguelin lead to both decreased $(1.0-100 \mu \mathrm{M})$ and increased $(0.01,0.1 \mu \mathrm{M})$ oxygen consumption, we investigated the post-translational phosphorylation response to identify the underlying signal transduction events that allow for both decreased oxygen consumption and survival (ie. $\sim 100 \%$ viability) at the 1.0 and $10 \mu \mathrm{M}$ doses. This method of selecting critical time-points of phosphorylation events from oxygen consumption data has been previously used by Boyd et al. (2012).

\subsubsection{Endogenous phosphorylation response to deguelin exposes a potential secondary} therapeutic target

We next explored the signal transduction response, by means of post-translational phosphorylation activity, of HepG2 cells exposed to increasing doses of deguelin. We simultaneously measured the phosphorylation of ERK1/2 (Thr202/Tyr204, Thr185/Tyr187), AKT (Ser473), HSP27 (Ser78), IкB $\alpha \quad$ (Ser32/Ser36), JNK (Thr183/Tyr185), p38MAPK (Thr180/Tyr182), p53 (Ser15), and p90RSK (Thr359/Ser363) at 400 minutes post-exposure to deguelin using bead-based ELISA flow cytometry. These protein targets were selected because of their relevance in signal 
transduction pathways related to cellular death and recovery [Dong et al., 2002; Jin and El-Deiry, 2005; Oren, 2003; Wilkinson and Millar, 2000], and due to the availability of selective inhibitors. Figure 4.3 shows the relative phosphorylation response of HepG2 cells to 400 minute exposures of increasing doses of deguelin (as compared to controls, normalized to a value of 1). A two-way ANOVA with Bonferroni post-test determined significant differences $(\mathrm{P}<0.05)$ between deguelin-exposed and control cells for p38MAPK at $0.1 \mu \mathrm{M}$; ERK1/2, JNK, p38MAPK, IкB $\alpha$, p53, and p90RSK at $1.0 \mu \mathrm{M}$; for all proteins except JNK at $10 \mu \mathrm{M}$; and for all proteins at $100 \mu \mathrm{M}$ (Figure 4.3). While several proteins experienced a relative phosphorylation change in response to deguelin at 400 minutes post-dose, p38MAPK phosphorylation is statistically different across four orders of magnitude of dose $(1.0-100 \mu \mathrm{M})$.

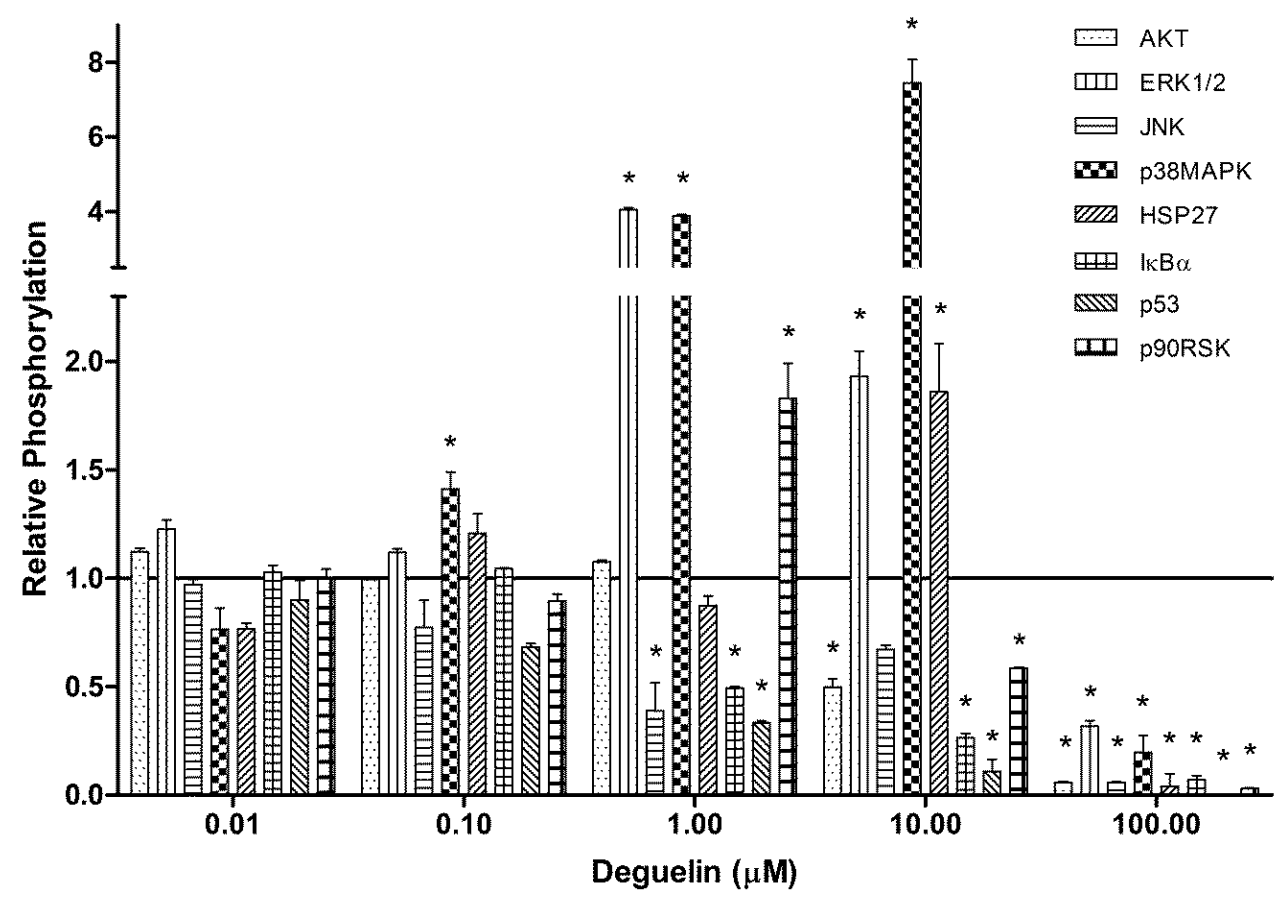

Figure 4.3. Relative phosphorylation of protein targets in response to $0.01-100 \mu \mathrm{M}$ deguelin. Posttranslational phosphorylation response of protein targets (from cell lysates) to 400 minute exposures of deguelin as outlined in Methods; responses are shown relative to control cells, which received dosing vehicle ( $<1 \%$ DMSO), but no deguelin. The solid black line at $\mathrm{y}=1$ shows where observed post-translational phosphorylation responses are the same as control. The phosphorylation responses found to be significantly different $(\mathrm{P}<0.05)$ from controls are marked with *. 


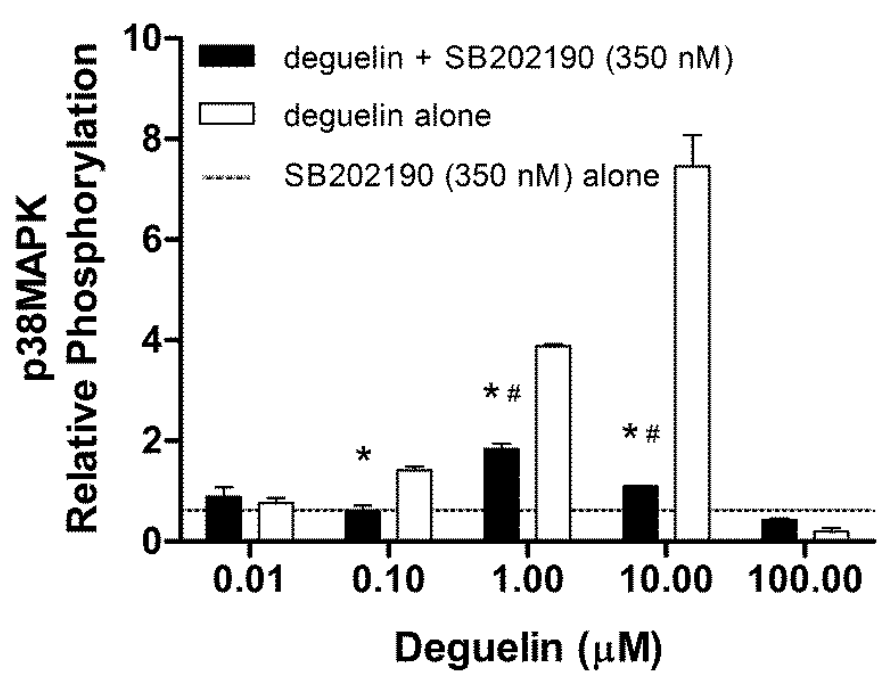

Figure 4.4. Relative p38MAPK phosphorylation in response to deguelin, SB202190, and mixtures of deguelin with SB202190. The phosphorylation response of p38MAPK from cell lysates at 400 minute exposures to mixtures of deguelin and $350 \mathrm{nM} \mathrm{SB} 202190$ (black), deguelin alone (white), and 350 nM SB202190 alone (black dots with grey line representing standard error of the mean) as discussed in sections 2.6 and 3.4; all values are reported as relative to control cells, which received dosing vehicle ( $<1 \%$ DMSO) only. Error is reported as \pm SEM. Post-translational phosphorylation responses of the mixture found to be significantly different $(\mathrm{P}<0.05)$ from deguelin alone are marked with $*$ and those different $(\mathrm{P}<0.05)$ than SB202190 alone are marked with \#.

\author{
4.3.4 Using mixtures to suppress \\ endogenous p38MAPK response to \\ deguelin
}

To determine if our directed mixtures approach, which takes advantage of the endogenous pharmacodynamic response to deguelin, has the potential to decrease viability while minimizing deguelin's effect on oxygen consumption, we attempted to suppress the post-translational p38MAPK phosphorylation response to deguelin by using a

secondary inhibitor, SB202190. We chose SB202190 because it is a selective inhibitor of p38MAPK (Lee et al., 1994). HepG2 cells were exposed to SB202190 alone at its manufacturer reported $\mathrm{IC}_{50}(350 \mathrm{nM})$ and in combination with a full range of deguelin doses $(0.01-100 \mu \mathrm{M})$, and we measured the p38MAPK post-translational phosphorylation response at 400 minutes post-dose (shown in Figure 4.4). From this figure, SB202190 alone does decrease the phosphorylation response of p38MAPK $(0.61$ $\pm 0.02)$ when compared to controls, indicating that it is a relatively potent inhibitor (350 nM) of p38MAPK. A two-way ANOVA with Bonferroni post-test determined significant differences $(\mathrm{P}<0.05)$ between the $\mathrm{p} 38 \mathrm{MAPK}$ response to deguelin alone and 
the mixture, as well as SB202190 alone and the mixture. Most notably the relative p38MAPK phosphorylation responses to mixtures of deguelin $(0.1-10 \mu \mathrm{M})$ with SB202190 were significantly decreased in comparison to deguelin alone.

4.3.5 Mixtures approach retains efficacy while decreasing deguelin's effect on $\mathrm{O}_{2}$ consumption

To test this new potential therapeutic regimen, we exposed HepG2 cells to varying doses of deguelin $(0.01-100 \mu \mathrm{M})$ in combination with SB202190 at its

manufacturer reported $\mathrm{IC}_{50} \quad(350$

$\mathrm{nM})$ and measured viability (Figure

4.5). At 24 hours post-dose, viability was measured following treatment with SB202190 alone and in combination with deguelin (Figure 4.5), and compared to viability data for deguelin alone (controls measured concurrently). SB202190 alone did not significantly decrease viability at the $350 \mathrm{nM}$ dose $(98 \pm 3 \%)$. Viability was slightly decreased in response to mixtures of deguelin

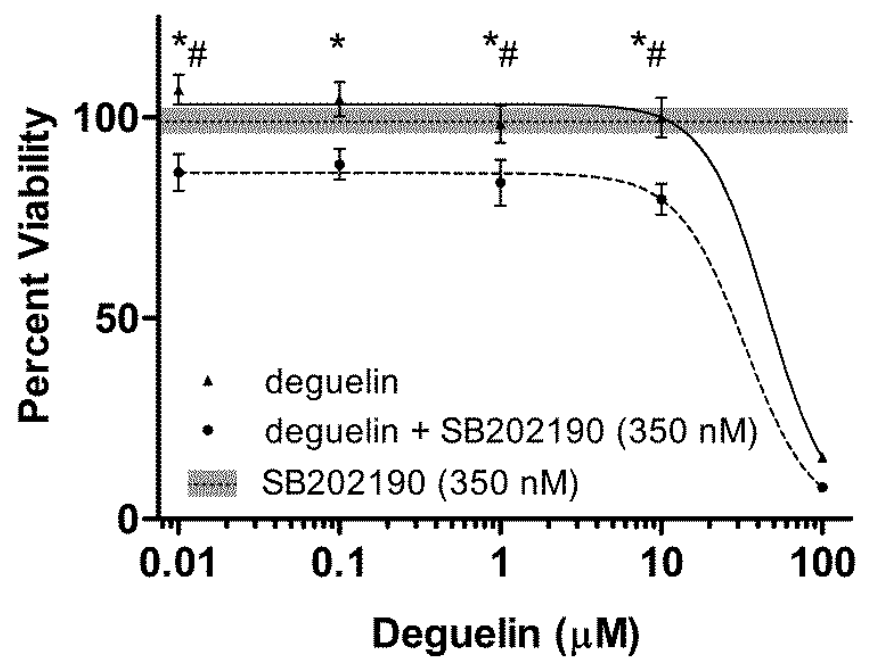

Figure 4.5. Percent viability of HepG2 cells in response to 24 hour exposure of deguelin alone and in combination with SB202190. HepG2 viability in response to deguelin alone (triangles), mixtures of deguelin and $350 \mathrm{nM} \mathrm{SB} 202190$ (circles), and $350 \mathrm{nM}$ SB202190 alone (black dotted line with grey shading for SEM). Viability, shown as \% viability, was measured as outlined in section 2.4, and was calculated relative to control cells which received dosing vehicle ( $<1 \%$ DMSO), but no deguelin or inhibitor doses. Dose-response curves were generated by best-fit Hillplot regression of scatter plot data. Mixture viability responses found to be significantly different $(\mathrm{P}<0.05)$ from deguelin are marked with $*$ and those different $(\mathrm{P}$ $<0.05)$ from SB202190 are marked with \#. 
$\left(\mathrm{EC}_{50}=33 \pm 1 \mu \mathrm{M}\right)$ when compared to deguelin alone $\left(\mathrm{EC}_{50}=45 \pm 1 \mu \mathrm{M}\right)$. To determine the effect of this mixtures technique on oxygen consumption, HepG2 cells were exposed to a full dosing regimen of deguelin $(0.01-100 \mu \mathrm{M})$ in combination with SB202190 (350 nM). Immediately after dosing, oxygen consumption was measured kinetically over 24 hours and compared relative to controls, shown as percent oxygen consumption. From Figure 4.6, SB202190 alone did not affect oxygen consumption from 0 - 600 minutes post-dose.

Figure 4.6. Percent oxygen consumption of
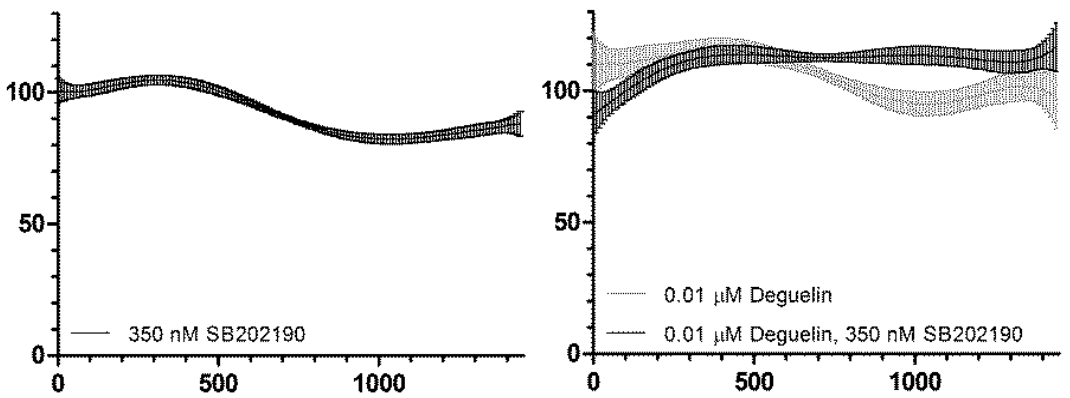
HepG2 cells in response to deguelin alone, and in combination with SB202190. Oxygen consumption response of HepG2 cells to SB202190 alone (squares), deguelin alone (triangles) and deguelin with 350 nM SB202190 (circles) measured over
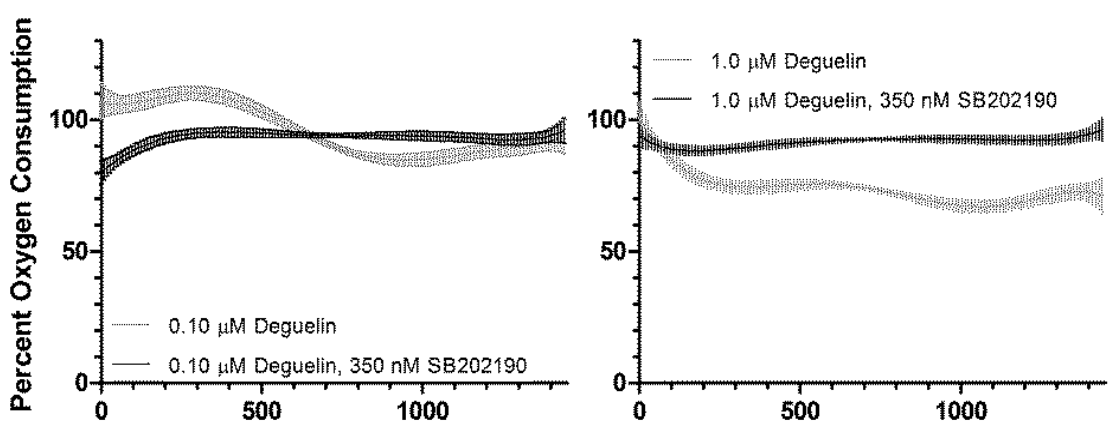
24 hours. Oxygen consumption was measured using MitoXpress extracellular assay as outlined in section 2.5. Oxygen consumption is shown relative to control cells, which received dosing vehicle (<1\% DMSO), but no deguelin or
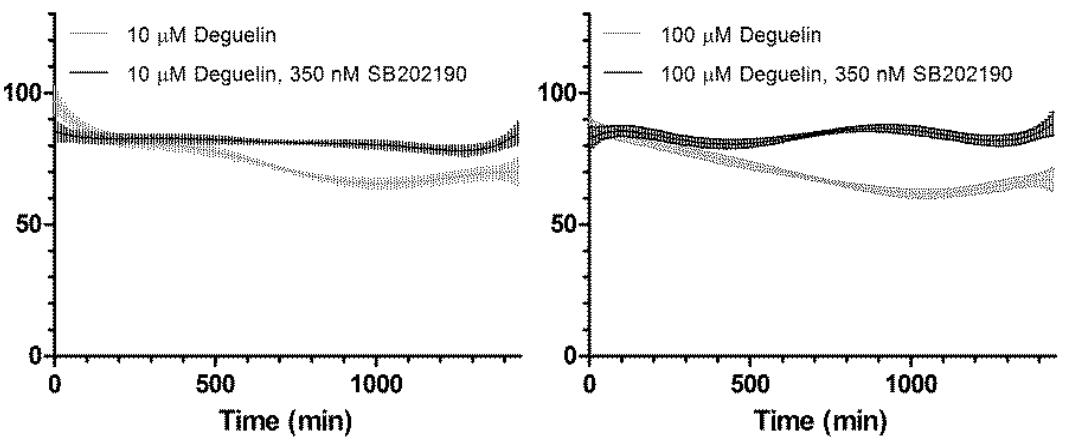
SB202190. Oxygen consumption curves were generated using a best-fit polynomial function (solid line) with surrounding dotted lines reflecting the $95 \%$ confidence intervals.

However, after 600 minutes SB202190 decreased oxygen consumption. Mixtures of deguelin $(0.01-100 \mu \mathrm{M})$ with $350 \mathrm{nM} \mathrm{SB} 202190$ sustained oxygen consumption (100\% 
oxygen consumption relative to controls) for $0.01 \mu \mathrm{M}$ and $0.1 \mu \mathrm{M}$, or increased oxygen consumption $(1.0-100 \mu \mathrm{M})$, whereas exposure to deguelin alone inhibited oxygen consumption at doses of $1.0-100 \mu \mathrm{M}$. A Kruskal-Wallis nonparametric test with Dunns post-test was used to compare oxygen consumption responses between doses of deguelin alone and in combination with SB202190. When compared to oxygen consumption in response to deguelin alone, mixture doses were statistically different $(\mathrm{P}<0.05)$ for 1.0 , 10 and $100 \mu \mathrm{M}$. When considering viability, efficacy of the highest mixture dose was sustained, while oxygen consumption at the highest mixture dose of deguelin $(100 \mu \mathrm{M})$ with SB202190 was significantly increased $(\mathrm{P}<0.05)$ when compared to $100 \mu \mathrm{M}$ deguelin alone. By increasing oxygen consumption using this endogenously-directed mixtures approach, we were able to successfully ameliorate an undesired alternative action of deguelin.

\subsection{Discussion}

Due to the activity of various toxins at specific intracellular proteins, therapeutic agents derived from these toxins have been aggressively pursued (McCleary and Kini, 2012). One such example is cobrotoxin, a protein isolated from the venom of the Taiwan cobra Naja naja atra (Chang et al., 1997). Cobrotoxin binds to nuclear factor- $\mathrm{B}$ (NF$\kappa \mathrm{B})$ signaling molecules with high affinity, such as p50 and inhibitory $\kappa \mathrm{B}$ kinases (IKKs) (Park et al., 2005). Therapeutic agents capable of suppressing NF- $\kappa \mathrm{B}$ are constantly being explored due to abnormal or constitutive NF- $\kappa \mathrm{B}$ activity in many cancer types (Dolcet et al., 2005). Cobrotoxin's inhibition of NF- $\kappa B$ signaling molecules suppresses NF- $\kappa \mathrm{B}$ activity, making cobrotoxin an advantageous chemotherapeutic agent (Son et al., 
2007). From this ideology, the present proof of principle study has demonstrated that an endogenous response-directed mixtures approach may be useful for diminishing possible off-target effects while maintaining efficacy of naturally-derived potential therapeutics. Deguelin, a plant-derived chemopreventive agent, has been shown (Figure 4.1) to decrease HepG2 viability at high doses. Previously, deguelin has been shown to alter activity at different intracellular proteins, such as AKT and HSP90 (Lee et al., 2005; Oh et al., 2007, 2008; Peng et al., 2007), making it a valid candidate for kinase-targeted chemotherapy. However, deguelin has also been shown to affect mitochondrial bioenergetics, presumably due to its activity at complex I of the electron transport chain (Fang and Casida, 1998;Gerhauser, et al., 1997). Previously, this inhibitory activity has been shown to decrease intracellular oxygen consumption in a mouse skin model (Gerhauser, et al., 1997), which may also contribute to cellular toxicity at distal cells. Our results (Figure 4.2) indicate that off-target inhibition of oxygen consumption was sustained over 24 hours in response to a range of doses $(1.0-100 \mu \mathrm{M})$ of deguelin when it was used as a monotherapy. While inhibition of oxygen consumption was sustained over a wide dosing range, a significant decrease in HepG2 viability occurs only at doses greater than $10 \mu \mathrm{M}$. This suggests that deguelin's inhibition of oxygen consumption may not be contributing to the decrease in HepG2 viability. It is known from the Warburg Effect that cancer cells can utilize aerobic glycolysis for ATP production, whereas differentiated cells primarily produce ATP via oxidative phosphorylation that requires sufficient amounts of oxygen (Warburg, 1956). In the absence of oxygen, it is difficult for differentiated cells to produce enough ATP from anaerobic glycolysis for survival, thus many undergo apoptosis under hypoxic conditions. However, cancer cells, such as 
HepG2, can survive in an oxygen-deficient environment due to their reliance on aerobic glycolysis to produce ATP [for a review, see Vander Heiden et al., 2009]. Therefore, the Warburg Effect, in conjunction with our results, supports that deguelin's activity at separate intracellular sites may be causing decreased viability, whereas inhibition of oxygen consumption is possibly an undesirable alternative activity of deguelin that limits its usefulness as a possible therapeutic (Lee et al., 2005; Oh et al., 2007, 2008).

To better understand the discrepancies in HepG2 viability and oxygen consumption in response to deguelin, we investigated the post-translational phosphorylation response of several key proteins involved in cell recovery or proapoptotic signaling cascades (Figure 4.3). We found that p38MAPK phosphorylation in response to deguelin is significantly different over several orders of magnitude ( $0.1-100$ $\mu \mathrm{M})$ of dose. For $0.1-10 \mu \mathrm{M}$ doses of deguelin, the relative phosphorylation response of p38MAPK is significantly increased. With regard to HepG2 viability, the $0.1-10 \mu \mathrm{M}$ dosing range is ineffective, indicating p38MAPK as a possible protein involved in an endogenous defense mechanism that improves survival against deguelin. This is further supported by the decrease in relative phosphorylation of p38MAPK in response to 100 $\mu \mathrm{M}$ dose of deguelin that coincides with decreased viability at this dose, indicating the cell may not be able to effectively respond to a high dose of deguelin. This preliminary data suggests that a directed mixtures approach utilizing p38MAPK as a secondary target, thereby capitalizing on the endogenous response to deguelin, may be advantageous. However, it should be noted that due to the limited number of proteins measured in this study, there may be activity at other proteins/cascades that could be influencing the overall pharmacodynamic response to deguelin. 
While monotherapies for cancer have proven to be successful when a single target controls the cell's fate, many carcinomas are often more complex, with intricate signaling networks governing proliferation (Toschi and Janne, 2008). This complex survival framework, capable of facilitating uncontrolled proliferation, can withstand the inhibition of a single target by switching to an alternate mode for survival. However, by utilizing the endogenous response to a single insult (primary therapeutic), a second inhibitor may be used as a mixture with the primary therapeutic for a more effective treatment. Combinatorial approaches have previously been shown to improve various cancer therapies (Engelman et al., 2007; Fitzgerald et al., 2006; Ma et al., 2005; Namiki et al., 2006; Stommel et al., 2007; Toschi and Janne, 2008; Yasui et al., 2007). To this end, we combined a selective p38MAPK inhibitor, SB202190, with deguelin to suppress the endogenous p38MAPK response to deguelin alone (Figure 4.4). Additionally, when used as a therapeutic mixture, efficacy of deguelin was maintained (Figure 4.5). While SB202190 has been shown to be a selective p38MAPK inhibitor, we recognize that inhibitors are not perfect and may alter the activity of undesired intracellular proteins (Muniyappa and Das, 2008). Additionally, SB202190 has been shown to be toxic in a human leukemia cell line 24 hours post-exposure at higher doses $(50 \mu \mathrm{M})$ (Nemoto et al., 1998). In this study, we found that SB202190 (350 nM) alone did not significantly decrease HepG2 viability 24 hours post-exposure (Figure 4.5). It should be noted that the diverse role of p38MAPK signaling, for example the conflicting results during ischemia/reperfusion injury (for review, see Steenbergen, 2002), after cellular stress or xenobiotic exposure can vary based on the model system used (Cuadrado and Nebreda. 2010). 
By using this directed mixtures approach, the undesired alternative action of deguelin (inhibition of oxygen consumption) can be ameliorated without sacrificing efficacy. From Figure 4.6, SB202190 treatment alone decreased oxygen consumption slightly, but treatment with the various mixtures led to an increase in oxygen consumption when compared to deguelin alone, thus minimizing deguelin's undesired effect on oxygen consumption. Most notably, the highest mixture dose showed an increase in oxygen consumption when compared to $100 \mu \mathrm{M}$ of deguelin alone, while maintaining efficacy (less than 15\% viability). These results are consistent with previous studies that monitored mitochondrial bioenergetics in response to ETC inhibitors (Arvier et al., 2007; Dumas et al., 2003; Roussel et al., 2003). Desquiret and coworkers (2008) found that dexamethasone, a synthetic glucocorticoid, inhibits mitochondrial activity (specifically complex I and II) in HepG2 cells. Since glucocorticoid stimulation activates G protein-coupled receptor (GPCR), they hypothesized that GPCR mediated kinase pathways may be key players in regulating rapid glucocorticoid activity at ETC complexes. From this, they co-exposed HepG2 cells to dexamethasone and SB202190 (since p38MAPK is downstream of GPCR), and found that SB202190 increased Complex I activity by $80 \%$, when compared to HepG2 cells exposed to dexamethasone alone, which demonstrates the vital role p38MAPK plays in mediating mitochondrial bioenergetics after xenobiotic exposure. By using our proposed mixtures approach, the undesired alternative action of deguelin (inhibition of oxygen consumption) was diminished by inhibiting p38MAPK.

As a proof of principle, the novel mixtures approach presented here exploits the endogenous intracellular response to a primary therapeutic, revealing a secondary target 
for a combinatorial therapeutic regimen. By using this endogenously selected mixture, the cell's native response mechanism against a primary therapeutic is inhibited, making the intracellular signaling network less effective at cell recovery and decreasing viability. From this directed mixtures approach, a primary therapeutic's efficacy can be maintained, while potentially decreasing undesired side effects. Additionally, by using this approach, previously unsuccessful therapeutics derived from toxins can be revisited. This ultimately speaks to the significance of pharmacodynamic mixtures that target kinase responses as an avenue of future research.

\subsection{References}

Agarwal, R., Deep, G., 2008. Kava, a tonic for relieving the irrational development of natural preventive agents. Cancer Prev. Res. 6, 409-412.

Aggarwal, B.B., Takada, Y., Oommen, O.V., 2004. From chemoprevention to chemotherapy: common targets and common goals. Expert Opin. Investig. Drugs, 13, 1327-1338.

Arvier, M., Lagoutte, L., Johnson, G., Dumas, J.F., Sion, B., Grizard, G., Malthièry, Y., Simard, G., Ritz, P., 2007. Adenine nucleotide translocator promotes oxidative phosphorylation and mild uncoupling in mitochondria after dexamethasone treatment. Am. J. Physiol. Endocrinol. Metab. 293, E1320-E1324.

Boyd, J., Vrana, J.A., Williams, H.N., 2012. In Vitro Approach to Predict PostTranslational Phosphorylation Response to Mixtures. Toxicology 313, 113-21.

Caboni, P., Sherer, T.B., Zhang, N., 2004. Rotenone, deguelin, their metabolites and the rat model of Parkinson's disease. Chem. Res. Toxicol. 17, 1540-1548.

Chang, L.S., Chou, Y.C., Lin, S.R., Wu, B.N., Lin, J., Hong, E., Sun, Y.J., Hsiao, C.D., 1997. A novel neurotoxin, cobrotoxin b, from Naja naja atra (Taiwan cobra) venom: purification, characterization, and gene organization. J. Biochem. 122, 1252-1259.

Chun, K.H., Kosmeder II, J.W., Sun, S., Pezzuto, J.M., Lotan, R., Hong, W.K., Lee, H.Y., 2003. Effects of deguelin on the phosphatidylinositol 3-kinase/Akt pathway 
and apoptosis in premalignant human bronchial epithelial cells. J. Natl. Cancer Inst. 95, 291-302.

Cohen, P., 2002. Protein kinases- the major drug targets of the twenty-first century? Nat. Rev. Drug Discov. 1, 309-315.

Collins, I., Workman, P., 2006. New approaches to molecular cancer therapeutics. Nat. Chem. Biol. 2, 689-700.

Cuadrado, A., Nebreda, A.R., 2010. Mechanisms and functions of p38 MAPK signalling. Biochem. J. 429, 403-417.

Dancey, J., Sausville, E.A., 2003. Issues and progress with protein kinase inhibitors for cancer treatment. Nat. Rev. Drug Discov. 2, 296-313.

Desquiret, V., Gueguen, N., Malthièry, Y., Ritz, P., Simard, G., 2008. Mitochondrial effects of dexamethasone imply both membrane and cytosolic-initiated pathways in HepG2 cells. Int. J. Biochem. Cell Biol. 40, 1629-1641.

Dolcet, X., Llobet, D., Pallares, J., Matias-Guiu, X., 2005. NF-kB in development and progression of human cancer. Virchows Arch. 446, 475-482.

Dong, C., Davis, R.J., Flavell, R.A., 2002. MAP Kinases in the immune response. Annu. Rev. Immunol. 20, 55-72.

Dumas, J.F., Simard, G., Roussel, D., Douay, O., Foussard, F., Malthiery, Y., Ritz, P., 2003. Mitochondrial energy metabolism in a model of undernutrition induced by dexamethasone. Br. J. Nutr. 90, 969-977.

Engelman, J.A., Zejnullahu, K., Mitsudomi, T., Song, Y., Hyland, C., Park, J.O., Lindeman, N., Gale, C.-M., Zhao, X., Christensen, J., Kosaka, T., Holmes, A., Rogers, A.M., Capuzzo, F., Mok, T., Lee, C., Johnson, B.E., Cantley, L.C., Janne, P.A., 2007.MET amplification leads to gefitinib resistance in lung cancer by activating ERBB3 signaling. Science 316, 1039-1043.

Fang, N., Casida, J.E., 1998. Anticancer action of cubé insecticide: Correlation for rotenoid constituents between inhibition of NADH:ubiquinone oxidoreductase and induced ornithine decarboxylase activities. Proc. Natl. Acad. Sci. USA. 95, $3380-3384$.

Ferreira, S.H., 1965. A bradykinin-potentiating factor (bpf) present in the venom of bothrops jararaca. Br. J. Pharmacol. Chemother. 24, 163-169. 
Fitzgerald, J.B., Schoeberl, B., Nielsen, U.B., Sorger, P.K., 2006. Systems biology and combination therapy in the quest for clinical efficacy. Nat. Chem. Biol. 2, 458466.

Gerhauser, C., Lee, S.K., Kosmeder, J.W., Moriarty, R.M., Hamel, E., Mehta, R.G., Moon, R.C.,Pezzuto, J.M., 1997. Regulation of ornithine decarboxylase induction by deguelin, a natural product cancer chemopreventive agent.Cancer Res. 57, 3429-3435.

Jackson, R. C., 1993. Amphibolic drug combinations: the design of selective antimetaboliteprotocols based upon the kinetic properties of multienzyme systems. Cancer Res. 53, 3998-4003.

Jin, Z., El-Deiry, W.S., 2005. Overview of cell death signaling pathways. Cancer Biol. Ther. 4, 139-163.

Jones, P.H., Christodoulos, K., Dobbs, N., Thavasu, P., Balkwill, F., Blann, A.D., Caine, G.J., Kumar, S., Kakkar, A.J., Gompertz, N., Talbot, D.C., Ganesan, T.S., Harris, A.L., 2004. Combination antiangiogenesis therapy with marimastat, captopril and fragmin in patients with advanced cancer. Br. J. Cancer 91, 30-36.

Kholodenko, B.N., 2006. Cell signaling dynamics in time and space. Nat. Rev. Mol. Cell. Biol. 7, 165-176.

Kim, W.Y., Chang, D.J., Hennessy, B., Kang, H.J., Yoo, J., Han, S.H., Kim, Y.S., Park, H.J., Seo, S.Y., Mills, G., Kim, K.W., Hong, W.K., Suh, Y.G., Lee, H.Y., 2008. A novel derivative of the natural agent deguelin for cancer chemoprevention and therapy. Cancer Prev. Res. 7, 577-587.

Knasmuller, S., Parzefall, W., Sanyal, R., Ecker, S., Schwab, C., Uhl, M., 1998. Use of metabolically competent human hepatoma cells for the detection of mutagens and antimutagens. Mutat. Res. 402, 185-202.

Koh, C. Y., Kini, R. M., 2012. From snake venom toxins to therapeutics - cardiovascular examples. Toxicon 59, 497-506.

Lee, H.Y., 2004. Molecular mechanisms of deguelin-induced apoptosis in transformed human bronchial epithelial cells. Biochem. Pharmacol. 68, 1119-1124.

Lee, H.Y., Oh, S.H., Woo, J.K., 2005. Chemopreventive effects of deguelin, a novel Akt inhibitor, on tobacco-induced lung tumorigenesis. J. Natl. Cancer Inst. 97, 16951699.

Lee, J.C., Laydon, J.T., McDonnell, P.C., Gallagher, T.F., Kumar, S., Green, D., McNulty, D., Blumenthal, M.J., Keys, J.R., Landvatter, S.W., Strickler, J.E., 
McLaughlin, M.M., Siemens, I.R., Fisher, S.M., Livi, G.P., White, J.R., Adams, J.L., Young, P.R., 1994. A protein kinase involved in the regulation of inflammatory cytokine biosynthesis. Nature 372, 739-746.

Ma, P.C., Schaefer, E., Christensen, J.G., Salgia, R., 2005. A selective small molecule cMET inhibitor, PHA665752, cooperates with rapamycin. Clin. Cancer Res. 11, 2312-2319.

Mersch-Sundermann, V., Knasmuller, S., Wu, X.J., Darroudi, F., Kassie, F., 2004. Use of a human-derived liver cell line for the detection of cytoprotective, antigenotoxic and cogenotoxic agents. Toxicology 198, 329-340.

McCleary, R.J.R., Kini, R.M., 2012. Non-enzymatic proteins from snake venoms: A gold mine of pharmacological tools and drug leads. Toxicon http://dx.doi.org/10.1016/j.toxicon.2012.09.008

Muniyappa, H., Das, K.C., 2008. Activation of c-Jun N-terminal kinase (JNK) by widely used specific p38 MAPK inhibitors SB202190 and SB203580: a MLK-3-MKK7dependent mechanism. Cell Signal. 20, 675-683.

Murillo, G., Salti, G.I., Kosmeder II, J.W., Pezzuto, J.M., Mehta, R.G., 2002. Deguelin inhibits the growth of colon cancer cells through the induction of apoptosis and cell cycle arrest. Eur. J. Cancer 38, 2446-2454.

Namiki, Y., Namiki, T., Yoshida, H., Date, M., Yashiro, M., Matsumoto, K., Nakamura, T., Yanagihara, K., Tada, N., Satoi, J., Fujise, K., 2006. Preclinical study of a "tailor-made" combination of NK4-expressing gene therapy and gefitinib (ZD1839, Iressa) for disseminated peritoneal scirrhous gastric cancer. Int. J. Cancer 118, 1545-1555.

Nemoto, S., Xiang, J., Huang, S., Lin, A., 1998. Induction of apoptosis by SB202190 through inhibition of p38beta mitogen-activated protein kinase. J. Biol. Chem. 273, 16415-16420.

Neuwinger, H.D., 2004. Plants used for poison fishing in tropical Africa. Toxicon 44, 417-430.

Oh, S.H., Woo, J.K., Yazici, Y.D.,Myers, J.N., Kim, W.Y., Jin, Q., Hong, S.S., Park, H.J., Suh, Y.G., Kim, K.W., Hong, W.K., Lee, H.Y.,2007. Structural Basis for Depletion of Heat Shock Protein 90 Client Proteins by Deguelin. J. Natl. Cancer Inst. 99, 949-961.

Oh, S.H., Woo, J.K.,Jin, Q., Kang, H.J., Jeong, J.W., Kim, K.W., Hong, W.K., Lee, H.Y., 2008. Identification of novel antiangiogenic anticancer activities of deguelin targeting hypoxia-inducible factor-1 $\alpha$. Int. J. Cancer. 122, 5-14. 
Oren, M., 2003. Decision making by p53: life, death and cancer. Cell Death Differ. 10, 431-442.

Park, M.H., Song, H.S., Kim, K.H., Son, D.J., Lee, S.H., Yoon, D.Y., Kim, Y., Park, I.Y., Song, S., Hwang, B.Y., Jung, J.K., Hong, J.T., 2005. Cobrotoxin inhibits NFkappa B activation and target gene expression through reaction with NF-kappa B signal molecules. Biochemistry 44, 8326-8336.

Peng, X.H., Karna, P., O'Regan, R.M., 2007. Down-regulation of inhibitor of apoptosis proteins by deguelin selectively induces apoptosis in breast cancer cells. Mol. Pharmacol. 71, 101-11.

Roussel, D., Dumas, J.F., Augeraud, A., Douay, O., Foussard, F., Malthiéry, Y., Simard, G., Ritz, P., 2003. Dexamethasone treatment specifically increases the basal proton conductance of rat liver mitochondria. FEBS Lett. 541, 75-79.

Sauro, H.M., Kholodenko, B.N., 2004. Quantitative analysis of signaling networks. Prog. Biophys. Mol. Biol. 86, 5-43.

Steenbergen, C., 2002. The role of p38 mitogen-activated protein kinase in myocardial ischemia/reperfusion injury; relationship to ischemic preconditioning. Basic Res. Cardiol. 97, 276-285.

Stommel, J.M., Kimmelman, A.C., Ying, H., Nabioullin, R., Ponugoti, A.H., Wiedemeyer, R., Stegh, A.H., Bradner, J.E., Ligon, K.L., Brennan, C., Chin, L.,DePinho, R.A., 2007. Coactivation of receptor tyrosine kinases affects the response of tumor cells to targeted therapies. Science 318, 287-290.

Toschi, L., Janne, P.A., 2008. Single-agent and combination therapeutic strategies to inhibit hepatocyte growth factor/MET signaling in cancer. Clin. Cancer Res. 14, 5941-5946.

Udeani, G.O., Gerhauser, C., Thomas, C.F., Moon, R.C., Kosmeder, J.W., Kinghorn, A.D., Moriarty, R.M., Pezzuto, J.M., 1997. Cancer chemopreventive activity mediated by deguelin, a naturally occurring rotenoid. Cancer Res. 57, 3424-3428.

Vander Heiden, M.G., Cantley, L.C.,Thompson, C.B., 2009. Understanding the Warburg effect: the metabolic requirements of cell proliferation. Science 324, 1029-1033.

Warburg, O., 1956. On the origin of cancer cells. Science 123, 309-314.

Wilkinson, M.G., Millar, J.B.A., 2000. Control of the eukaryotic cell cycle by MAP kinase signaling pathways. FASEB J. 14, 2147-2157. 
Yasui, H., Hideshima, T., Ikeda, H., Jin, J., Ocio, E.M., Kiziltepe, T., Okawa, Y., Vallet, S., Podar, K., Ishitsuka, K., Richardson, P.G., Pargellis, C., Moss, N., Raje, N.,Anderson, K.C., 2007. BIRB 796 enhances cytotoxicity triggered by bortezomib, heat shock protein (Hsp) 90 inhibitor, and dexamethasone via inhibition of p38 mitogen-activated protein kinase/Hsp27 pathway in multiple myeloma cell lines and inhibits paracrine tumour growth.Br. J. Haematol. 136, 414-423. 


\section{Chapter 5}

Future directions: Expanding mixtures concepts to human interaction 


\section{Future directions: Expanding concepts to human interaction}

The work presented in this dissertation was meant to understand early cellular changes, whether that be alterations in cellular bioenergetics or pathway perturbations, and use the resulting mechanistic information to formulate new toxicity prediction models. With better prediction models that harness the mechanistic information from well planned, high-throughput in vitro experiments, many more individual chemicals and mixtures can be properly assessed for toxicity risk assessment. The success of the prediction models presented in this dissertation may greatly impact xenobiotic highthroughput mixtures risk assessment in vitro, as well as pharmaceutical adverse drug reaction analyses. Additionally, this fundamental research may be expanded to human research interaction studies, where intercommunication and team dynamics may contribute to responses of individuals on a team (human mixtures). Preliminary research regarding this new application is presented in this chapter.

\subsection{Introduction}

Perturbations of biological processes by chemical stress exposure can elicit early cellular changes, leading to an adaptive stress response for continued survival or adverse response leading to toxicity (Andersen, 2010). The term "stress" is not exclusive to exogenous xenobiotics, but also extends to external stimuli that initiate endogenous stress signaling biomolecules, such as cytokines, hormones, and catecholamines, to name a few (Boelsterli, 2007). In light of this classification, the same high-throughput approach for measuring xenobiotic chemical stress for individual compounds or chemical mixtures 
may be applied to human interaction studies to understand the dynamic molecular response to social stress and teamwork. With this approach, teams can be constructed to reduce individual "stress," improve teamwork, and develop models incorporating human interaction stress responses for risk assessment.

In the presence of stress-inducing stimuli, there is bimodal communication between the brain and body, which is coordinated by the autonomic nervous system (ANS), endocrine and immune systems (McEwen, 2005; McEwen, 2007). This bimodal communication relies on dynamic chemical messengers released to promote adaptation (allostasis) in response to environmental, psychological, and physiological stressors (Chida and Hamer, 2008). The initiation of these messengers can be measured as early as seconds to minutes after an individual perceives a situation or stimuli as a threat (Sapolsky et al., 2000). The perception of threat and allostatic responses to external stimuli, whether they are real or imagined, can vary depending upon individual inherent (genetic makeup or development), behavioral (lifestyle choices and mechanisms for coping), and historical (previous trauma or major life events) factors (McEwen, 1998; Juster et al., 2010). These components drive individual resiliency or sensitivity to stress. Sensitivity to repeated acute stressors over time can lead to chronic stress and disease states. The Allostatic Load (AL) model has been used over the last two decades to better understand the physiology of stress, especially as it relates to effects of stress in the workplace and at home (McEwen and Stellar, 1993; Ganster and Rosen, 2013). The AL model has numerous potential applications for various fields of study to monitor individuals and teams that are under substantial amounts of stress, whether it be medical (doctors, nurses, paramedics), business and management (market traders, both small and large companies), athletic teams, or military units. 
Research investigating social relationships as they relate to physical health has been well documented (House et al., 1988; Umberson and Montez, 2010). Supportive relationships and interactions have been shown to improve immune, endocrine, and cardiovascular responses to stress and reduce overall AL (Cohen, 2004; Kiecolt-Glaser $e t$ al., 2005). With this in mind, allostatic responses to a perceived threat, competition, or risk taking may be different when a task is performed as a team rather than an individual.

In the presence of acute stress-inducing stimuli, the $\mathrm{AL}$ model proposes the following mechanism for primary processes for initial adaptation to stress. Primary processes of the AL model include psychological (fear or anxiety), physiological (cortisol, catecholamines, cytokines), and psychosomatic (fatigue or sleep disturbance) mediators (McEwen, 2003; Ganster and Rosen, 2013). Recent trends in AL research suggest moving away from single biomarkers to a more inclusive and comprehensive multiplex approach (Khan, 2012; Ganster and Rosen, 2013), casting a wide net on multiple physiological pathways (neuroendocrine, immune, metabolic, and cardiovascular) to elucidate an individual's acute and/or chronic stress response, similar to the recent NRC report suggestions (NRC, 2007).

To this end, we initiated a human research study to monitor individual and team physiological metrics and elucidate adaptive biomolecular responses relevant to teamwork stress and performance. In this study, we enrolled Air Force Reserve Officers' Training Corps (ROTC) cadets to perform a mock hostage rescue mission alone and as part of a four person team. During the study, we measured an array of salivary biomarkers related to the various pathways of an individual's stress response for potential elucidation of the major and minor physiological mediators driving adaptive stress 
responses while on a team. We hypothesized that individual salivary responses would increase as part of a team, due to increased demands and forced social interaction to complete a task. This work opens the door for a new avenue of mixtures research, where interaction with other individuals in combination with emotional, physical, or chemical exposure, can be considered as a component that may alter an individual's response.

\subsection{Materials and Methods}

\subsubsection{Subjects}

The present study enrolled 17 subjects (18-22 y of age) from West Virginia University's Air Force Reserve Officers' Training Corps (ROTC). Subjects were introduced to the study during a university ROTC course, where the background rationale, goals and risks were described in a power point presentation. Those in attendance who were interested in participating were asked to provide their email address to contact about availability for the study and to go over consent. A total of 17 subjects were originally enrolled in the study (16 male, 1 female). After the individual phase of the study, where subjects performed the tasks alone, one subject was dropped from the study due to sampling errors. A total of 16 subjects (15 male, 1 female) were used for the team portion of the study. Subjects were placed on teams using a random number generator and their schedule availability. This study received approval from the Institutional Review Board committee at West Virginia University and the United States Army Medical Research and Materiel Command (USAMRMC) Office of Research Protections. 


\subsubsection{Salivary biomarkers}

Saliva samples were collected immediately (less than 5 minutes) before the mission clock started. Samples were collected with saliva collection aids purchased from Salimetrics (State College, PA). All subjects had refrained from eating at least one hour prior to sample collection. Unstimulated saliva samples were collected using the passive drool method. Saliva samples were stored on ice (less than $3 \mathrm{~h}$ ) before centrifugation (to remove saliva debris) and long-term storage at $-80^{\circ} \mathrm{C}$. Salivary cytokines were assayed using commercial kits. Salivary cytokines (GM-CSF, IFN- $\gamma$, IL-1 $\beta$, IL-2, IL-4, IL-5, IL6, IL-8, IL-10 and TNF- $\alpha$ ), were assayed using a commercial magnetic bead-based suspension array 10-plex cytokine kit (cat. no. LHC0001M) purchased from Life Technologies (Carlsbad, CA). Assay was performed in triplicate $(25 \mu \mathrm{L} / a s s a y$ well $)$ according to manufacturer's protocol and measured with a Bio-plex 200 platform (BioRad, Hercules, CA). All samples (individual and team) analyzed with cytokine kits were assayed over the course of a week.

\subsection{Results and Discussion}

The human body, especially endocrine, immune, and cardiovascular system, is exceedingly sensitive and responsive to social interactions (Heaphy and Dutton, 2008). These social interactions, whether they are with peers or those higher/lower in employee/team status, can have a substantial impact on workplace efficiency, performance, and, potentially, workplace-induced stress. As a key player in immune surveillance and response, the mouth is in constant contact with airborne pollutants that 
are bacterial, viral, or chemical in nature (O'Connor et al., 2009). To gain a better understanding of team dynamics and the impact of teamwork on an individual, we compared initial salivary cytokine responses when subjects performed our mock hostage rescue mission as an individual to initial salivary cytokine responses when they performed it as part of a team. As an individual, subjects' initial summed salivary cytokine responses (IL-1 $\beta$, IL-10, IL-6, GM-CSF, IL-5, IFN- $\gamma$, TNF- $\alpha$, IL-2, and IL-4) were lower than when performing the mission as part of a four person team (Figures 5.12 ), with the exception of subject 4 ; thus agreeing with our hypothesis that the team scenario would induce higher initial salivary cytokine levels than when they arrived to perform the mission as an individual.
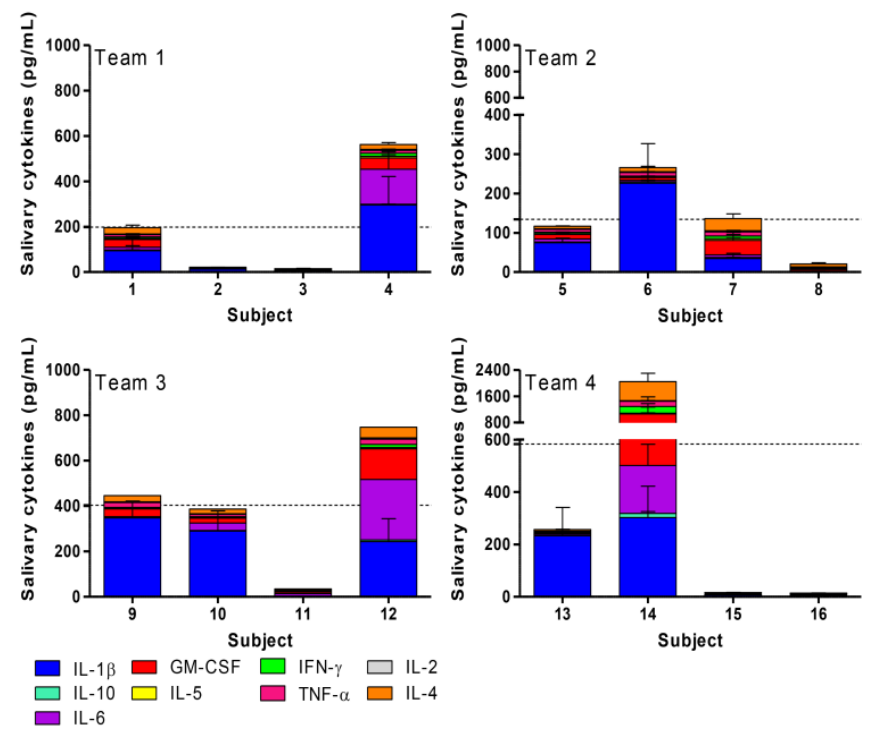

Figure 5.1 Initial salivary cytokine responses as an individual. Salivary samples were collected via passive drool method less than 5 minutes before the first mission started for the individual portion of the study. Samples were assayed using the multiplex bead-based ELISA. Horizontal line represents mean for the 4 individuals on their eventual team (organized by team to easier visualization). Error bars represent S.E.M.

Additionally, subjects' summed salivary cytokine levels were significantly different when comparing individual and team responses $(P<0.01$, paired student's $\mathrm{t}-$ test). However, in relation to performance, the time to complete the mock hostage rescue mission as an individual (Table 5.1) was not significantly correlated to any single or 

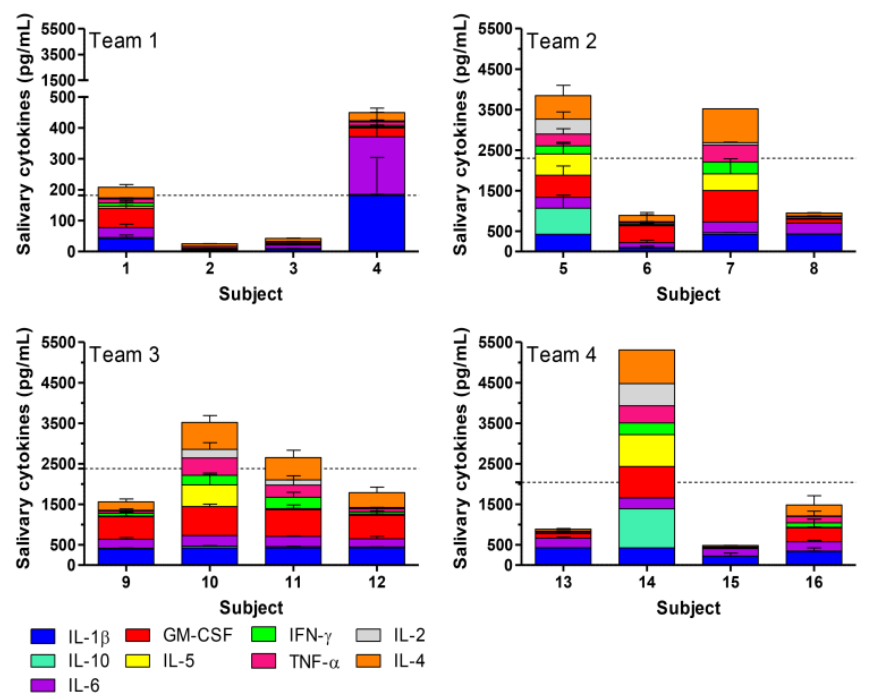

Figure 5.2 Initial salivary cytokine responses as a team. Salivary samples were collected via passive drool method less than $5 \mathrm{~min}$ before the first mission of the team portion of the study. Samples were assayed using the multiplex bead-based ELISA. Horizontal line represents mean for the 4 individuals on a team (during the team portion of the study). Error bars represent S.E.M.

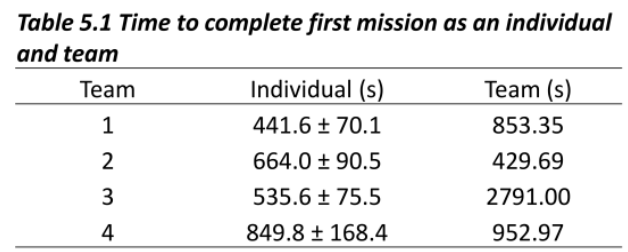

$\begin{aligned} & \text { Table 5.2. Mean summed cytokine responses as an } \\
& \text { individual and team }\end{aligned}$
\begin{tabular}{ccc}
\hline Team & Individual $(\mathrm{pg} / \mathrm{mL})$ & Team $(\mathrm{pg} / \mathrm{mL})$ \\
\hline 1 & $198.7 \pm 128.4$ & $181.6 \pm 98.5$ \\
2 & $134.6 \pm 50.4$ & $2304 \pm 800.4$ \\
3 & $403.9 \pm 146.2$ & $2382 \pm 446.3$ \\
4 & $582.9 \pm 490.8$ & $2042 \pm 1109$ \\
\hline
\end{tabular}

summed salivary cytokine response (Table 5.2). This relation was also true for initial salivary cytokine concentrations when performing the mission as part of a team.

The mechanism driving salivary cytokine responses after social interaction exposure has yet to be elucidated. The whole-organism signaling response (intra- and inter- cellular, as well as disparate organ system communication) involves a complex spatiotemporal coordination of multiple components, whether they be proteins or biomolecules, to integrate the stressful stimuli and produce a response. Neural-immune (e.g, brain-cytokine) communication between the immune system and the central nervous system (CNS) involves the coordination of many key players, such as the vagus, glossopharyngeal, and trigeminal nerves, HPA axis, SNS, peripheral nervous system 
(PNS), and their corresponding downstream targets (tissues and organs) (Romeo et al., 2001; Navarro et al., 2006; Thayer and Sternberg, 2010). The parasympathetic nervous system (both afferent and efferent activity) in particular has been shown to be a major player in neural-immune communication (Sternberg, 2006). It has been suggested that the vagus nerve is highly innervated with IL-1 receptors (Goehler et al., 1997), and the presence of afferent branches of vagus nerve (pharyngeal and laryngeal) in the mouth and pharynx, as well as the overall gustatory system (Berthoud, 2008), may act as sensory nerves for salivary IL-1 $\beta$ related to local immune responses or social and psychological stressors. In addition to the vagus nerve, immune to brain communication may be relayed by glossopharyngeal nerve afferents present in the posterior oral cavity (Watkins and Maier, 2005; Goehler, 2008). In a study relating the glossopharyngeal nerve and immune to brain communication, rats that underwent a bilateral glossopharyngeal nerve transection were exposed to either lipopolysaccharide (LPS) or IL-1 $\beta$ via injection into the soft palate (ISP) (Romeo et al., 2001). Sham rats exposed to LPS or IL-1 $\beta$ via ISP showed a febrile dose-response. However, rats with bilateral glossopharyngeal nerve transection had a reduced febrile response after LPS or IL-1 $\beta$ exposure, suggesting the critical role of the glossopharyngeal nerve for immune to brain communication from the oral cavity. The trigeminal nerve is also a key player in immune to brain communication. The trigeminal nerve provides afferent somatosensory innervation to the face and oral cavity in mammals (Byers and Dong, 1989). To investigate the relationship between acute oral inflammation and brain communication, Navarro and colleagues (Navarro et al., 2006) injected LPS into periodontal tissue and measured their febrile response. They found that local periodontal inflammation induced a febrile response and maxillary 
trigeminal nerve transection attenuated this febrile response, suggesting the trigeminal nerve is involved in oral inflammation to brain communication.

\subsection{Conclusion}

Military teams experience work environments that are both dynamic and complex, under potentially life threatening circumstances, which indicates a high workload (Cannon-Bowers et al., 1992; Urban et al., 1995). Even though these military units undergo advanced physical and mental training in preparation for future missions, performance may decline when it is most critical (i.e., during a mission) (Sandal et al., 1998). Selection of a team that will withstand and adapt to any external stressors and work as a cohesive unit toward mission success is imperative; both metrics and methods for selecting these individuals are needed. In this study, we have shown that for a mock hostage rescue mission in an urban setting, summed initial salivary cytokine responses increase when the mission was performed with teammates as opposed to individually, suggesting a relationship between potential social stress and salivary cytokines.

\subsection{References}

Andersen, M.E., 2010. Calling on science: making "alternatives" the new gold standard. ALTEX 27, 29-37.

Berthoud, H.R., 2008. The vagus nerve, food intake and obesity. Regul Pept 149, 15-25.

Boelsterli, U.A., 2007. Mechanistic Toxicology: The Molecular Basis of How Chemicals Disrupt Biological Targets, Second Edition. CRC Press, Boca Raton, FL. 
Byers, M.R., Dong, W.K., 1989. Comparison of trigeminal receptor location and structure in the periodontal ligament of different types of teeth from the rat, cat, and monkey. J Comp Neurol 279, 117-127.

Cannon-Bowers, J.A., Oser, R., Flanagan, D.L., 1992. Work teams in industry: A selected review and proposed framework. Ablex Publishing, Norwood, NJ.

Chida, Y., Hamer, M., 2008. Chronic psychosocial factors and acute physiological responses to laboratory-induced stress in healthy populations: a quantitative review of 30 years of investigations. Psychol Bull 134, 829-885.

Cohen, S., 2004. Social relationships and health, Am Psychol, United States, pp. 676684.

Ganster, D.C., Rosen, C.C., 2013. Work stress and employee health: A multidisciplinary review. Journal of Management 39, 1085-1122.

Goehler, L.E., 2008. Cytokines in Neural Signaling to the Brain. 6, 337-352.

Goehler, L.E., Relton, J.K., Dripps, D., Kiechle, R., Tartaglia, N., Maier, S.F., Watkins, L.R., 1997. Vagal paraganglia bind biotinylated interleukin-1 receptor antagonist: a possible mechanism for immune-to-brain communication. Brain Res Bull 43, 357-364.

Heaphy, E.D., Dutton, J.E., 2008. Positive Social Interactions and the Human Body at Work: Liking Organizations and Physiolgy. Academy of Management Review 33, 137-162.

House, J.S., Landis, K.R., Umberson, D., 1988. Social relationships and health. Science 241, 540-545.

Juster, R.P., McEwen, B.S., Lupien, S.J., 2010. Allostatic load biomarkers of chronic stress and impact on health and cognition. Neurosci Biobehav Rev 35, 2-16.

Khan, A., 2012. Detection and quantitation of forty eight cytokines, chemokines, growth factors and nine acute phase proteins in healthy human plasma, saliva and urine. $\mathbf{J}$ Proteomics 75, 4802-4819.

Kiecolt-Glaser, J.K., Loving, T.J., Stowell, J.R., Malarkey, W.B., Lemeshow, S., Dickinson, S.L., Glaser, R., 2005. Hostile marital interactions, proinflammatory cytokine production, and wound healing. Arch Gen Psychiatry 62, 1377-1384.

McEwen, B.S., 1998. Stress, adaptation, and disease. Allostasis and allostatic load. Ann N Y Acad Sci 840, 33-44.

McEwen, B.S., 2003. Interacting mediators of allostasis and allostatic load: towards an understanding of resilience in aging. Metabolism 52, 10-16. 
McEwen, B.S., 2005. Stressed or stressed out: what is the difference? J Psychiatry Neurosci 30, 315-318.

McEwen, B.S., 2007. Physiology and neurobiology of stress and adaptation: central role of the brain. Physiol Rev 87, 873-904.

McEwen, B.S., Stellar, E., 1993. Stress and the individual. Mechanisms leading to disease. Arch Intern Med 153, 2093-2101.

Navarro, V.P., Iyomasa, M.M., Leite-Panissi, C.R., Almeida, M.C., Branco, L.G., 2006. New role of the trigeminal nerve as a neuronal pathway signaling brain in acute periodontitis: participation of local prostaglandins. Pflugers Arch 453, 73-82.

NRC, 2007. Toxicity Testing in the 21st Century: A Vision and a Strategy. National Academies Press, Washington, D.C.

O'Connor, M.F., Irwin, M.R., Wellisch, D.K., 2009. When grief heats up: proinflammatory cytokines predict regional brain activation. Neuroimage 47, 891896.

Romeo, H.E., Tio, D.L., Rahman, S.U., Chiappelli, F., Taylor, A.N., 2001. The glossopharyngeal nerve as a novel pathway in immune-to-brain communication: relevance to neuroimmune surveillance of the oral cavity. J Neuroimmunol 115, 91-100.

Sandal, G.M., Gronningsaeter, H., Eriksen, H.R., Gravraakmo, A., Birkeland, K., Ursin, H., 1998. Personality and endocrine activation in military stress situations. Military Psychology 10, 45-61.

Sapolsky, R.M., Romero, L.M., Munck, A.U., 2000. How do glucocorticoids influence stress responses? Integrating permissive, suppressive, stimulatory, and preparative actions. Endocr Rev 21, 55-89.

Sternberg, E.M., 2006. Neural regulation of innate immunity: a coordinated nonspecific host response to pathogens. Nat Rev Immunol 6, 318-328.

Thayer, J.F., Sternberg, E.M., 2010. Neural aspects of immunomodulation: focus on the vagus nerve. Brain Behav Immun 24, 1223-1228.

Umberson, D., Montez, J.K., 2010. Social relationships and health: a flashpoint for health policy. J Health Soc Behav 51 Suppl, S54-66.

Urban, J.M., Bowers, C.A., Monday, S.D., Morgan Jr., B.B., 1995. Workload, team structure, and communication in team performance. Military Psychology 7, 123139. 
Watkins, L.R., Maier, S.F., 2005. Immune regulation of central nervous system functions: from sickness responses to pathological pain. J Intern Med 257, 139-155. 